Aus der Abteilung Hals-Nasen-Ohrenheilkunde

(Prof. Dr. med. Ch. Matthias)

im Zentrum Augenheilkunde und Hals-Nasen-Ohrenheilkunde

der Medizinischen Fakultät der Universität Göttingen

Retrospektive Analyse über die Therapie

mit Botulinumtoxin bei verschiedenen Dysphagieformen am Beispiel des oberen und unteren Ösophagussphinkters

\author{
Inaugural - Dissertation \\ zur Erlangung des Doktorgrades \\ der Medizinischen Fakultät \\ der Georg-August-Universität zu Göttingen \\ vorgelegt von \\ Katharina Bartsch \\ aus \\ Villingen-Schwenningen
}

Göttingen 2009 
De k a n:

I. Berichterstatter:

II. Berichterstatter/in:

III. Berichterstatter/in:

Tag der mündlichen Prüfung:
Prof. Dr. med. C. Frömmel

Prof. Dr. med. R. Laskawi 
I INHALTSVERZEICHNIS

II ABKÜRZUNGEN, ABBILDUNGEN UND TABELLEN ................................... IV

II.1 Liste der verwendeten Abkürzungen...................................................... IV

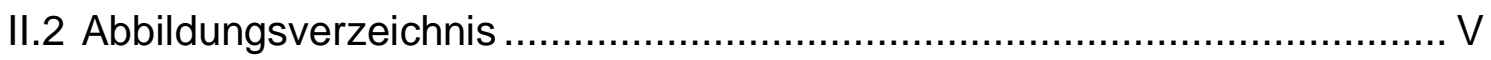

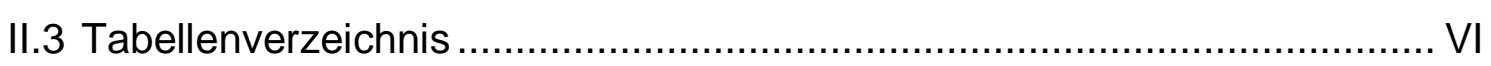

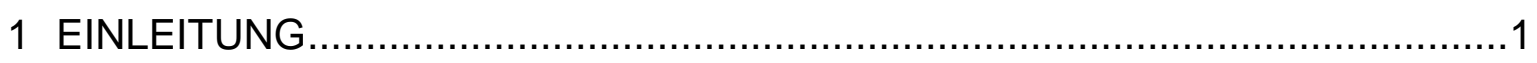

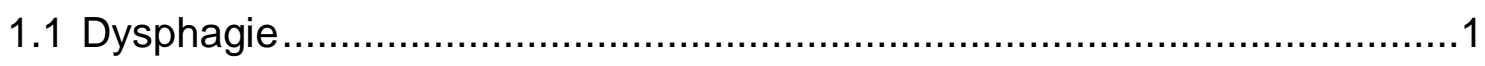

1.1.1 Das Symptom Dysphagie und der physiologische Schluckakt ............1

1.1.2 Unterteilung verschiedener Dysphagieformen aufgrund ihrer

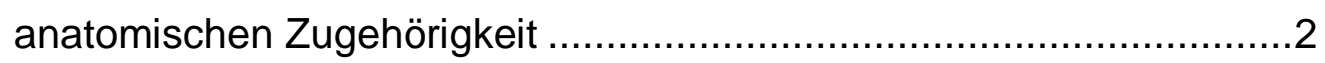

1.1.3 Oberer Ösophagussphinkter (UES) ..............................................

1.1.3.1 Anatomie des Ösophagus und des UES .............................

1.1.3.2 Pathophysiologie und Klinik der

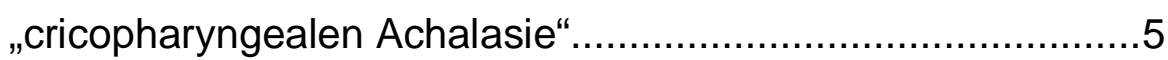

1.1.3.3 Diagnostik der pharyngealen Dysphagie ...............................6

1.1.4 Unterer Ösophagussphinkter (LES) .............................................

1.1.4.1 Anatomie und Physiologie des LES .....................................

1.1.4.2 Pathophysiologie und Klinik der Achalasie ............................9

1.1.4.3 Diagnostik der Achalasie ...................................................11

1.2 Konservative und operative Therapieverfahren bei Dysphagie..................14

1.2.1 Behandlungsmöglichkeiten des UES (oberer Ösophagussphinkter)...14

1.2.2 Behandlungsmöglichkeiten des LES (unterer Ösophagussphinkter) 15

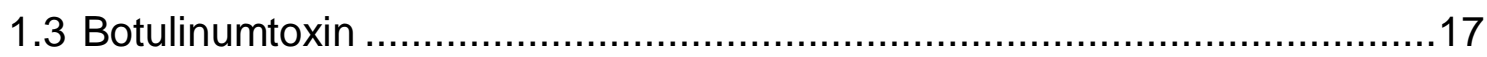

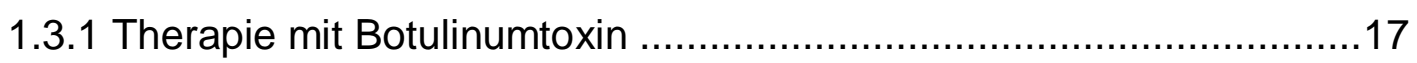

1.3.2 Wirkungsmechanismus der Botulinumtoxine .................................18

1.3.3 Regeneration der präsynaptischen Nervenzelle ..............................20

1.3.4 Messung der biologischen Aktivität von Botulinumtoxin....................21

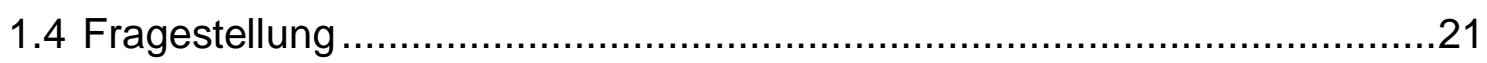

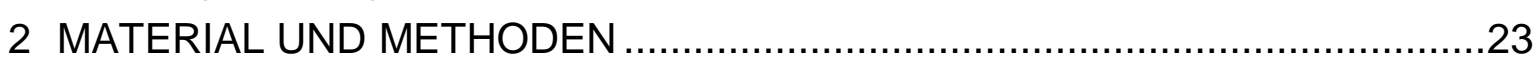

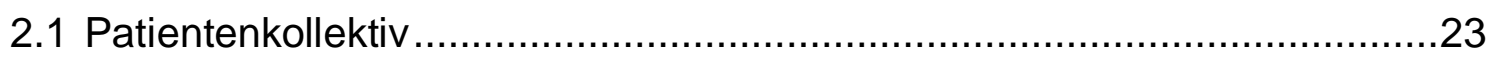

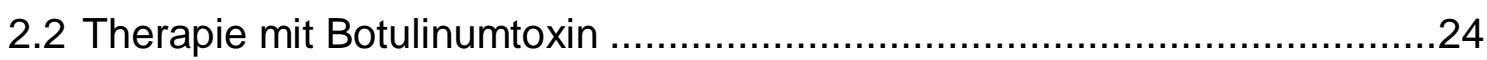

2.2.1 Behandlung im Bereich des UES (oberer Ösophagussphinkter) .......24

2.2.2 Behandlung im Bereich des LES (unterer Ösophagussphinkter).......26

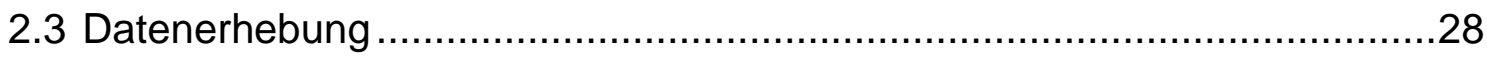

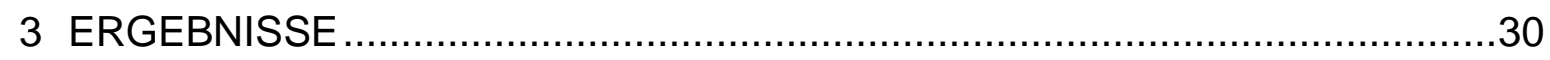

3.1 Charakterisierung des Patientenkollektivs ….........................................30

3.1.1 Gruppe UES (oberer Ösophagussphinkter) ...................................30 
3.1.2 Gruppe LES (unterer Ösophagussphinkter)....................................31

3.2 Begleiterkrankungen und Medikation des Patientenkollektivs ....................31

3.2.1 Begleiterkrankungen des Patientenkollektivs....................................31

3.2.1.1 Gruppe UES (oberer Ösophagussphinkter) .........................32

3.2.1.2 Gruppe LES (unterer Ösophagussphinkter).........................33

3.2.2 Medikation des Patientenkollektivs ................................................33

3.2.2.1 Gruppe UES (oberer Ösophagussphinkter) ..........................34

3.2.2.2 Gruppe LES (unterer Ösophagussphinkter).........................35

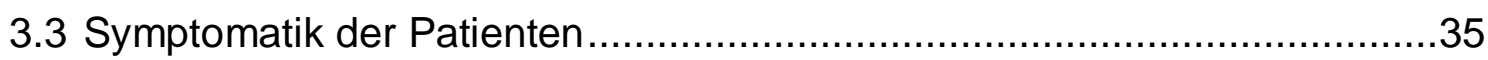

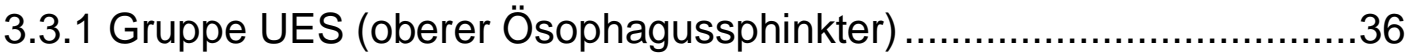

3.3.2 Gruppe LES (unterer Ösophagussphinkter) ...................................37

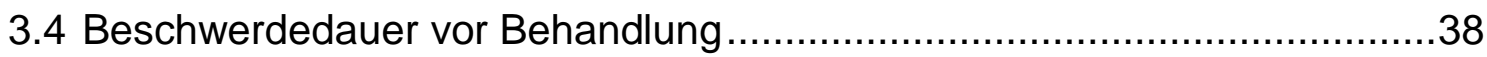

3.4.1 Gruppe UES (oberer Ösophagussphinkter) …................................39

3.4.2 Gruppe LES (unterer Ösophagussphinkter) ...................................39

3.5 Therapien vor Behandlung mit Botulinumtoxin A in der Universitätsklinik

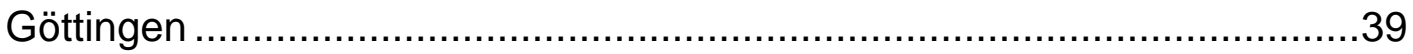

3.5.1 Gruppe UES (oberer Ösophagussphinkter) ................................. 40

3.5.2 Gruppe LES (unterer Ösophagussphinkter) ...................................40

3.6 Injektionen und Dosierungen von Botulinumtoxin A.................................41

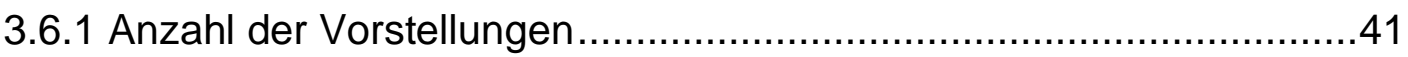

3.6.1.1 Gruppe UES (oberer Ösophagussphinkter) .........................41

3.6.1.2 Gruppe LES (unterer Ösophagussphinkter).........................42

3.6.2 Dosierungen von Botulinumtoxin A ............................................42

3.6.2.1 Gruppe UES (oberer Ösophagussphinkter) .........................43

3.6.2.2 Gruppe LES (unterer Ösophagussphinkter)..........................43

3.6.3 Zeitraum zwischen den Injektionen................................................

3.6.3.1 Gruppe UES (oberer Ösophagussphinkter) .........................44

3.6.3.2 Gruppe LES (unterer Ösophagussphinkter)........................45

3.6.4 Dosismodifikation und Intervallveränderungen im Verlauf bei

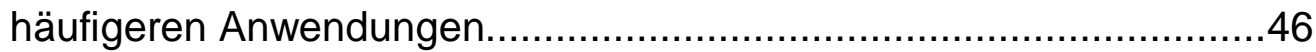

3.6.4.1 Gruppe UES (oberer Ösophagussphinkter) .........................46

3.6.4.2 Gruppe LES (unterer Ösophagussphinkter)........................47

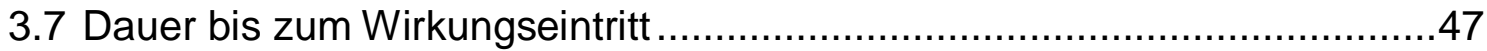

3.7.1 Gruppe UES (oberer Ösophagussphinkter) ...................................... 48

3.7.2 Gruppe LES (unterer Ösophagussphinkter) ..................................49

3.8 Vergleich des Therapieerfolgs von subjektiven Aussagen und objektiver

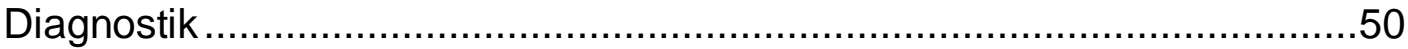

3.8.1 Gruppe UES (oberer Ösophagussphinkter) ......................................

3.8.2 Gruppe LES (unterer Ösophagussphinkter).....................................51 
3.9 Unerwünschte Wirkungen von Botulinumtoxin A ……..........................52

3.9.1 Gruppe UES (oberer Ösophagussphinkter) .................................52

3.9.2 Gruppe LES (unterer Ösophagussphinkter)..................................52

3.10 Vergleich des Patientenkollektivs anhand von Respondern und Non-

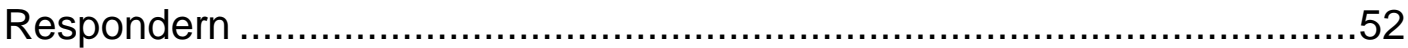

3.10.1 Gruppe UES (oberer Ösophagussphinkter) ……........................54

3.10.2 Gruppe LES (unterer Ösophagussphinkter)......................................

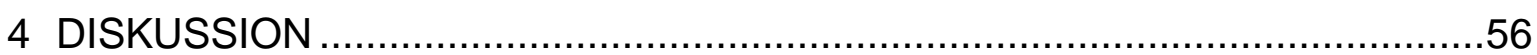

4.1 Wirkung von Botulinumtoxin A auf die Dysphagie ..................................56

4.1.1 Dysphagien mit Störungen im Bereich des UES (oberer Ösophagussphinkter).......................................................56

4.1.2 Dysphagien mit Störungen im Bereich des LES

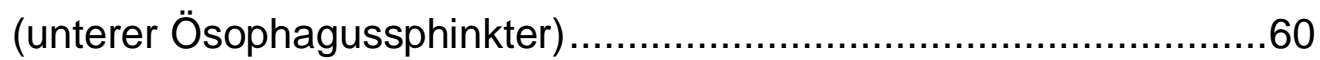

4.2 Vergleich von Respondern und Non-Respondern ...................................62

4.2.1 Gruppe UES (oberer Ösophagussphinkter) .....................................62

4.2.2 Gruppe LES (unterer Ösophagussphinkter) ....................................63

4.3 Vergleich von subjektiven und objektiven Ergebnissen ............................64

4.3.1 Gruppe UES (oberer Ösophagussphinkter) ...................................64

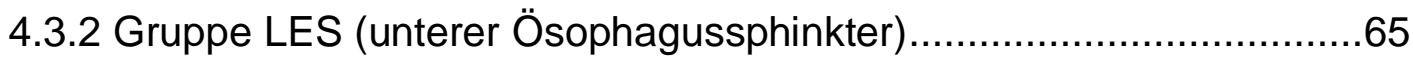

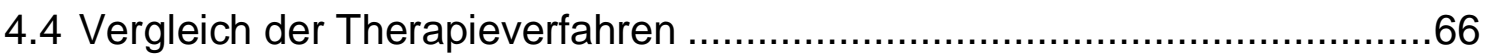

4.4.1 Gruppe UES (oberer Ösophagussphinkter) ..................................66

4.4.1.1 Ansprechrate und Wirkungsdauer ......................................66

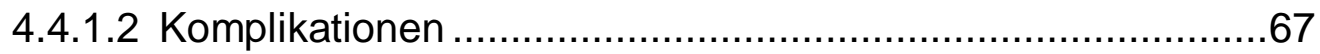

4.4.1.3 Botulinumtoxininjektion als Diagnostikum ............................68

4.4.2 Gruppe LES (unterer Ösophagussphinkter)...................................69

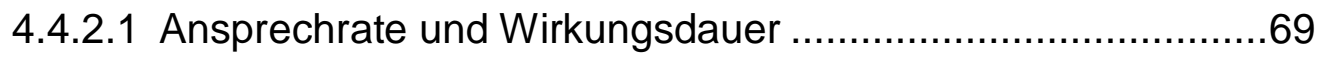

4.4.2.2 Komplikationen und Risiken..............................................70

4.4.2.3 Kombination von verschiedenen Therapien ..........................71

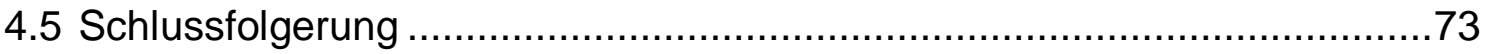

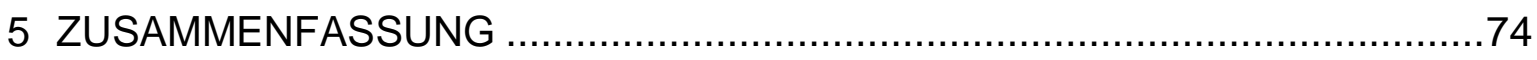

6 ANHANG: Einverständniserklärung und Behandlungsbogen ...........................75

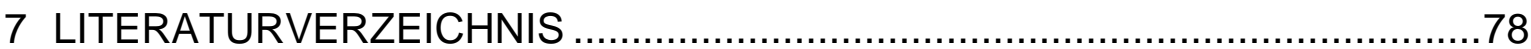




\section{Abkürzungen, Abbildungen und Tabellen}

\section{II.1 Liste der verwendeten Abkürzungen}

Abb.

Abbildung

ACh

Acetylcholin

bzw.

beziehungsweise

$\mathrm{cm}$

Zentimeter

COLD

chronisch-obstruktive Lungenerkrankung

CT

Computertomographie

EKG

Elektrokardiogramm

GIP

gastric inhibitory polypeptide

HMG-CoA

3-Hydroxy-3-methylglutaryl-CoA

$\mathrm{KHK}$

koronare Herzkrankheit

LES

Unterer Ösophagussphinkter (lower esophageal sphincter)

M.

Musculus

$\mathrm{ml}$

Milliliter

MRT

Magnetresonanztomographie

NANC

nicht-adrenerg-nicht-cholinerg

NO

Stickstoffmonoxid

PACAP

pituitary adenylate cyclase activating polypeptide

PAVK

peripher-arterielle Verschlusskrankheit

PEG

perkutane endoskopische Gastrostomie

PPI

Protononenpumpeninhibitor

SNAP-25

synaptosomal associated protein of 25 kiloDalton

Tab.

Tabelle

U

Units

u. a.

unter anderem

UES

Oberer Ösophagussphinkter (upper esophageal sphincter)

v. a. vor allem

VIP

vasoactive intestinal Polypeptide

z. B.

zum Beispiel

Z. n.

Zustand nach 


\section{II.2 Abbildungsverzeichnis}

Abb. 1: Schluckakt in den verschiedenen chronologischen Phasen: A - E ............1

Abb. 2: Pharyngoösophagealer Übergang, dorsale Ansicht.................................4

Abb. 3: Übergang von Ösophagus und Magen: Unterer Ösophagus mit Kardia .....7

Abb. 4: Interaktion am LES zwischen Neuron, Cajal-Zelle und Muskelzelle.............9

Abb. 5: Radiologischer Befund einer Bariumbrei-Kontrastuntersuchung...............12

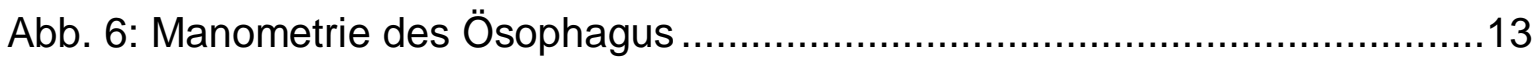

Abb. 7: Physiologische Reizweiterleitung an der cholinergen Synapse ...............19

Abb. 8: Wirkmechanismus von Botulinumtoxin an der cholinergen Synapse ........19

Abb. 9: Zur Applikation von Botulinumtoxin benötigte Materialien.........................25

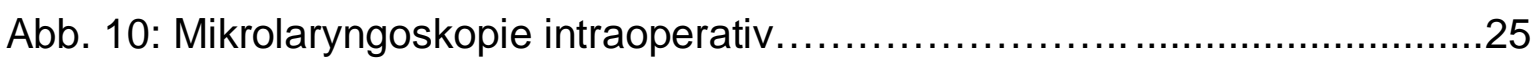

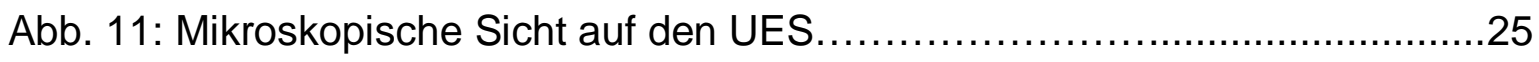

Abb. 12 und 13: Endoskopische Sicht auf den distalen Ösophagus ...................27

Abb. 14: Begleiterkrankungen des Patientenkollektivs ........................................32

Abb. 15: Medikation des Patientenkollektivs …………........................................

Abb. 16: Verteilung der Symptome in der Gruppe UES......................................36

Abb. 17: Verteilung der Symptome in der Gruppe LES ......................................37

Abb. 18: Anzahl der Patientenvorstellungen in Prozent..........................................41

Abb. 19: Videofluoroskopie des UES vor und nach der Behandlung mit 


\section{II.3 Tabellenverzeichnis}

Tab. 1: Differenzialdiagnosen der cricopharyngealen Achalasie.

Tab. 2: Charakteristika des Patientenkollektivs mit unterschiedlicher

Dysphagie 30

Tab. 3: Dauer der Beschwerdesymptomatik .38

Tab. 4: Vorangegangene Therapien bei Dysphagie .40

Tab. 5: Dosierung von Botulinumtoxin A bei Erstinjektion in Prozent. .42

Tab. 6: Gruppe UES, Dauer in Tagen bis zur erneuten Vorstellung zwischen der ersten und zweiten Injektion

Tab. 7: Gruppe UES, Dauer in Tagen bis zur erneuten Vorstellung zwischen der zweiten und dritten Injektion.....

Tab. 8: Gruppe LES, Dauer in Tagen bis zur erneuten Vorstellung zwischen der ersten und zweiten Injektion .45

Tab. 9: Gruppe LES, Dauer in Tagen bis zur erneuten Vorstellung zwischen der zweiten und dritten Injektion .45

Tab. 10: Gruppe LES, Dauer in Tagen bis zur erneuten Vorstellung zwischen der dritten und vierten Injektion

Tab. 11: Dosismodifikation zwischen den Injektionen .46

Tab. 12: Intervallmodifikation zwischen den Injektionen .46

Tab. 13: Dauer des Wirkungseintritts nach Botulinumtoxin-A-Injektion .48

Tab. 14: Übereinstimmung von objektivem und subjektivem Therapieergebnis....51

Tab. 15: Übersicht über das Patientenkollektiv der Responder. .53

Tab. 16: Übersicht über das Patientenkollektiv der Non-Responder .54 
1 EINLEITUNG

\section{$1.1 \quad$ Dysphagie}

\subsubsection{Das Symptom Dysphagie und der physiologische Schluckakt}

Das Symptom „Dysphagie“ beschreibt das „Gefühl einer Schluckstörung ohne Schmerzen infolge Passagestörung geschluckter Speisen“" (Herold 2004, S. 357). Einige Ursachen dieser Schluckstörungen lassen sich mit Hilfe der Betrachtung des physiologischen Schluckablaufs erklären, der in der folgenden Abbildung (Abb.) 1 dargestellt ist.

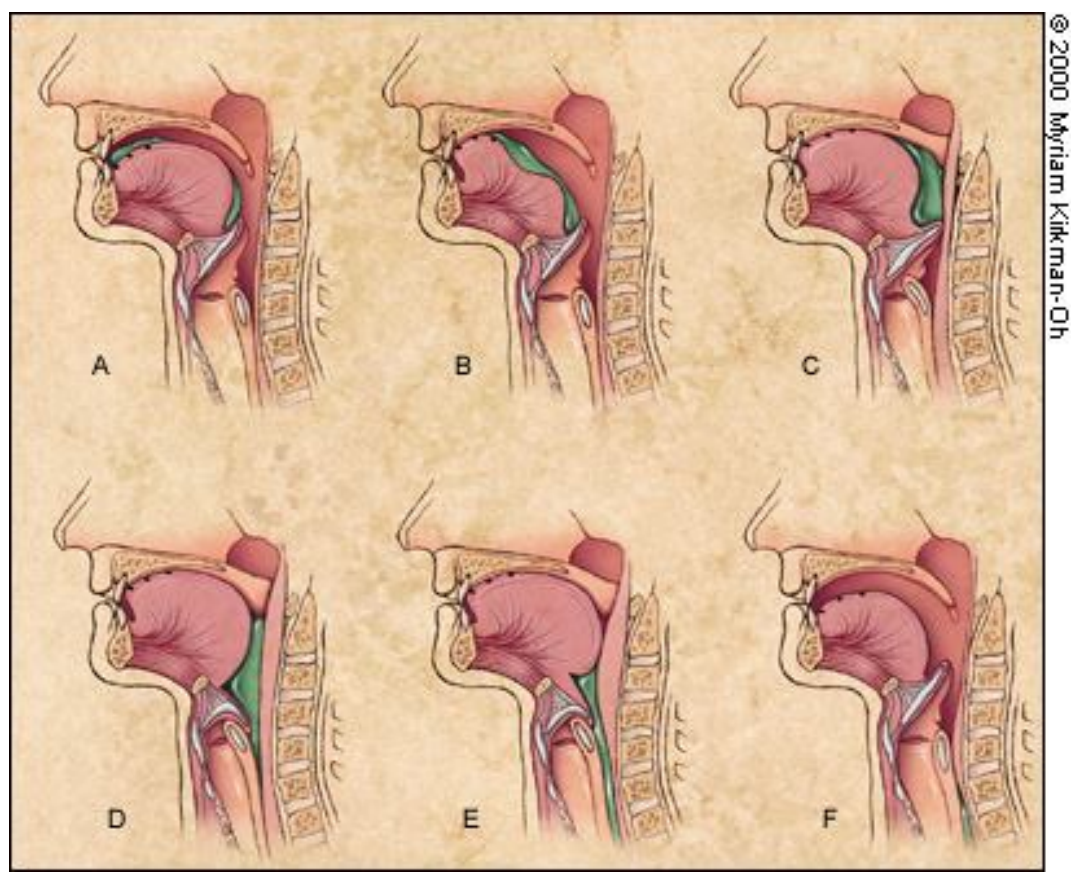

Abb. 1: Schluckakt in den verschiedenen chronologischen Phasen: A - E

(Palmer et al. 2000, S. 2456)

Bild A: Zerkleinern und Einspeicheln der Speise, Bild B: Formung eines Speisebolus, Bild C: Transport des Speisebolus in den Oropharynx und Verschluss des Nasopharynx durch den weichen Gaumen, Bild D: Verschluss des Respirationstrakts durch die Epiglottis und Öffnung des UES, Bild E und F: ösophageale Phase mit Transport des Speisebolus in Richtung Magen

Der Schluckakt lässt sich in einen willkürlichen und einen reflexgesteuerten und damit unwillkürlichen Anteil unterteilen. Es lassen sich zwei Phasen identifizieren, die dem willkürlichen Anteil zugeordnet sind. Die erste, sogenannte „orale Vorbe- 
reitungsphase", bezieht sich auf die sensorische Wahrnehmung der Speise, die Aufnahme über die Lippen in den Mund, das Zerkleinern und Einspeicheln der Speise, sowie das Platzieren des Speisebolus durch Formung des Zungenkörpers. In der zweiten Phase, der "oralen Phase“, wird der Bolus durch Zungenformung in den Oropharnyx transportiert. Anschließend wird in der „pharyngealen Phase" der Schluckreflex ausgelöst. So werden die Nasenhöhle und der Respirationstrakt reflektorisch verschlossen und der Speisebolus wird über den erschlafften oberen Ösophagussphinkter (,upper esophageal sphincter" (UES)) in den Ösophagus transportiert. Im Anschluss an die „pharyngeale Phase“ folgt eine verlängerte Exspiration, wodurch die im Pharynx verbliebenen Speisereste oralwärts transportiert werden. In der „ösophagealen Phase“ befördert die aktive Peristaltik des Ösophagus, welche durch die Wanddehnung beim Schlucken eines Bissens ausgelöst wird, den Bolus durch den Ösophagus und über den relaxierten unteren Ösophagussphinkter („lower esophageal spincter“ (LES)) in den Magen. Die beiden zuletzt genannten Phasen, „pharyngeale“ und „ösophageale Phase“, bilden damit den unwillkürlichen, reflexgesteuerten Anteil des Schluckaktes (Böhme 2003; Müller und Lorenz 2005).

\subsubsection{Unterteilung verschiedener Dysphagieformen aufgrund ihrer anatomischen Zugehörigkeit}

Der Schluckakt ist hoch komplex aufgebaut. Einerseits anatomisch-morphologisch, durch die Kreuzung von Luft- und Speisewegen, und andererseits physiologisch-funktionell durch die Verflechtung von Willkür- und Reflexmotorik (Walther 1998). Dieser Vorgang wird über sechs Hirnnerven und 26 Muskeln gesteuert (Donner et al. 1985).

Der funktionelle Ablauf dieser unterschiedlichen Nerven und Muskeln bedarf einer präzisen Koordination, da beispielsweise die durch den Schluckreflex ausgelöste pharyngeale Phase des Schluckaktes innerhalb von maximal einer Sekunde abläuft (Böhme 2003). Diese Koordination übernimmt das Schluckzentrum, das im kaudalen Hirnstamm zu finden ist. Hier laufen sowohl die afferenten Nerven aus Zungengrund, dorsaler Epiglottis und vorderem Gaumenbogen zusammen, als auch die efferenten Nerven, welche ihre Zielgebiete von hier aus ansteuern 
(Donner et al. 1985). Aufgrund der physiologischen Komplexität ergeben sich die unterschiedlichsten Ursachen einer Dysphagie, die anhand ihrer anatomischen Lage nach Lokalisation unterteilt werden.

Im Allgemeinen werden Schluckstörungen in oropharyngeale und ösophageale Dysphagien unterteilt. Oropharyngeale Dysphagien bereiten Schluckbeschwerden zu Beginn des Schluckaktes oder zeigen sich als Penetration und/oder Aspiration während der Nahrungsaufnahme mit Hustenanfällen. Hingegen bezeichnet die ösophageale Dysphagie eine Passagebehinderung für feste und flüssige Speisen, die sich häufig in Form von Würgereiz und Erbrechen äußert (Böhme 2003; Pschyrembel 2007).

\subsubsection{Oberer Ösophagussphinkter (UES)}

\subsubsection{Anatomie des Ösophagus und des UES}

Der Ösophagus ist ein muskulöser Schlauch, der abhängig vom Kontraktionszustand der Muskelwand unterschiedlich weit und beim Erwachsenen im Durchschnitt 25 bis 30 Zentimeter $(\mathrm{cm})$ lang ist. Er ist, wie der gesamte Rumpfdarm, von innen nach außen aus vier Schichten aufgebaut: die Tunica mucosa, die Tela submucosa, die Tunica muscularis und die Tunica adventitia. Die Tunica muscularis ist aus dem Stratum circulare, der inneren Ringmuskulatur, und dem Stratum longitudinale, der äußeren Längsmuskulatur, aufgebaut. Zwischen diesen beiden Muskelschichten liegt der Plexus myentericus, ein Nervengeflecht, welches die Muskelschichten innerviert. Ein weiteres Nervengeflecht stellt der Plexus submucosus dar, der sich zwischen der Tela submucosa und dem Stratum circulare der Tunica muscularis befindet. Der Ösophagus wird über das vegetative Nervensystem innerviert. Zum einen versorgt der Nervus vagus und zum anderen der Sympathicus, aus dem Ganglion cervicothoracicum und dem Plexus aorticus thoracicus, die Nervenplexus des Ösophagus (Frick et al. 1992; Young et al. 2000).

Im oberen Drittel besteht der Ösophagus aus quergestreifter Muskulatur. Diese geht im Verlauf in glatte Muskulatur über, so dass die unteren beiden Drittel aus 
glatter Muskulatur aufgebaut sind. Die glatte Muskulatur wird über die beiden oben beschriebenen Nervenplexus innerviert. Die quergestreifte Muskulatur wird hingegen über die Rami oesophageales der Nervi laryngeus superior et recurrens direkt versorgt (Frick et al. 1992; Young et al. 2000).

Der UES, direkt am Beginn des Ösophagus, bildet die engste Stelle des Ösophagus mit einer Dehnung bis etwa 1,4 cm. Er setzt sich aus mehreren Muskeln zusammen, die der unteren Pharynxmuskulatur und der Muskulatur des oberen Ösophagus angehören. Die muskuläre Hauptkomponenete bildet der Musculus (M.) cricopharyngeus. Abgesehen vom Schlucken und Erbrechen ist dieser tonisch kontrahiert (siehe Abb. 2) (Frick et al. 1992; Young et al. 2000).

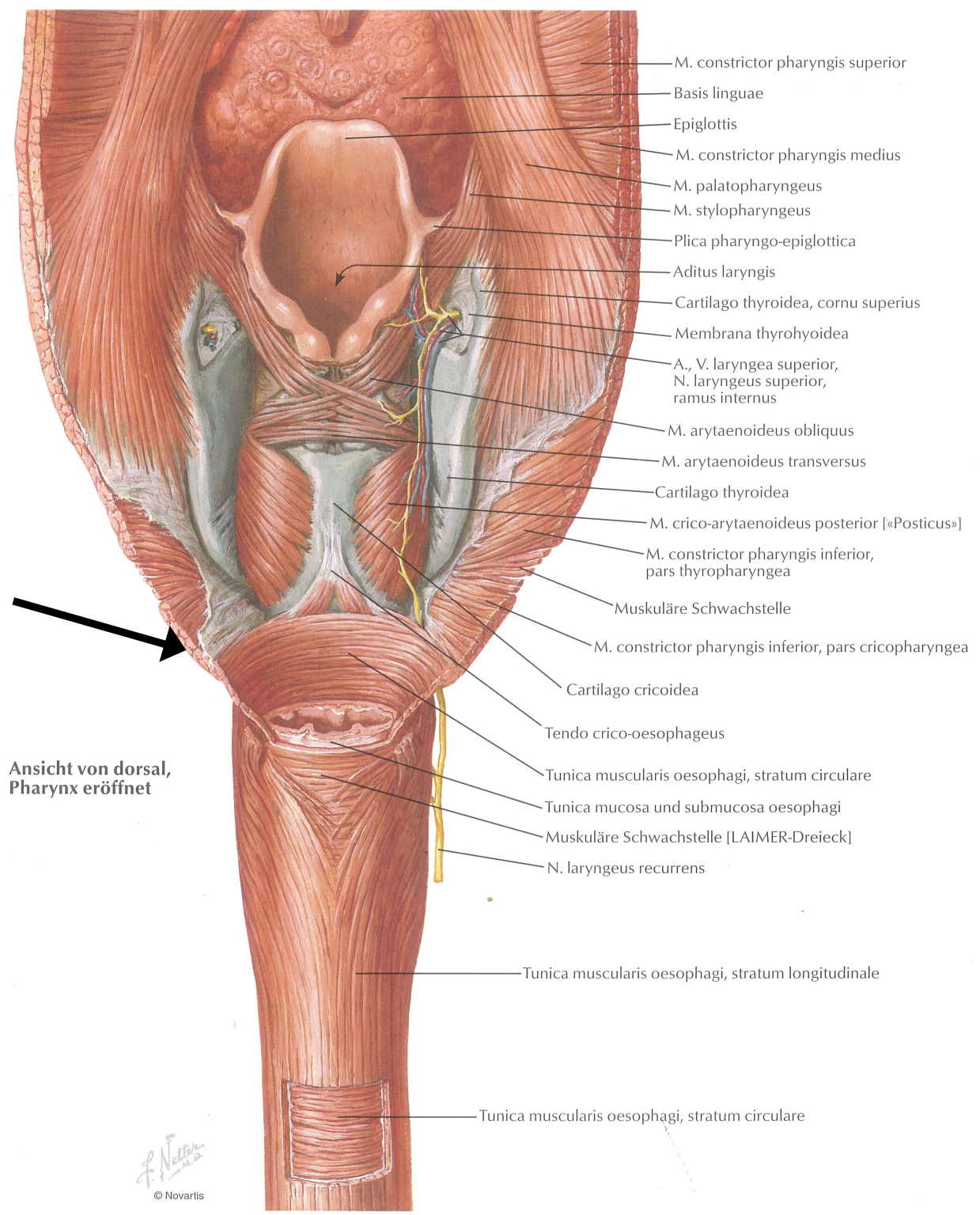

Abb. 2: Pharyngoösophagealer Übergang, dorsale Ansicht, $\rightarrow:$ M. cricopharyngeus (Netter 2000, S. 223) 


\subsubsection{Pathophysiologie und Klinik der „cricopharyngealen Achalasie“}

Ein beobachtetes Problem im Schluckablauf ist die Dysfunktion des UES. Dieses Krankheitsbild wird meist auf eine Hypertrophie des M. cricopharyngeus zurückgeführt und auf Grund dessen als cricopharyngeale Achalasie bezeichnet (Walther 1998). Das Wort „Achalasie“ stammt aus dem Griechischen und wird ins Deutsche als "Unfähigkeit zu erschlaffen" übersetzt (Pschyrembel 2007). Hierfür gibt es zahlreiche mögliche Ursachen wie Tabelle (Tab.) 1 zeigt.

Tab. 1: Differenzialdiagnosen der cricopharyngealen Achalasie.

(nach Walther 1998, S. 721)

\begin{tabular}{|l|l|}
\hline $\begin{array}{l}\text { Differenzialdiagnose der } \\
\text { cricopharyngealen Achalasie }\end{array}$ & \\
\hline Neurogen & Ischämie/Insult \\
\hline & Poliomyelitis \\
& Amyotrophe Lateralsklerose \\
& Multiple Sklerose \\
& M. Parkinson \\
\hline Hirnstammtumore \\
\hline periphere Neuropathien \\
\hline myogen & Dermatomyositis \\
\hline Verschiedenes & Myasthenia gravis \\
\hline & thyreotoxische Myopathie \\
\hline gastroösophageale Refluxkrankheit \\
\hline postoperativ \\
\hline - pharyngolaryngeale Tumore \\
\hline - ventrale HWS \\
\hline - Schädelbasis \\
\hline kongenital \\
\hline externe pharyngoösophageale Kompression \\
\hline
\end{tabular}

Zur Pathophysiologie gibt es drei mögliche Mechanismen:

1. fehlende oder inkomplette Relaxation des UES (Ekberg 1987),

2. eine nicht mit der pharyngealen Kontraktionsphase abgestimmte Relaxationsphase des UES (Dantas et al. 1990),

3. vorzeitiger Verschluss des UES (Quirk und Friedman 1999). 
Wenn sich der UES nicht vollständig oder nicht zeitgerecht öffnet, kommt es zum Aufstau der Speise im Pharynx. Die Folge dieser Penetration ist schließlich starker Hustenreiz bis hin zur Aspiration von Speisen. Eine Aspiration von Speisen kann wiederum zu Pneumonien führen. So klagen die Patienten über ein „Verschlucken“ von Nahrung gefolgt von starkem Hustenreiz, rezidivierenden Pneumonien, wiederholten Fieberepisoden und/oder unfreiwilligem Gewichtsverlust, bedingt durch Infektionen, Verweigern von Nahrung oder problematische Ingestion der Speise (Pototschnig und Thumfart 2004; Walther 1998). Ein weiteres Symptom ist das sogenannte „drooling“, welches auch unter „Speicheln“ bekannt ist. Eine relative Hypersalivation, aufgrund der Beeinträchtigung den eigenen Speichel zu schlucken, ist beispielsweise auch nach Tumorchirurgie im Kopf-Hals-Bereich aufgefallen (Ellies et al. 2002; Laskawi und Ellies 2007).

\subsubsection{Diagnostik der pharyngealen Dysphagie}

Eine ausführliche Anamnese sollte die Art der Dysphagie möglichst genau erfassen können. So sind beispielsweise Fragen nach einer Dysphagie bei festen oder flüssigen Speisen, Bedingungen zur Verstärkung oder Verschlechterung der Dysphagie und Auftreten eines Hustenreizes während des Schluckens wichtig. Neben der Anamnese und einer gründlichen klinischen Untersuchung inklusive Lupenlaryngoskopie und Pharyngoskopie bieten sich eine Vielzahl von apparativen diagnostischen Verfahren an. Als erstes sollte eine dynamische Röntgendarstellung des gesamten Schluckweges von der Mundhöhle bis in den Magen durchgeführt werden. Anschließend kommen pharyngeale Computermanometrie, Videofluoroskopie beziehungsweise (bzw.) Hochfrequenz-Kinematographie, Elektromyographie, Endoskopie und Szintigraphie in abnehmender Gewichtung zum Einsatz. Zur Diagnostik der Aspiration sind Videofluoroskopie bzw. Hochfrequenz-Kinematographie, Szintigraphie und $\mathrm{pH}-$ Metrie die am meisten verbreiteten Verfahren (Walther 1998). In der klinischen Praxis stellen jedoch Endoskopie und Videofluoroskopie die wichtigsten diagnostischen Instrumente dar (Müller und Lorenz 2005). 


\subsubsection{Unterer Ösophagussphinkter (LES)}

\subsubsection{Anatomie und Physiologie des LES}

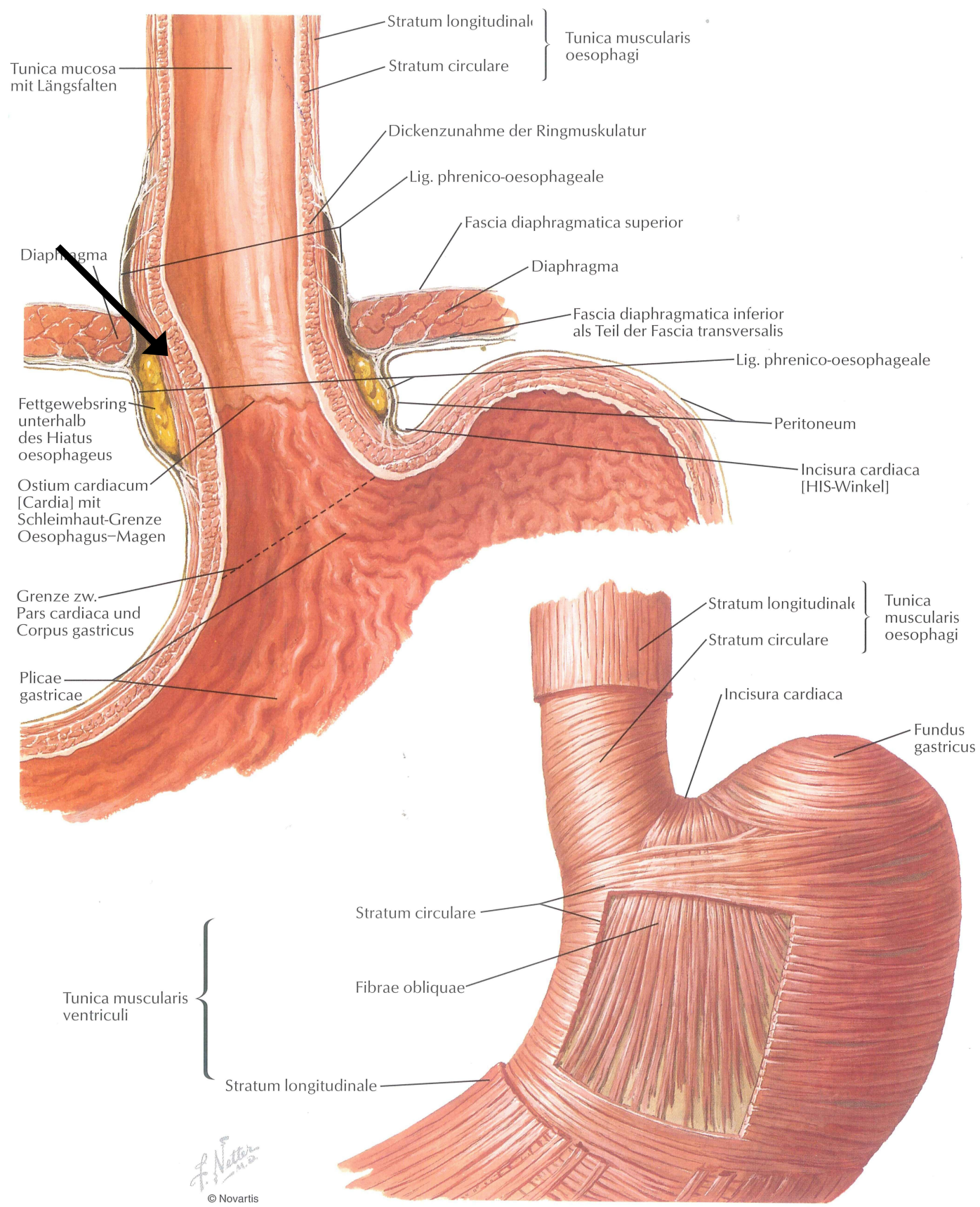

Abb. 3: Übergang von Ösophagus und Magen: Unterer Ösophagus mit Kardia im Längsschnitt und Aufbau der einzelnen Muskelschichten, $\rightarrow$ : LES-Region

(Netter 2000, S. 224)

Der LES liegt im abdominellen Abschnitt des Ösophagus beim Durchtritt durch das Zwerchfell (siehe Abb. 3). Er gehört darüber hinaus zu einer der drei Engen des Ösophagus. Die obere Ösophagusenge bildet der UES, die mittlere entsteht durch 
die unmittelbare Nachbarschaft von linkem Hauptbronchus und Aortenbogen und die untere bildet der LES beim Durchtritt des Ösophagus durch das Zwerchfell. Er ist als "funktioneller Kardiasphinkter" beschrieben. Dieser entsteht durch die Anordnung der Muskelfasern zu einer Art Ringmuskel und durch submuköse und muköse Venen. Darüber hinaus führt der abdominelle Druck durch den sogenannten His-Winkel („Incisura cardiaca“), dem Winkel zwischen Ösophagus und Kardia, zu einem zusätzlichen Verschluss der Kardia (Frick et al. 1992). Der LES ist ebenso wie der UES fortwährend tonisch kontrahiert. Diese Kontraktion wird vor allem (v. a.) durch Acetylcholin (Ach) aus den Ganglienzellen des Plexus myentericus ausgelöst.

Weitere Substanzen, die eine Kontraktion des LES bewirken, sind: $\alpha$-adrenerge Agonisten, Gastrin, Motilin, Somatostatin, Substanz P, proteinreiche Kost, sowie parakrine Mediatoren (Histamin, Prostagladin $F_{2 \alpha}$ ) (Young et al. 2000).

Während des Schluckens öffnet sich der LES reflektorisch bereits zu Beginn des Schluckaktes. Dies geschieht über eine rezeptive Relaxation durch NANC-Fasern (nicht-adrenerge-nicht-cholinerge Fasern) aus dem Plexus myentericus. Teilweise wird auch über die Cajal-Zelle (,interstitial cell of Cajal“) eine Relaxation vermittelt. Cajal-Zellen innerhalb des Gastrointestinaltraktes haben unterschiedliche Funktionen, unter anderem (u. a.) Schrittmacherfunktion und neuromuskuläre Signaltransduktion (Kraichely und Farrugia 2006). Die Transmitter der NANC-Fasern sind Stickstoffmonoxid (NO), Vasoactive intestinal polypeptid (VIP) und Adenosintriphosphat (Goyal und Chaudhury 2008; Young et al. 2000).

Weitere Substanzen die eine Relaxation des LES bewirken sind: B-adrenerge Agonisten, Sekretin, Cholecystokinin, Progesteron, glucose-dependent insulinreleasing peptide bzw. gastric inhibitory polypeptide (GIP), pituitary adenylate cyclase activating polypeptide (PACAP), fettreiche Kost, sowie parakrine Mediatoren (Prostaglandin $\mathrm{I}_{2}$, Prostaglandin $\mathrm{E}_{2}$, Dopamin) (siehe Abb. 4) (Frick et al. 1992; Kraichely und Farrugia 2006; Young et al. 2000). 


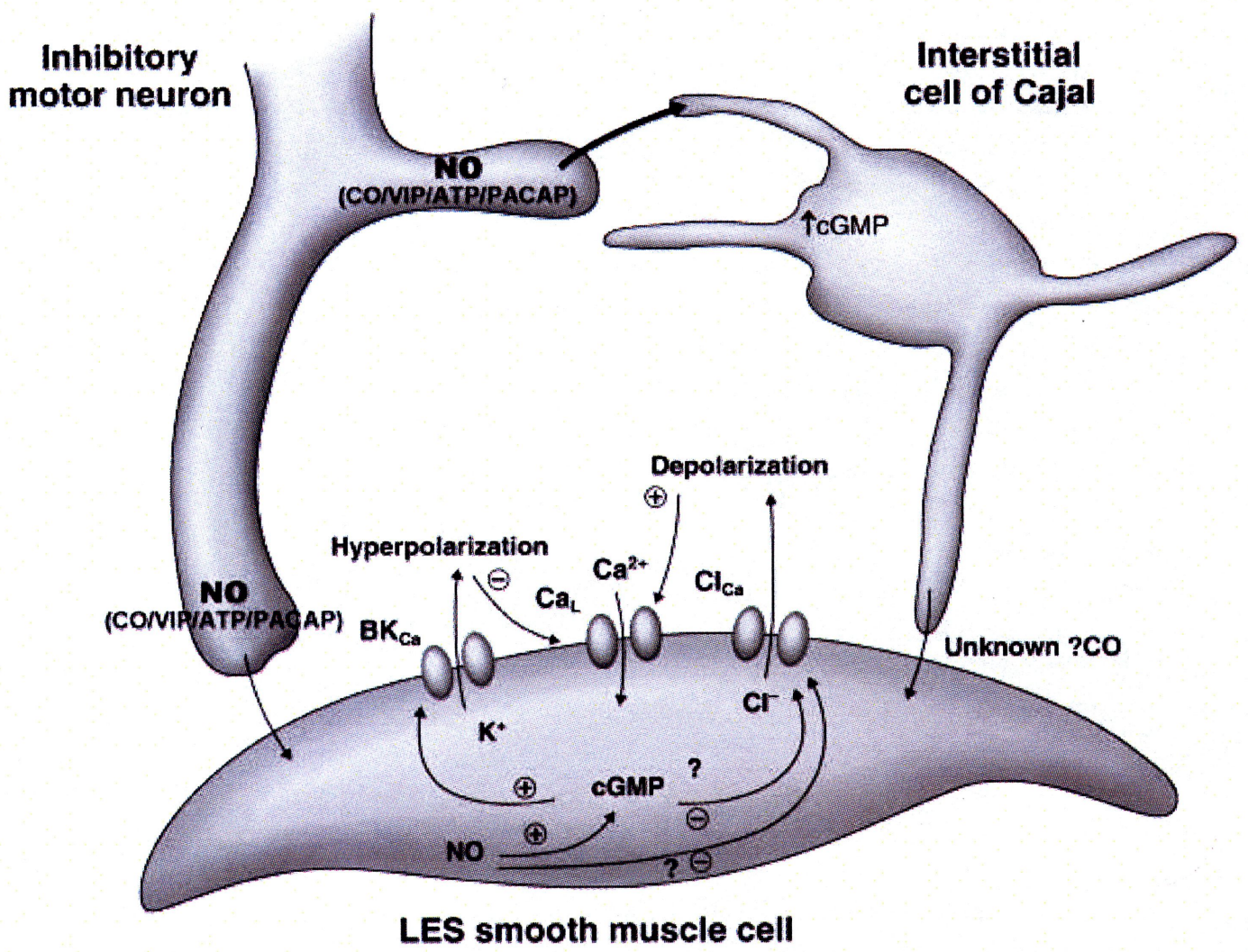

Abb. 4: Interaktion am LES zwischen Neuron, Cajal-Zelle und Muskelzelle:

Mediatoren der Relaxation, v. a. NO, gelangen in die Cajal-Zelle und Muskelzelle.

Dort führen sie über eine Hyperpolarisation der Muskelzelle zur Relaxation

(Kraichely und Farrugia 2006, S. 217)

\subsubsection{Pathophysiologie und Klinik der Achalasie}

Im Bereich des LES ist die Achalasie eine bedeutende Erkrankung. Bei diesem Krankheitsbild zeigt sich eine unzureichende schluckreflektorische Erschlaffung des unteren Ösophagussphinkters. Häufig ist darüber hinaus die propulsive $\mathrm{Pe}$ ristaltik des tubulären Ösophagus gestört (Quirk und Friedman 1999). Die Achalasie kann in drei Stadien nach der Klassifikation nach Allgöwer und Mitarbeiter eingeteilt werden (Hornbostel 1986). Im "Stadium I" ist keine Dilatation des proximalen Ösophagus sichtbar, es besteht eine tertiäre spärliche Motilität und der Tonus im Bereich des LES ist normal bis erhöht mit unkoordinierter Erschlaffung. Im „Stadium II“ zeigt sich eine deutliche Dilatation des proximalen Ösophagus, die Motilität ist kaum mehr vorhanden und der Tonus im LES-Bereich ist normal bis erhöht mit meist unmöglicher Öffnung. Die Dilatation im „Stadium III“ ist ohne Mo- 
tilität und unmöglicher Öffnung des LES bei normal bis erhöhtem Ruhetonus (Hornbostel 1986).

In chirurgischen Resektaten wurde eine Degeneration und Entzündungsreaktion, v. a. mit zytotoxischen T-Lymphozyten, der Ganglienzellen im Plexus myentericus, gefunden (Kraichely und Farrugia 2006; Quirk und Friedman 1999). Daneben ist ein selektiver Verlust postganglionärer inhibitorischer Neurone, die NO und VIP enthalten, sowie ein Verlust der NO-Synthetaseaktivität aufgefallen (Schwörer 2004). Durch den Verlust dieser Neurone wird eine unzureichende Relaxation des LES hervorgerufen. Da postganglionäre stimulatorische Neurone nicht von dem Zelluntergang betroffen sind, überwiegt die cholinerge Stimulation und es kommt zu einem erhöhten Ruhetonus im LES (Kraichely und Farrugia 2006).

Aufgrund dieser Pathophysiologie lässt sich die Symptomatik gut ableiten. Klinisch beschreiben die Patienten eine Dysphagie für feste und flüssige Nahrung, die sie mit verschiedenen Manövern zu lindern versuchen. Zu diesen Manövern zählen Essen im Stehen, Strecken des Halses, ein nach Hintenziehen der Schultern und Trinken von stark kohlensäurehaltigen Getränken. Durch eine Retention von Speisen im Ösophagus kann es zu Halithosis (Mundgeruch) kommen. Bis zu 90\% der Patienten geben Regurgitationen an, das heißt ein Zurückfließen von unverdauter Nahrung. Diese können während oder kurz nach dem Essen auftreten. Im fortgeschrittenen Stadium treten Regurgitationen ebenfalls während der Nacht auf, so dass Speichel und Nahrungsreste am nächsten Morgen auf dem Kopfkissen entdeckt werden. Komplikationen im Sinne einer Aspiration können arüber hinaus durch Regurgitationen auftreten. So klagen diese Patienten über rezidivierende bronchopulmonale Symptome. In bis zu 50\% der Fälle treten thorakale Schmerzen auf, die sich in retrosternalem Druckgefühl, teilweise mit Ausstrahlung in den Hals, den Kiefer oder in die Arme äußern. Sodbrennen ist ein weiteres Symptom, welches Patienten angeben. Schließlich zeigt sich bei einem Fortschreiten der Erkrankung ein zunehmender Gewichtsverlust (Renz-Polster und Braun 2001; Schwörer 2004).

Die Ursache für diese Zelluntergänge ist letztlich noch immer unklar. Die oben beschriebene primäre Form der Achalasie, die mit circa $90 \%$ die häufigste Variante 
darstellt, wird von der sekundären Achalasie, welche andere Krankheitsbilder betrifft, die mit einer Achalasiesymptomatik assoziiert sind, unterschieden (Gockel et al. 2005). Bei der primären Achalasie werden genetische Determinationen, neurotoxische Agenzien bzw. Infektionen mit Herpesviren, hierbei v. a. mit dem Varizella-zoster-Virus und Herpes simplex Typ 1, sowie Masernviren und Ischämiefolgen als Ursache diskutiert. Ebenfalls ist der Nachweis von Autoantikörpern gegen den myenterischen Nervenplexus bei Achalasiepatienten gelungen (Di Nardo et al. 2008; Park und Vaezi 2005).

Zu der sekundären Achalasie werden u. a. das sogenannte „Allgrove-Syndrom“, das aus einer Trias aus „Achalasie, Alakrimie und Nebennierenrindeninsuffizienz“ besteht, gezählt (Kasar et al. 2007). Eine weitere Erkrankung, die zur Achalasie führen kann ist die Chagas-Krankheit, die auf den Erreger Trypanosoma cruzii zurückgeht. Weitere Ursachen sind zum Beispiel (z. B.) die Langerhans-Histiozytose und die Amyloidose (Pohl und Tutuian 2007). Eine sogenannte „Pseudoachalasie“ entsteht durch Tumore oder deren Metastasen im Bereich der Kardia, wie beispielsweise Adenokarzinome, Bronchialkarzinome, Lymphome, oder durch andere Obstruktionen wie Fibrosen bzw. chirurgische Interventionen, wie Antireflux-Operationen und "gastric banding“. Zudem kann die Achalasie auch paraneoplastische Ursachen, beispielsweise aufgrund eines Bronchialkarzinoms, haben (Gockel et al. 2005; Herold 2004).

\subsubsection{Diagnostik der Achalasie}

Die ausführliche Anamnese und klinische Untersuchung haben auch bei der ösophagealen Achalasie einen hohen Stellenwert. Daneben sind apparative Verfahren zur weiteren Diagnostik unerlässlich. So zeigt sich in der Bariumbrei-Kontrastuntersuchung des Ösophagus eine sogenannte „Sektglasform“, die infolge des prästenotisch weitgestellten Ösophaguses und der engen Stenose, die nur wenig Kontrastmittel durchlässt, zu Stande kommt (siehe Abb. 5) (Herold 2004). 


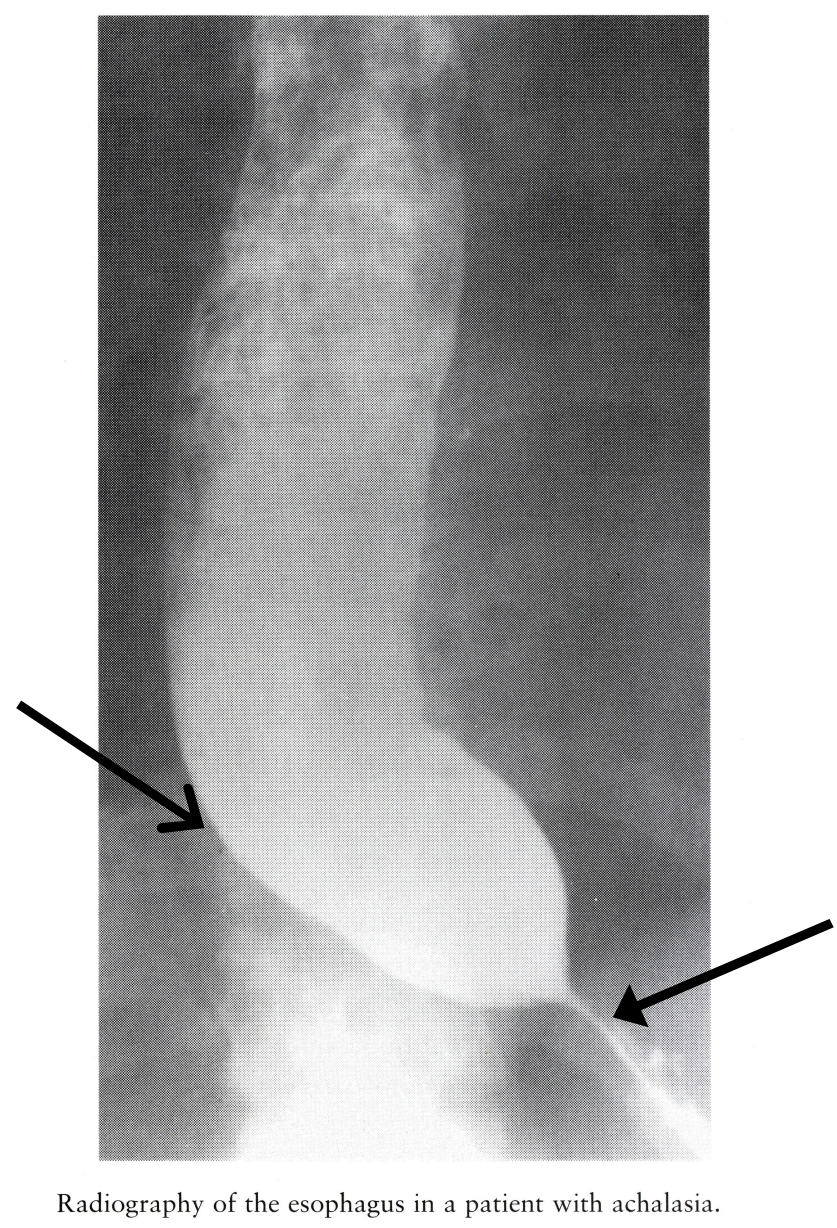

Abb. 5: Radiologischer Befund einer Bariumbrei-Kontrastuntersuchung bei fortgeschrittener Achalasie entsprechend „Stadium II“ bis „III“, Bild der „Sektglasform“ $\rightarrow$ : prästenotische Dilatation des Ösophagus und $\rightarrow$ : fadenförmige Stenose im Bereich des LES (Stendal 1997, S. 36)

Weiterführend kommen Ösophagogastroduodenoskopie und Manometrie zum Einsatz. Durch die Ösophagogastroduodenoskopie wird die Stenose beurteilt, sowie Biopsien zum Karzinomausschluss entnommen (Herold 2004).

Durch Applikation von Glyceroltrinitrat in die LES-Region kann ebenfalls ein diagnostischer Anhalt auf Achalasie gewonnen werden, da bei primärer Achalasie eine Relaxation erfolgt (Schwörer 2004). 


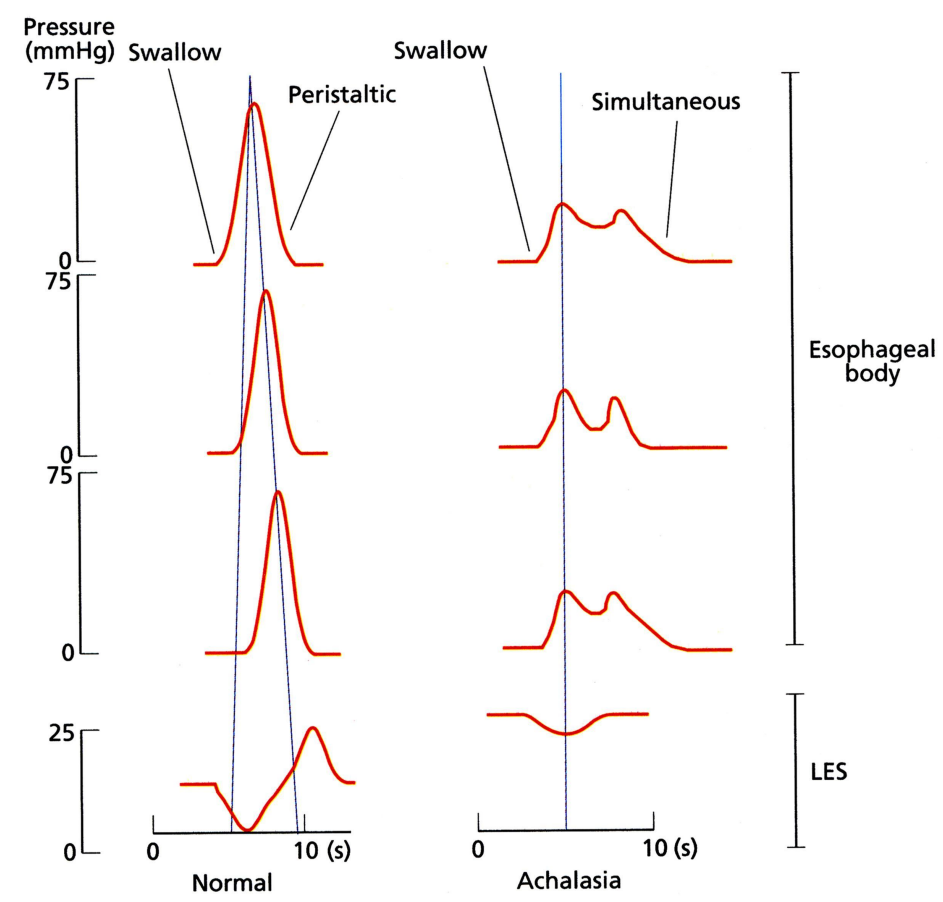

Schematic representation of manometric profile in a patient with achalasia. Reproduced from Smout, 1992 with permission from Wrightson Biomedical Publishing Ltd.

Abb. 6: Manometrie des Ösophagus:

Links physiologische Abb. und rechts Abb, im Rahmen einer Achalasie während eines Schluckversuchs. Die dargestellten Wellen stellen Druckunterschiede, die auf Grund einer peristaltischen Kontraktion zustande kommen, innerhalb der unterschiedlichen Höhen im Ösophagus von oben nach unten dar. Die unterste Welle stellt den LES dar. Im Vergleich zur physiologischen Abb. stellt sich eine geringere und unregelmäßigere Druckwelle dar, da die Ösophagusmuskulatur unkoordiniert und schwach arbeitet. Der LES zeigt darüber hinaus einen wesentlich höheren Ruhedruck mit nur unzureichender Relaxation.

(Stendal 1997, S. 37)

Eine fehlende oder mangelnde Relaxation des LES kann mit Hilfe der Manometrie ermittelt werden. Es wird ein normaler bis erhöhter Ruhedruck über $8 \mathrm{mmHg}$ im Bereich des LES erwartet. In einigen Fällen mit stark erhöhtem Ruhedruck im LES steigt auch der basale intraösophageale Druck an. Teilweise zeigt sich darüber hinaus das Bild einer gestörten Peristaltik bis hin zur Aperistalsis während des Schluckakts (siehe Abb. 6). Die Manometrie gilt daher auch als "Goldstandard“ in der Diagnostik der Achalasie (Pohl und Tutuian 2007). Zum Ausschluss eines tumorösen Geschehens sollte eine Endosonographie, sowie eventuell weitere bildgebende Verfahren des Ösophagus, z. B. eine Computertomographie (CT), durchgeführt werden (Schwörer 2004; Vaezi und Richter 1999). 


\subsection{Konservative und operative Therapieverfahren bei Dysphagie}

\subsubsection{Behandlungsmöglichkeiten des UES (oberer Ösophagussphinkter)}

Zur Therapie der oropharyngealen Dysphagie sind in der Literatur zahlreiche, unterschiedliche Optionen beschrieben. Diese werden allgemein in drei Gruppen unterteilt (Müller und Lorenz 2005; Pototschnig und Thumfart 2004; Singh und Hamdy 2005; Walther 1998):

1. konservative Methoden (professionelle Schlucktherapie mit Mendelsohn-Manöver),

2. endoskopische Methoden (intramuskuläre Botulinumtoxininjektion, Dilatation),

3. operative Methode (cricopharyngeale Myotomie).

In der konservativen Therapie nimmt die professionelle Schlucktherapie eine zentrale Rolle ein. Dabei werden restituierende Verfahren, wie z. B. Larynxelevationsübungen, zu denen u. a. das Mendelsohn-Manöver zählt, kompensatorische, zu denen Halteänderungen und Schlucktechniken zählen, und adaptive Verfahren, wie z. B. das Verändern der Viskosität der Speisen, unterschieden (Müller und Lorenz 2005; Walter 1998).

Bei den endoskopischen Therapien steht die intramuskuläre Injektion mit Botulinumtoxin zur Verfügung. Dieses Verfahren lässt sich transoral mittels starrer Ösophagoskopie oder flexibler Endoskopie oder auch perkutan mit Hilfe der Elektromyographie-, CT- oder Videofluoroskopie-gesteuerten Injektion durchführen (Laskawi 1998; Moerman 2006). Eine weitere Option stellt die pneumatische Dilatation dar. Diese wird nach einem ähnlichen Verfahren wie die pneumatische Dilatation des unteren Ösophagussphinkters durchgeführt. Es sind bisher zwei Möglichkeiten bekannt, eine anterograde und eine retrograde. Bei der anterograden pneumatischen Dilatation ist der Zugangsweg oral. Der retrograde Zugangsweg kann ausschließlich bei liegender perkutaner endoskopischer Gastrostomie- 
Sondeneinlage, der so genannten PEG-Sondeneinlage, durchgeführt werden. Hierbei wird das Endoskop über die PEG-Anlage in den Magen eingeführt und gelangt schließlich entlang des Ösophagus zur Stenose im Bereich des UES. Diese wird mit Hilfe eines dünnen Drahtes sondiert und bis zum Mund vorgeschoben. Über diesen Draht gelangt schließlich ein zweites Endoskop auf dem anterograden Weg zur Stenose. Aufgrund der geringen Erfahrungswerte befindet sich diese Therapieoption noch in der experimentellen Phase (Bueno et al. 2001; Hatlebakk et al. 1998; Lew et al. 2004; Steele et al. 2007).

Zu den chirurgischen Verfahren zählt die Myotomie des UES. Dieses Verfahren wird entweder konventionell über den Zugang einer lateralen Collotomie oder laserchirurgisch mit dem Kohlenstoffdioxid-Laser über den transoralen Weg durchgeführt. Entscheidend für einen Therapieerfolg ist hierbei die vollständige Durchtrennung aller Muskelfasern, weshalb eine Kontrolle mit Hilfe eines Mikroskops empfohlen wird. Zur Aspirationsprophylaxe stehen neben der cricopharyngealen Myotomie auch andere operative Verfahren zur Verfügung, die bei entsprechenden Lähmungen additiv eingesetzt werden können. Ein Beispiel wäre die Epiglotto- bzw. Aryepiglottopexie. Bei dieser Methode wird eine Trennung von Luft- und Speiseweg sichergestellt, was allerdings die Notwendigkeit einer Trachealkanüle erfordert (Pototschnig und Thumfart 2004; Singh und Hamdy 2005; Walther 1998).

\subsubsection{Behandlungsmöglichkeiten des LES (unterer Ösophagussphinkter)}

Bei der Behandlung der Achalasie stehen verschiedene Therapieprinzipien, die eine Verminderung des Druckes des LES bewirken, zur Auswahl. Im Allgemeinen kann man diese Optionen in drei Gruppen einteilen (Herbella et al. 2008; Lake und Wong 2006; Pehlivanov und Pasricha 2006; Pohl und Tutuian 2007; Vaezi und Richter 1999):

1. medikamentöse Methoden (Calcium-Antagonisten, Nitrate, Sildenafil),

2. endoskopische Methoden (intramuskuläre Botulinumtoxininjektion, Dilatation),

3. operative Methode (Myotomie nach Gottstein-Heller). 
In der medikamentösen Therapie bietet sich eine orale Einnahme von CalciumAntagonisten und Nitraten an. Diese Medikamente werden meist etwa 30 bis 60 Minuten vor der Mahlzeit sublingual eingenommen und können in 50 bis $90 \%$ der Fälle zu einer Senkung des Sphinkterdrucks führen. Bei den Calcium-Antagonisten sind v. a. die vom Dihydropyridin-Typ, z. B. Nifedipin, die Vertreter mit der besten klinischen Wirkung. Verapamil und Diltiazem senken zwar ebenfalls den Druck im LES, zeigen allerdings eine geringere klinische Wirkung. Unter den Nitraten wird Isosorbiddinitrat bevorzugt. Nachteilig ist die blutdrucksenkende Wirkung dieser Medikamente, so dass sie bei Patienten mit tendenziell hypotonen Blutdruckwerten nur bedingt zum Einsatz kommen können. In der Praxis wurde oft die Entwicklung einer Toleranz gegenüber diesen Medikamenten beobachtet, so dass sich damit keine Langzeiterfolge erzielen lassen (Grübel et al. 2008; Lake und Wong 2006; Vaezi und Richter 1999). Bei Sildenafil, einem Phosphodiesterase-5-Hemmstoff, wurde darüber hinaus eine alternative pharmakologische Wirkung beobachtet, ähnlich zu denen von Nifedipin und Isosorbiddinitrat (Bortolotti et al. 2000).

Ferner werden in der Therapie der Achalasie die endoskopischen Methoden erfolgreich durchgeführt. Dabei steht die Behandlung der intramuskulären Botulinumtoxininjektion im LES-Bereich zur Verfügung, Goldstandard ist jedoch die pneumatische Ballondilatation (Schwörer 2004). Bei diesem Verfahren wird über den endoskopischen Weg transoral unter radiologischer Kontrolle eine Ballonsonde auf Höhe des LES eingeführt und mit einem pneumatischen Druck für wenige Minuten aufgedehnt (Da Silveira und Rogers 2002).

Als operative Therapieoption steht die Myotomie nach Gottstein-Heller, die offen oder laparoskopisch, oft über den thorakoskopischen Weg durchgeführt werden kann, zur Verfügung. Indikationen für diese Operation sind: Versagen mehrerer Dilatationsbehandlungen, Rezidive, mangelnde Kooperation bzw. Wunsch des Patienten und junge Patienten, wie z. B. Kinder (Müller 2004). Bei dieser Operation wird die komplette Durchtrennung des LES unter Erhaltung der Mukosa durchgeführt. Aufgrund einer sehr hohen Rate von bis zu 36\% Refluxerkrankten postoperativ kann dieses Verfahren mit einer Fundoplikatio kombiniert werden. Eine Fundoplikatio stellt eine operative Methode zur Reduzierung des gastroöso- 
phagealen Reflux dar. Bei diesem kombinierten Verfahren zeigt sich bei über $80 \%$ der Patienten eine Besserung der Dysphagie auch fünf Jahre postoperativ, ähnlich der Erfolgsrate bei der alleinigen Myotomie, und ein Auftreten eines gastroösophagealen Refluxes bei bis zu 22,4\% auch 15 Jahre nach einer Operation (Csendes et al. 2006; Herbella et al. 2008; Rebecchi et al. 2008; Vaezi und Richter 1999).

\subsection{Botulinumtoxin}

\subsubsection{Therapie mit Botulinumtoxin}

Die ersten Publikationen zum Krankheitsbild des Botulismus wurden im Jahr 1817 in den „Tübingern Blättern für Medizin und Arzneykunde“ von Kerner und Steinbuch veröffentlicht. Diese systematisch-klinische Beschreibung bezog sich auf eine Wurstvergiftung. Allerdings wurde die Beobachtung, dass ein toxinproduzierender Anaerobier für die Lebensmittelvergiftung verantwortlich sein könnte, erst 1897 durch van Ermengem gemacht (Nüßgens 2004). Mittlerweile ist die Ursache des Botulismus besser erforscht und man setzt das dafür verantwortliche Botulinumtoxin darüber hinaus therapeutisch ein.

Der Pionier der Botulinumtoxintherapie, der amerikanische Ophthalmologe Dr. A. B. Scott vom "Smith Kettlewell Eye Research Institute", injizierte Ende der siebziger Jahre des vorherigen Jahrhunderts erstmals Botulinumtoxin A, eines der sieben bekannten Botulinumtoxine, bei einem Menschen; damals, um eine konservative Therapie zum Strabismus zu finden (Scott 1980). Seitdem wurde dieser Wirkstoff immer umfassender untersucht und für weitere Indikationen zugelassen. Aktuell ist Botulinumtoxin A unter vier verschiedenen Produkten, Botox ${ }^{\circledR}$ (Allergan, Irvine, CA, USA), Xeomin ${ }^{\circledR}$ (Merz, Frankfurt/Main, Deutschland), Dysport ${ }^{\boxplus}$ (Ipsen Pharma, Paris, Frankreich) und Vistabe ${ }^{\circledast}$ (Allergan, Irvine, CA, USA) in Deutschland erhältlich.

Botox ${ }^{\circledR}$ beispielsweise ist für ein weites Spektrum an Krankheitsbildern zugelassen. U. a. zählen dazu: Blepharospasmus, hemifazialer Spasmus und koexistie- 
rende lokale Dystonien, Torticollis spasmodicus, dynamische Spitzfußstellung infolge Spastizität bei infantiler Zerebralparese, fokale Spastizität der Hand bei erwachsenen Schlaganfallpatienten und primäre Hyperhidrosis axillaris (Rote Liste 2009). Allerdings werden die Botulinumtoxinpräparate in weiteren Gebieten als "off-label-use" genutzt, das heißt es wird eine Therapie mit diesem zugelassenen Medikament bei einer nicht zugelassenen, jedoch wissenschaftlich begründeten Indikation durchgeführt. Botulinumtoxin A wird u. a. als „off-label-use“ im Ösophagus erfolgreich therapeutisch genutzt, wo es sowohl im Bereich des oberen als auch des unteren Ösophagussphinkters im Rahmen von Dysphagie erfolgreich eingesetzt wird (Kim et al. 2006; Pasricha et al. 1994). Weitere Anwendungen im gastroenterologischen Bereich sind Gastroparese, Dysfunktion des Sphinkter Oddi und Analfissuren (Friedenberg et al. 2004). Im Bereich der Hals-NasenOhrenheilkunde sind Bruxismus, Hypersalivation, Hyperlakrimation, gustatorisches Schwitzen, intrinsische Rhinitis und Aphonie nach Tumorchirurgie im Larynxbereich weitere verbreitete Anwendungsgebiete (Laskawi 2008; Laskawi und Ellies 2007).

\subsubsection{Wirkungsmechanismus der Botulinumtoxine}

Botulinumtoxin ist das potenteste bekannte Gift (Breit und Heckmann 2000). Es wird von einem grampositiven, stäbchenförmigen und obligat anaeroben Bakterium (Clostridium botulinum) gebildet. Zum jetzigen Zeitpunkt sind sieben verschiedene Serogruppen, A bis $G$, bekannt, wobei nur die Typen der Serogruppen $A, B, E$ und $F$ humantoxisch sind (Kreyden et al. 2000).

Die Wirkung von Botulinumtoxin entfaltet sich über eine Blockade der ACh-Ausschüttung an cholinergen Synapsen (siehe Abb. 8). ACh ist ein Transmitter, welcher in der terminalen Nervenendigung cholinerger Neurone synthetisiert und in synaptischen Vesikeln, den so genannten Endosomen, gespeichert wird. Eine neuronale Stimulation der präsynaptischen Nervenzelle cholinerger Neurone führt zur Exozytose des gespeicherten ACh in den synaptischen Spalt, wo es zur postsynaptischen Membran diffundieren kann, um den muskarinergen oder nikotinergen Rezeptor zu aktivieren (siehe Abb. 7) (Greger 2000; Kreyden et al. 2000). 


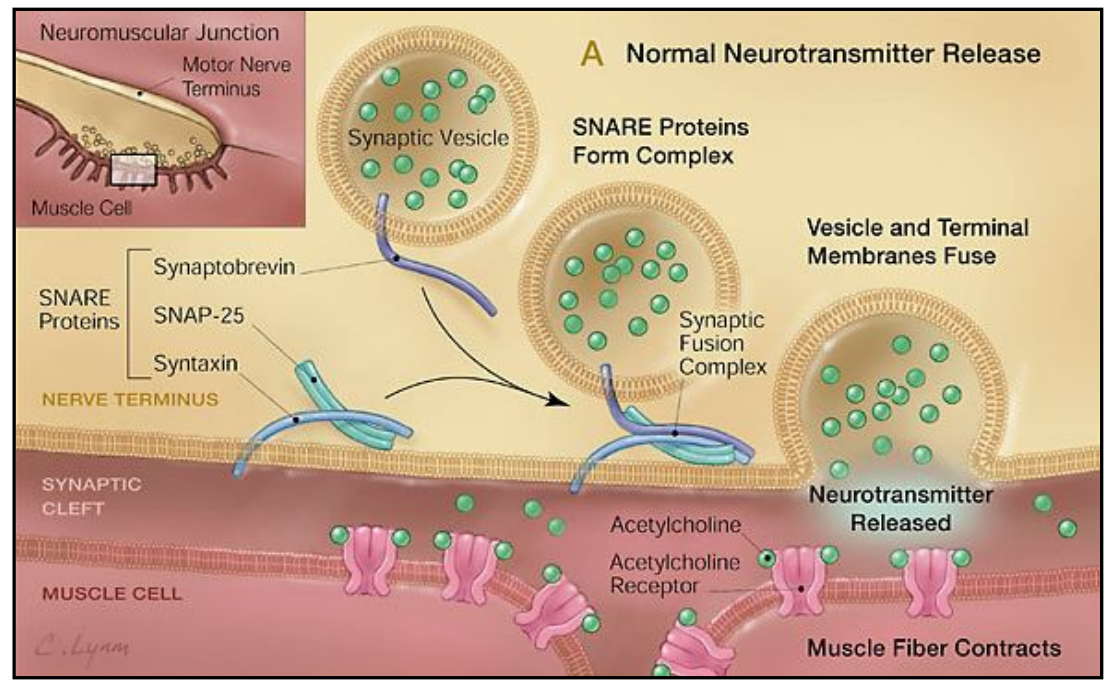

Abb. 7: Physiologische Reizweiterleitung an der cholinergen Synapse:

Die mit ACh gefüllten Endosomen vereinen sich mit dem Exozytoseapparat, bestehend aus Synaptobrevin, SNAP-25 und Syntaxin. So kann ACh in den synaptischen Spalt gelangen und zu den muskarinergen oder nikotinergen Rezeptoren wandern um diese zu aktivieren. (Arnon et al. 2001, S. 1061)

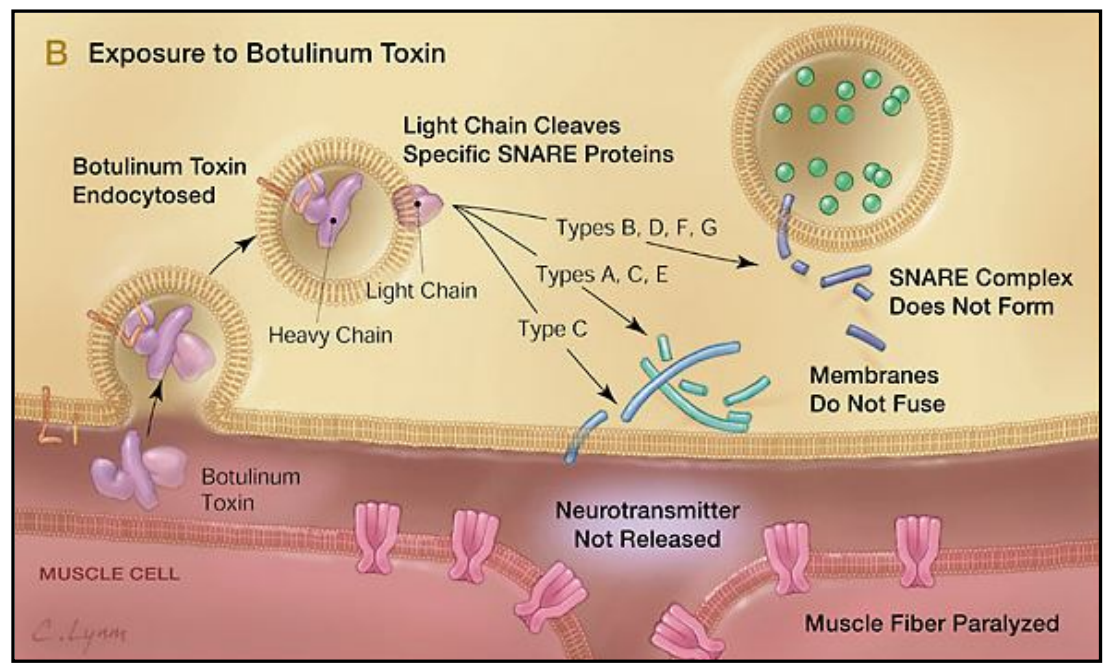

Abb. 8: Wirkmechanismus von Botulinumtoxin an der cholinergen Synapse:

Die schwere Kette (heavy chain) des Botulinumtoxins ermöglicht die rezeptorvermittelte Endozytose in die Zielzelle. Innerhalb der Zelle löst sich die leichte Kette (light chain) von der schweren Kette und kann so Teile des Exozytoseapparates zerstören. Eine Verschmelzung der Endosomen mit dem Exozyoseapparat und somit mit der Zellmembran ist nicht mehr möglich. Eine ACh-vermittelte Reizweiterleitung kann nicht mehr stattfinden. (Arnon et al. 2001, S. 1061)

Botulinumtoxine sind Proteine, die aus einer schweren und einer leichten Kette bestehen, welche über eine Disulfidbrücke miteinander verbunden sind. Beiden 
Ketten werden unterschiedliche Eigenschaften zugeschrieben. Die schwere Kette vermittelt die spezifische, rezeptorvermittelte Endozytose in die cholinerge Nervenzelle. So gelangt der Toxinkomplex in die Endosomen der Nervenzelle. Die leichte Kette zeigt intrazellulär, nach Spaltung der Disulfidbrücke, ihre toxische Wirkung als Protease (Binscheck und Wellhörner 2004). Als spezifische Proteasen spalten die unterschiedlichen Serogruppen verschiedene Komponenten des Exozytoseapparates. Dieser besteht aus unterschiedlichen intrazellulären Proteinen, welche die Voraussetzung zum Verschmelzen der Endosomen mit der präsynaptischen Membran erst ermöglichen. In Bezug auf Botulinumtoxin sind v. a. die Proteine Synaptobrevin, Syntaxin und SNAP-25 („synaptosomal associated protein of 25 kiloDalton") des Exozytoseapparates wichtig. So wird SNAP-25 von Botulinumtoxin $A$ und $E$ gespalten, Synaptobrevin von $B, D, F$ und $G$ zerstört und Syntaxin durch $C$ gebunden (Hayashi et al. 1994). Auf diese Weise werden die Proteine von Botulinumtoxin irreversibel zerstört, so dass es, sowohl an der quergestreiften als auch an der glatten Muskulatur, zu keiner ACh-vermittelten Stimulusübertragung an den cholinergen Synapsen mehr kommen kann (siehe Abb. 8).

\subsubsection{Regeneration der präsynaptischen Nervenzelle}

Obwohl Botulinumtoxin eine irreversible Wirkung am Exozytoseapparat hinterlässt, ist die cholinerge Nervenzelle zu einer Regeneration fähig. Diese erfolgt über zwei verschiedene Wege: einerseits wird das Botulinumtoxin innerhalb des Neurons proteolytisch gespalten und die zerstörten Proteine des Exozytoseapparates werden neu synthetisiert. Andererseits sprossen neue Axone aus, das so genannte „sprouting“. Dabei erfolgt die Resynthese der verschiedenen Komponeneten des Exozytoseapparates in unterschiedlichen Zeitspannen. Diese Erkenntnis erklärt somit die sich unterscheidende Wirkdauer zwischen den verschiedenen Botulinumtoxin-Serogruppen (Breit und Heckmann 2000). 


\subsubsection{Messung der biologischen Aktivität von Botulinumtoxin}

Um Botulinumtoxin an seiner biologischen Aktivität messbar zu machen, werden „Units“ $(U)$ angegeben. Eine $U$ entspricht der letal dose 50, das heißt der letalen Dosis, bei der $50 \%$ der Versuchstiere, Mäuse bei intraperitonealer Applikation, versterben. Diese international anerkannte Dosierungseinheit wurde an SwibbWebster-Mäusen mit einem Gewicht von 18-20g bestimmt (Pütz 2004). Eine Flasche Botox ${ }^{\circledR}$ enthält beispielsweise Trockensubstanz, die $100 \mathrm{U}$ Botulinumtoxin A entsprechen. Diese ist zur Lösung in steriler 0,9\%-Natriumchloridlösung vorgesehen (Rote Liste 2009).

\section{$1.4 \quad$ Fragestellung}

In der Literatur sind bereits mehrfache Erfolge bei der Behandlung von Dysphagien mit Botulinumtoxin A beschrieben. Im Bereich des UES beschreibt die überwiegende Anzahl der Untersuchungen ein kleines Patientenkollektiv mit bis zu zehn Patienten (Blitzer und Brin 1997; Ashan et al. 2000; Dunne et al. 1993; Haapaniemi et al. 2001; Moerman et al. 2002; Restivo et al. 2000; Restivo et al. 2002; Schneider et al. 1994). Vergleichend finden sich im Bereich des LES Untersuchungen, die bis zu 60 Patienten einschließen (Cuillière et al. 1997; Fishman et al. 1996; Prakash et al. 1999).

Botulinumtoxin A wird bei Dysphagiepatienten in der Klinik für Hals-Nasen-Ohrenheilkunde (Direktor: Prof. Dr. Ch. Matthias) wie auch in der Abteilung Gastroenterologie und Endokrinologie (Direktor: Prof. Dr. Dr. h.c. G. Ramadori) der Universitätsmedizin Göttingen therapeutisch eingesetzt.

Ziel der vorliegenden Analyse ist die retrospektive, klinische Auswertung der mit Botulinumtoxin A behandelten Patienten, welche unter einer Dysphagie litten. Bei dieser Auswertung werden folgende Parameter besonders berücksichtigt:

- Symptomatik,

- Begleiterkrankungen,

- Dauermedikation, 
- Beschwerdedauer,

- Dosierung,

- Ansprechen auf diese Therapie,

- Wirkung,

- Wirkungseintritt,

- Dauer der Wirkung,

- unerwünschten Nebenwirkungen.

Weiterhin soll eine vergleichende Darstellung alternativer Therapiemöglichkeiten im Hinblick auf Ansprechen, Wirkdauer, Invasivität und auf unerwünschte Nebenwirkungen erfolgen. 


\section{MATERIAL und METHODEN}

\subsection{Patientenkollektiv}

Die vorliegende retrospektive, klinische Analyse wurde an 48 Patienten mit einem Mindestalter von 18 Jahren und gesicherter Dysphagie aufgrund einer Motilitätsstörung im Bereich des UES oder des LES durchgeführt. Es wurden Patienten, die sich zwischen Februar 1996 und April 2007 im Universitätsklinikum Göttingen am Zentrum für Innere Medizin in der Abteilung für Gastroenterologie und Endokrinologie (Direktor: Prof. Dr. Dr. h.c. G. Ramadori) oder zwischen Januar 2004 und September 2008 in der Klinik für Hals-Nasen-Ohrenheilkunde (Direktor: Prof. Dr. Ch. Matthias) behandeln ließen, in die Analyse aufgenommen. Behandelnde Ärzte in der Abteilung Gastroenterologie und Endokrinologie waren Prof. Dr. H. Schwörer bzw. Prof. Dr. R. Laskawi in der Klinik für Hals-Nasen-Ohrenheilkunde.

Patienten, die Kontraindikationen zur Botulinumtoxin-A-Therapie aufwiesen, wie z. B. Myasthenia gravis, Lambert-Eaton-Syndrom, eine bekannte Überempfindlichkeit gegen Botulinumtoxin A zeigten, sowie Schwangere bzw. stillende Mütter, die Unterschreitung des Mindestalters, sowie eine nicht muskuläre Ursache für die Dysphagie, wurden nicht therapiert.

Das diagnostische und therapeutische Management beinhaltete die persönliche Vorstellung der Patienten, ausführliche Anamnese, klinische Untersuchung, sowie weitere diagnostische Maßnahmen zur Charakterisierung der Dysphagie. Die Diagnostik wurde mittels Videofluoroskopie, Ösophagusbreischluck, Magnetresonanztomographie (MRT), CT, Röntgen-Thorax, Ösophagogastroduodenoskopie, Endosonographie, Manometrie und/oder pH-Metrie durchgeführt. Dieses diagnostische Spektrum wurde entsprechend der Anamnese und klinischen Untersuchung jedes Patienten individuell angepasst, so dass nicht jeder Patient die gesamte diagnostische Breite dieser Untersuchungsmethoden erfuhr.

Abgesehen von der Indikationsstellung zur Botulinumtoxintherapie wurden die Patienten vor der ersten Injektion über den „off-label-use“ von Botulinumtoxinprä- 
paraten ausführlich aufgeklärt. Die Aufklärung ist im HNO-ärztlichen Bereich erfolgte mit einer speziell angefertigten schriftlichen Einverständniserklärung (siehe Anhang S. 73-74). Die Aufklärung zur Botulinumtoxin-A-Injektion im gastroenterologischen Bereich erfolgte mittels Aufklärungsblatt für die Ösophagogastroduodenoskopie, auf dem handschriftlich die folgenden Punkte eingetragen wurden, die bei der Aufklärung speziell angesprochen und erläutert wurden:

- Injektion von Botulinumtoxin A im Bereich des LES am Übergang von Speiseröhre und Magen.

- Komplikationen und unerwünschte Wirkungen von Botulinumtoxin A:

- Organverletzungen jeglicher Art,

- Schmerzen an der Injektionsstelle und Epiglottis,

- Lungenentzündung und Infektionen,

- Blutungen und Blutergüsse (Hämatome),

- Schluckbeschwerden,

- Reflux, eventuell mit Sodbrennen verbunden,

- Lähmung des Zwerchfells, gegebenenfalls mit temporärer künstlicher Beatmung auf Intensivstation.

- alternative Behandlungsmethoden mit Indikation, sowie Vor- und Nachteile der einzelnen Behandlungsmethoden (medikamentöse Therapie, pneumatische Dilatation, operative Verfahren).

\subsection{Therapie mit Botulinumtoxin}

\subsubsection{Behandlung im Bereich des UES (oberer Ösophagussphinkter)}

Patienten mit einer Motilitätsstörung im Bereich des UES wurden stationär aufgenommen. Die Eingriffe wurden in Intubationsnarkose durchgeführt. Vorbereitend wurden einer handelsüblichen Butterfly-Schlauchkanüle die beiden „Halteflügel“ abgeschnitten und die Nadel sowie der Schlauch an einem Zangeninstrument mittels Klebeverband längs befestigt (siehe Abb. 9). 


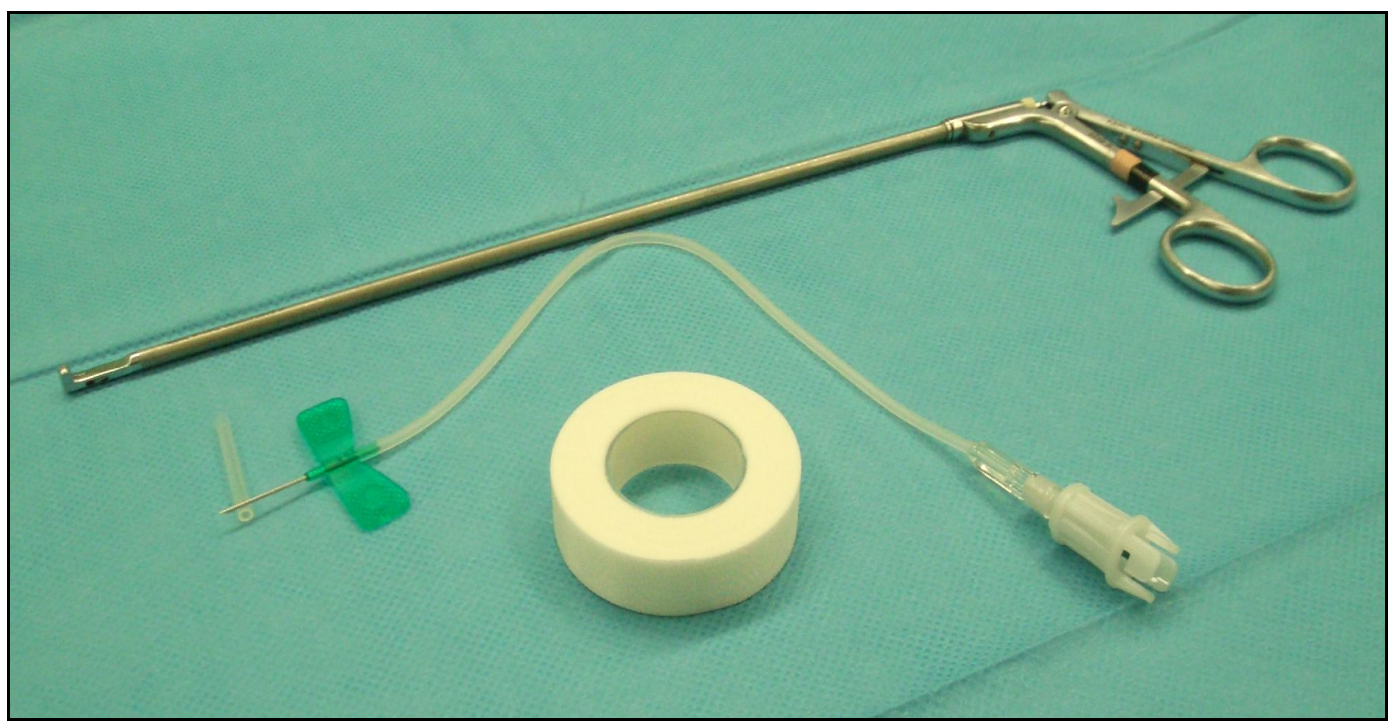

Abb. 9: Zur Applikation von Botulinumtoxin benötigte Materialien:

- arretierbares Zangeninstrument,

- handelsübliche Butterfly-Schlauchkanüle,

- Klebeverband zur Befestigung.

Das Laryngoskopierohr wurde transoral eingeführt. Nach Darstellung des UES erfolgte die intramuskuläre Botulinumtoxin-A-Injektion unter mikroskopischer Kontrolle (siehe Abb. 10 und 11).

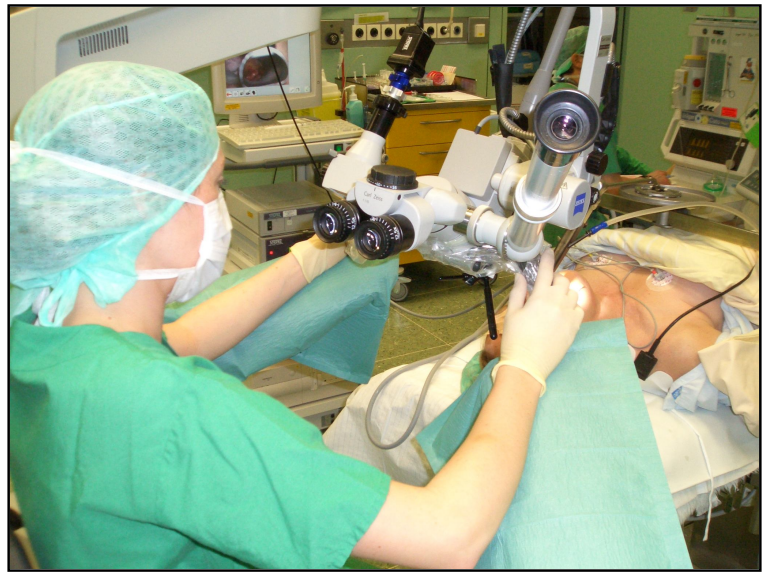

Abb. 10: Mikrolaryngoskopie intraoperativ:

Transnasaler Zugang in starrer Endoskopie mit Mikroskop

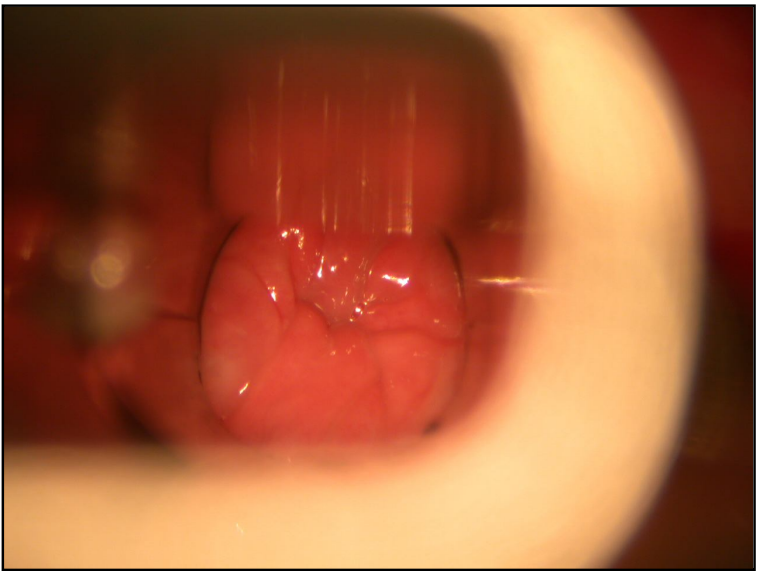

Abb. 11: Mikroskopische Sicht auf den UES:

Rosettenartiger Sphinkter in Kontraktion

Zur einheitlichen Konzentration wurden $100 \mathrm{U}$ Botulinumtoxin A in 4 Milliliter (ml) physikalischer 0,9\%-haltiger Natriumchloridlösung gelöst, so dass in $1 \mathrm{ml}$ Lösung $25 \mathrm{U}$ enthalten waren. Die Gesamtdosierung wurde zwischen $30 \mathrm{U}$ und $75 \mathrm{U}$ ap- 
pliziert und in die beiden seitlichen und die hintere Circumferenzen, insgesamt drei Punkte, injiziert. Patienten mit einer funktionellen Hypersalivation erhielten teilweise darüber hinaus Botulinumtoxininjektionen in die Glandulae parotideae und die Glandulae submandibulares. Patienten mit einer vorbestehenden oromandibulären Dystonie erhielten optional weitere Botulinumtoxininjektionen in den Bereich des Mundbodens, des Platysmas und perioral.

\subsubsection{Behandlung im Bereich des LES (unterer Ösophagussphinkter)}

Patienten mit einer Motilitätsstörung im Bereich des LES wurden entweder ambulant behandelt oder stationär aufgenommen. Gründe für eine stationäre Aufnahme waren erstmalige Behandlung mit Botulinumtoxin oder hohes Lebensalter mit kardiovaskulären oder respiratorischen Begleiterkrankungen. Im Anschluss an die Behandlung mit Botulinumtoxin A wurden alle Patienten herz- und kreislaufüberwacht. Patienten mit ambulanter Behandlung wurden über die Verkehrsuntauglichkeit aufgeklärt und zu Verlaufskontrollen erneut ambulant einbestellt. Die endoskopische Untersuchung fand jeweils in Linksseitenlage unter Herzkreislaufüberwachung mittels Blutdruck- und Herzfrequenzmessung, Elektrokardiogramm (EKG)-Ableitung und Messung der Sauerstoffsättigung statt. Bei allen Patienten war ein peripherer venöser Zugang vorhanden. Nach lokaler Anästhesie der Rachenhinterwand wurde das Endoskop vorsichtig unter Sicht eingeführt. Sobald das Endoskop sicher im Ösophagus platziert war und keine Aspirationsgefahr bestand, wurden die Patienten mit Midazolam und Propofol sediert. Diese Vorgehensweise wurde gewählt, damit keine Aspiration von eventuell in der Speiseröhre vorhandenem unverdautem Nahrungsbrei erfolgen konnte. Unter prograder Sicht wurde der Bereich des LES mittels Ösophagogastroduodenoskopie eingestellt (siehe Abb. 12 und 13). Dabei konnte über eine handelsübliche sieben Millimeter Sklerosierungsnadel eine Bolusapplikation von 3-6 ml Glyceroltrinitrat des Präparats Nitrolingual ${ }^{\circledR}$ infus. (G. Pohl-Boskamp, Hohenlockstedt, Deutschland) in einer Lösung von 0,5 $\mathrm{mg}$ in $1 \mathrm{ml}$ in die LES-Region erfolgen. Mit Hilfe dieser Methode konnte bei drei Patienten eine Relaxation des LES erreicht werden. Im Anschluß wurde über den gleichen Zugangsweg die Botulinumtoxininjektionen vorgenommen. 


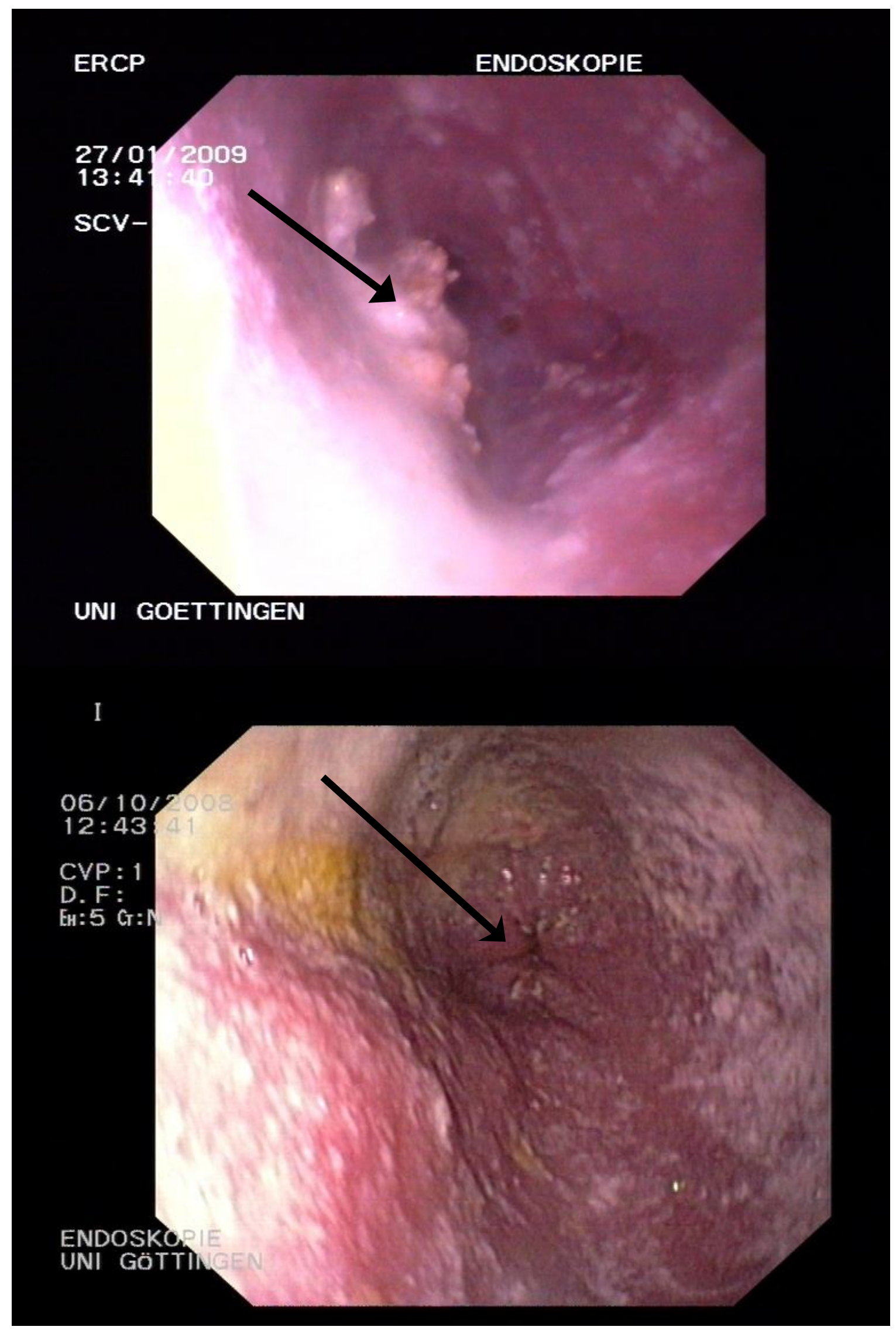

Abb. 12 und 13: Endoskopische Sicht auf den distalen Ösophagus Oberes Bild: Ösophagus mit $\rightarrow$ : verbliebenen Speiseresten. Unteres Bild: $\rightarrow$ : rosettenartige Kontraktion des distalen Ösophagus. 
Auch hier wurde eine einheitliche Lösung von $100 \mathrm{U}$ Botulinumtoxin A (gelöst in 5 $\mathrm{ml}$ 0,9\%-haltiger Natriumchloridlösung) gewählt, so dass in $1 \mathrm{ml} 20 \mathrm{U}$ Botulinumtoxin A gelöst waren. Es wurde in der sogenannten Vier-Quadrantentechnik gearbeitet, bei der Botulinumtoxin A in $1 \mathrm{ml}$ Portionen injiziert wird. Die Injektionspunkte wurden nach der Uhr bei 12, 3, 6 und 9 Uhr gesetzt. In der Regel wurden insgesamt $100 \mathrm{U}$ verabreicht, so dass der verbliebene $1 \mathrm{ml}$ je zur Hälfte zwischen 3 und 6 Uhr bzw. zwischen 9 und 12 Uhr in den Bereich des LES appliziert wurde. Im Anschluss an die Injektionen wurde das Endoskop in den Magen vorgeschoben und dort drei bis fünf Minuten belassen, um eine zusätzliche Dehnung des LES zu erzielen. Bei einem Patienten wurde eine gleichzeitige pneumatische Dilatation vorgenommen.

\subsection{Datenerhebung}

Die notwendigen Daten für die vorliegende Untersuchung wurden anhand der archivierten Patientendaten sowie telefonischer Rücksprachen gewonnen. Der Erfolg der Therapie wurde über die subjektiven Aussagen der Patienten selbst bzw. durch deren Eltern, Kinder oder Pflegepersonal bestimmt. Es wurde erfragt, ob eine Verbesserung der Dysphagie, inklusive der Möglichkeit von fester und/oder flüssiger Nahrungsaufnahme, symptomatisch eintrat. Die so gewonnenen Daten wurden teilweise durch nachfolgende Diagnostik, wie Videofluoroskopie, neurofunktionelle Schluckaktanalyse, Ösophagogastroduodenoskopie, Ösophagusbreischluck, Manometrie oder CT objektiviert. Durch den teilweise deutlich reduzierten Allgemeinzustand der Patienten war jedoch nicht in allen Fällen eine apparative diagnostische Kontrolle durchführbar.

Neben der subjektiven Wirkung der Therapie wurden weitere Daten erhoben. Auf diese Weise wurden Wirkungseintritt und -dauer, unerwünschte Nebenwirkungen, Injektionen und Dosierungen, vorangegangene Therapien, Beschwerdedauer, Symptomatik und Hauptdiagnosen ermittelt und bei der Interpretation der Ergebnisse berücksichtigt. Ebenso wurden wiederholte Sitzungen beachtet und ins Verhältnis zu der initialen Injektion gesetzt, unter Beachtung der Dosierung und des Intervalls zwischen den Behandlungen. 
Die extrahierten Daten wurden mit Hilfe des Computerprogramms Microsoft Excel( $)$ digital zusammengetragen, ausgewertet und in Grafiken und Tab. optisch dargestellt. 


\section{ERGEBNISSE}

\subsection{Charakterisierung des Patientenkollektivs}

Es stellten sich insgesamt 48 Patienten mit einer gravierenden Dysphagie und dem Wunsch der Botulinumtoxinapplikation im Universitätsklinikum Göttingen vor. Das Patientenkollektiv setzte sich aus 33 Männern und 15 Frauen zusammen, mit einer Altersverteilung von 25 bis 85 Jahren. In Anbetracht der Botulinumtoxinapplikation im oberen oder unteren Ösophagussphinkter wurden die Patienten entsprechend ihrer Dysphagie in zwei Gruppen aufgeteilt: 17 Patienten wurden der Gruppe „Oberer Ösophagussphinkter“ (UES) und 31 Patienten der Gruppe „Unterer Ösophagussphinkter“ (LES) zugeordnet. Eine Übersicht über das Patientenkollektiv ist Tab. 2 zu entnehmen.

Tab. 2: Charakteristika des Patientenkollektivs mit unterschiedlicher Dysphagie

\begin{tabular}{|l|r|r|r|r|r|r|r|r|}
\hline & UES & neurogen & Dystonie & Tumor & $\begin{array}{c}\text { unklare } \\
\text { Genese }\end{array}$ & LES & Gesamt \\
\hline Anzahl der Patienten & 17 & 7 & 5 & 3 & 2 & 31 & $\mathbf{4 8}$ \\
\hline minimales Alter in Jahren & 25 & 59 & 25 & 42 & 55 & 25 & $\mathbf{2 5}$ \\
\hline maximales Alter in Jahren & 80 & 80 & 75 & 74 & 59 & 85 & $\mathbf{8 5}$ \\
\hline Alter in Jahren als Median & 64 & 69 & 64 & 64 & 57 & 57 & 64 \\
\hline Verhältnis Mann:Frau & $13: 4$ & $7: 0$ & $1: 4$ & $3: 0$ & $2: 0$ & $20: 11$ & $\mathbf{3 3 : 1 5}$ \\
\hline Beschwerdedauer in Monaten als Median & 6,5 & $0-3$ & $>24$ & $4-6$ & $>24$ & $>24$ & $>\mathbf{2 4}$ \\
\hline
\end{tabular}

\subsubsection{Gruppe UES (oberer Ösophagussphinkter)}

Durch die unterschiedliche Genese der Dysphagie konnten die Patienten der Gruppe UES $(n=17)$ bezüglich ihrer Hauptdiagnose nochmals in vier weitere Untergruppen unterteilt werden. Die erste Hauptdiagnose war "neurogenen" Ursprungs $(n=7)$, d. h. sie liegt im Bereich des zentralen Nervensystem aufgrund cerebraler Ischämien bei Zustand nach (Z. n.) Apoplex oder Reanimation. Es wurde bei $43 \%(n=3)$ der Patienten in dieser Untergruppe $(n=7)$ ein WallenbergSyndrom diagnostiziert. Das Wallenberg-Syndrom wird zu den Hirnstammsyndromen gezählt und ist auf eine Schädigung im Bereich der dorsolateralen Medulla oblongata zurückzuführen (Gleixner et al. 2004/05). Die zweite Hauptdiagnose 
bezog sich auf eine ursächliche „Dystonie” im oromandibulären Bereich $(n=5)$. Auf „Tumore" im Larynxbereich und v. a. auf die Folgen ihrer vorangegangenen Therapien, wie eine Laryngektomie, eine Radio- und Chemotherapie, bezog sich die dritte Hauptdiagnose $(n=3)$. In der vierten Untergruppe waren Patienten mit einer Dyskinesie des UES „unklarer Genese“ zusammengefasst $(n=2)$.

\subsubsection{Gruppe LES (unterer Ösophagussphinkter)}

Bei Patienten der Gruppe LES ( $n=31$ ) wurde bei 90,3\% ( $n=28$ ) die Hauptdiagnose "Achalasie" gestellt. Bei den übrigen 9,7\% ( $n=3)$ der Patienten stellte sich ein „Nussknackerösophagus" oder ein „diffuser Ösophagusspasmus“ manometrisch und radiologisch dar.

\subsection{Begleiterkrankungen und Medikation des Patienten- kollektivs}

\subsubsection{Begleiterkrankungen des Patientenkollektivs}

Neben der Dysphagie litten insgesamt 72,9\% $(n=35)$ des gesamten Patientenkollektivs $(n=48)$ an weiteren Erkrankungen. Teilweise waren die Patienten an mehr als einer Krankheit erkrankt, so dass im Folgenden Mehrfachnennungen möglich sind. Dabei wurden v. a. kardiovaskuläre Erkrankungen, wie beispielsweise arterielle Hypertonie, koronare Herzkrankheit (KHK) oder Hirninfarkt beschrieben. Auch tumoröse Geschehen, beispielsweise Larynxkarzinom, Mammakarzinom und Prostatakarzinom, oder ein Z. n. aktueller Operation in den letzten sechs Monaten zählten zu den häufigsten Erkrankungen. Eine Übersicht über die Verteilung der Begleiterkrankungen des Patientenkollektivs liefert Abb. 14. 


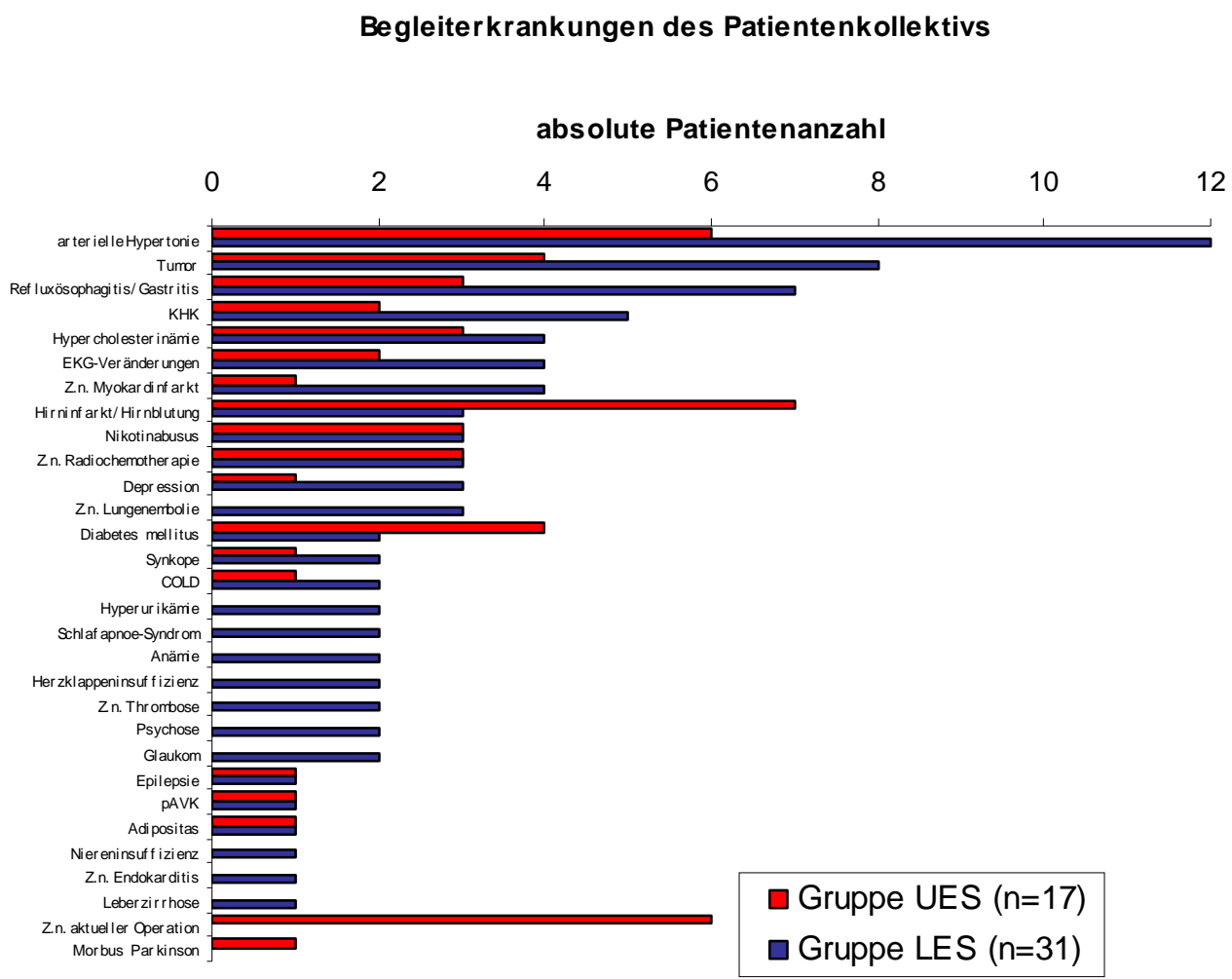

Abb. 14: Begleiterkrankungen des Patientenkollektivs für die jeweiligen Gruppen UES und LES

\subsubsection{Gruppe UES (oberer Ösophagussphinkter)}

Patienten der Gruppe UES ( $n=17)$ litten zu 76,5\% ( $n=13)$ unter weiteren Erkrankungen. Dabei gaben $41,2 \%(n=7)$ der gesamten Gruppe eine Hirnblutung bzw. einen Hirninfarkt anamnestisch an. 35,3\% $(n=6)$ litten unter arterieller Hypertonie oder hatten eine Operation in den letzten sechs Monaten. Von einem Tumorleiden, Z. n. Larynx- oder Hypopharynxkarzinom und Mammakarzinom, oder einem Diabetes mellitus berichteten $23,5 \%(n=4)$ der Patienten. 17,6\% $(n=3)$ der Patienten klagten über eine Refluxösophagitis bzw. einer Gastritis, litten an Hypercholesterinämie, wurden bereits mit einer Radiochemotherapie behandelt oder konsumierten regelmäßig Nikotin. An einer KHK oder an EKG-Veränderungen, wie beispielsweise Rhythmusstörungen und Blockbilder, litten 11,8\% ( $n=2)$ der Patienten. Jeweils 5,9\% $(n=1)$ der Patienten hatten einen Myokardinfarkt oder eine Synkope in der Vorgeschichte, litten an Depression, chronisch-obstruktiver Lungenerkrankung (COLD), peripher-arterieller Verschlusskrankheit (pAVK), Adipositas, Epilepsie oder Morbus Parkinson (siehe Tab.14). 


\subsubsection{Gruppe LES (unterer Ösophagussphinkter)}

Bei Patienten der Gruppe LES ( $n=31)$ fanden sich in 71,0\% $(n=22)$ der Fälle eine oder mehrere weitere Erkrankungen. Insgesamt 38,7\% $(n=12)$ berichteten von einem arteriellen Hypertonus und 25,8\% $(n=8)$ von einem Tumorleiden, wie $z$. B. Hauttumore (Melanom und Basaliom), supraglottisches Karzinom, Colonkarzinom, Bronchialkarzinom, Prostatakarzinom oder Antrumkarzinom. Weitere 22,6\% ( $n=7)$ litten an einer Refluxösophagitis bzw. einer Gastritis und 16,1\% $(n=5)$ an einer KHK. Wiederum je 12,9\% ( $n=4)$ zeigten Hypercholesterinämie, EKG-Veränderungen bzw. einen Myokardinfarkt in der Vorgeschichte. Über eine Hirnblutung bzw. einen Hirninfarkt, Radiochemotherapie oder Lungenembolie in der Vorgeschichte oder über Depression und Nikotinabusus berichteten jeweils 9,7\% $(n=3)$ des Patientenkollektivs. Bei jeweils 6,5\% $(n=2)$ der Patienten der Gruppe LES fand sich eine Thrombose, Synkope, COLD, Diabetes mellitus, Hyperurikämie, Schlafapnoe-Syndrom, Anämie, Herzklappeninsuffizienz, Psychose oder ein Glaukom in der Anamnese. An Epilepsie, pAVK, Adipositas, Niereninsuffizienz, Leberzirrhose, sowie einer stattgehabten Endokarditis litten 3,2\% $(n=1)$ der Patienten. Die Daten sind ebenfalls in Abb. 14 zusammengefasst.

\subsubsection{Medikation des Patientenkollektivs}

Neben den Begleiterkrankungen wurde die Dauermedikation des Patientenkollektivs $(n=48)$ beachtet. Mehrfachnennungen waren hierbei ebenfalls möglich. Eine Übersicht über die Verteilung der Dauermedikation des gesamten Patientenkollektivs liefert Abb. 15.

Insgesamt 87,5\% ( $n=42)$ nahmen täglich mindestens ein Medikament ein. Die Medikation wurde nach der Wirkstoffgruppe zusammengefasst. Häufige Medikamente waren Protonenpumpeninhibitoren (PPI), wie beispielsweise Pantoprazol, und Antikoagulanzien, beispielsweise Acetyl-Salicylat, Clopidogrel, Heparine und Phenprocoumon. 


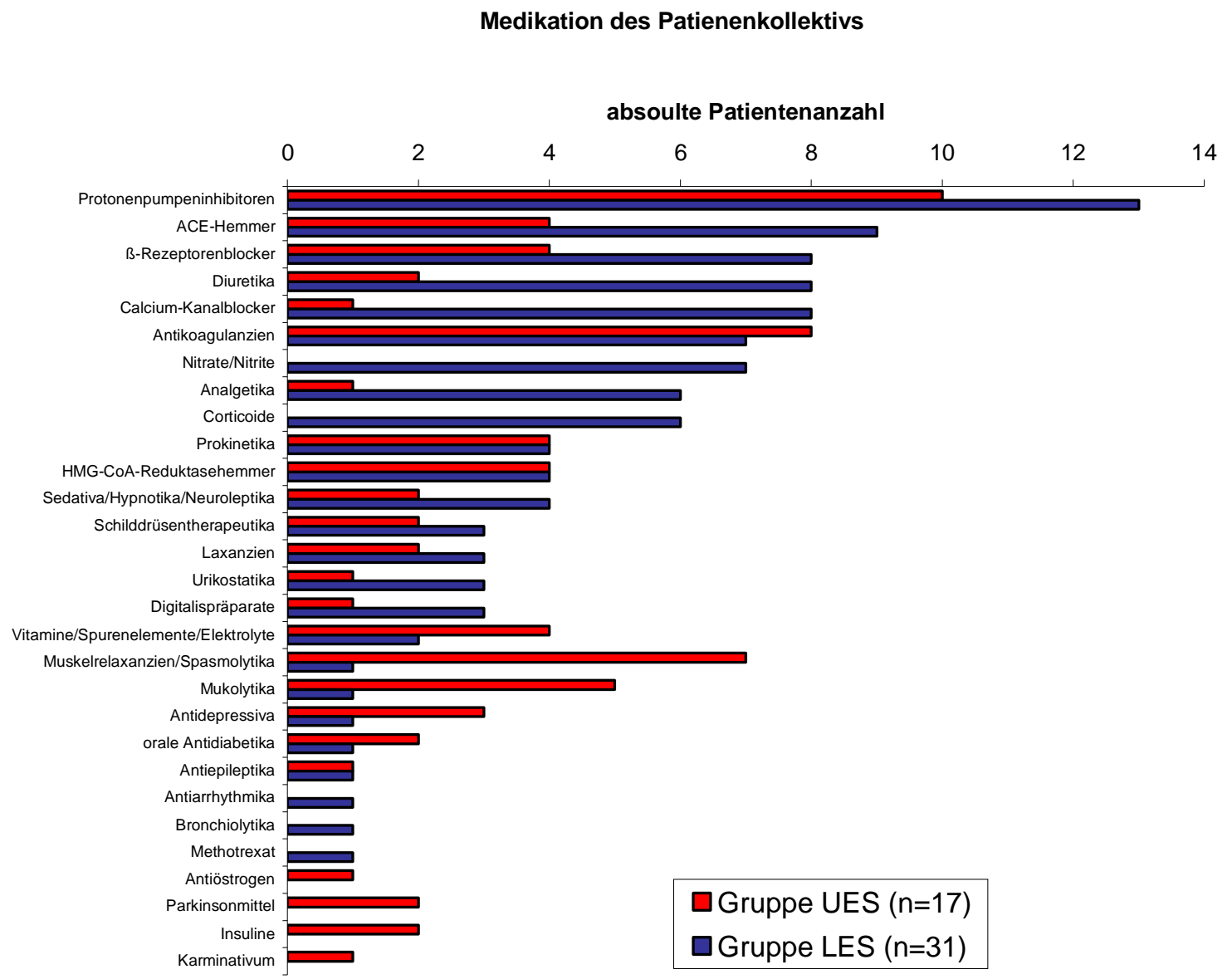

Abb. 15: Medikation des Patientenkollektivs für die jeweiligen Gruppen UES und LES

\subsubsection{Gruppe UES (oberer Ösophagussphinkter)}

Insgesamt 94,1\% ( $n=16)$ der Patienten der Gruppe UES ( $n=17)$ wiesen eine Dauermedikation auf. Einen PPI nahmen 58,8\% $(n=10)$ der Patienten ein und $47,1 \%(n=8)$ standen unter Antikoagulation. Weitere $41,2 \%(n=7)$ nahmen ein Muskelrelaxanz bzw. ein Spasmolytikum, wie z. B. Trihexyphenidyl, Baclofen und Tizanidin, und 29,4\% ( $n=5)$ ein Mukolytikum, z. B. Acetylcystein, ein. ACE-Hemmer, z. B. Lisinopril bzw. Captopril, B-Rezeptorenblocker, z. B. Metoprolol, Prokinetika, z. B. Metoclopramid, 3-Hydroxy-3-methylglutaryl-CoAReduktasehemmer (HMG-CoA-Reduktasehemmer), wie z. B. Atorvastatin, und Vitamine, Spurenelemente und Elektrolyte, z. B. B-Vitamine bzw. Magnesium, wurden zu jeweils $23,5 \%(n=4)$ eingenommen. In 17,6\% ( $n=3)$ wurden Antidepressiva, wie z. B. Citalopram bzw. Mirtazapin, eingenommen. Zu jeweils $11,8 \%(n=2)$ wurden Diuretika, z. B. Torasemid und Furosemid, Sedativa, Hypnotika und Neuroleptika, z. B. Clonazepam bzw. Prothipendyl, Schilddrüsentherapeutika, z. 
B. Levothyroxin, Laxanzien, z. B. Lactulose, orale Antidiabetika, z. B. Acarbose, Parkinsonmittel, z. B. Levodopa, und Insuline verabreicht. Karminativa, z. B. Simeticon, Antiepileptika, z. B. Gabapentin, Digitalispräparate, z. B. BAcetyldigoxin, Urikostatika, z. B. Allopurinol, Analgetika, z. B. Meloxicam, Antiöstrogene, z. B. Tamoxifen, und Calcium-Kanalblocker, z. B. Nifedipin, wurden zu jeweils 5,9\% ( $n=1)$ eingenommen (siehe Abb. 15).

\subsubsection{Gruppe LES (unterer Ösophagussphinkter)}

Patienten dieser Gruppe $(n=31)$ standen in 83,9\% $(n=26)$ unter Dauermedikation. 41,9\% ( $n=13)$ der Patienten nahmen einen PPI und 29,0\% ( $n=9)$ einen ACE-Hemmer ein. Zu jeweils 25,8\% ( $n=8)$ wurden B-Rezeptorenblocker, Diuretika und Calcium-Kanalblocker eingenommen. In weiteren $22,6 \%(n=7)$ wurden jeweils Antikoagulanzien und Nitrate bzw. Nitrite, wie z. B. Isosorbiddinitrat, und in je 19,4\% $(n=6)$ der Patienten Corticoide, z. B. Prednison bzw. Budesonid, und Analgetika, z. B. Metamizol bzw. Fentanyl, verordnet. Prokinetika, HMG-CoA-Reduktasehemmer und Neuroleptika, Hypnotika und Sedativa, z. B. Olanzapin, wurden in jeweils $12,9 \%(n=4)$ eingenommen. In jeweils $9,7 \%(n=3)$ wurden Schilddrüsentherapeutika, Laxanzien, Urikostatika und Digitalispräparate verordnet. Vitamine, Spurenelemente und Elektrolyte erhielten 6,5\% ( $n=2)$ der Patienten. Zu jeweils 3,2\% $(n=1)$ bei Patienten der Gruppe LES wurden Muskelrelaxanzien bzw. Spasmolytika, Mukolytika, Antidepressiva, orale Antidiabetika, Antiepileptika, Antiarrhythmika, z. B. Sotalol, Bronchiolytika, z. B. Ipratropiumbromid, und Methotrexat eingenommen (siehe Abb. 15).

\subsection{Symptomatik der Patienten}

Die Symptomatik zeigte sich neben der bei fast allen Patienten vorhandenen Dysphagie sehr vielgestaltig und ist für beide Hauptgruppen dargestellt (siehe Abb. 16 und Abb. 17). Patienten klagten teilweise über mehr als ein Symptom, so dass hier Mehrfachnennungen möglich sind. Für die Indikation zur Therapie mit Botulinumtoxin A war bei allen Patienten die Existenz einer Motilitätsabweichung der Muskulatur im Bereich des UES oder des LES relevant. 


\subsubsection{Gruppe UES (oberer Ösophagussphinkter)}

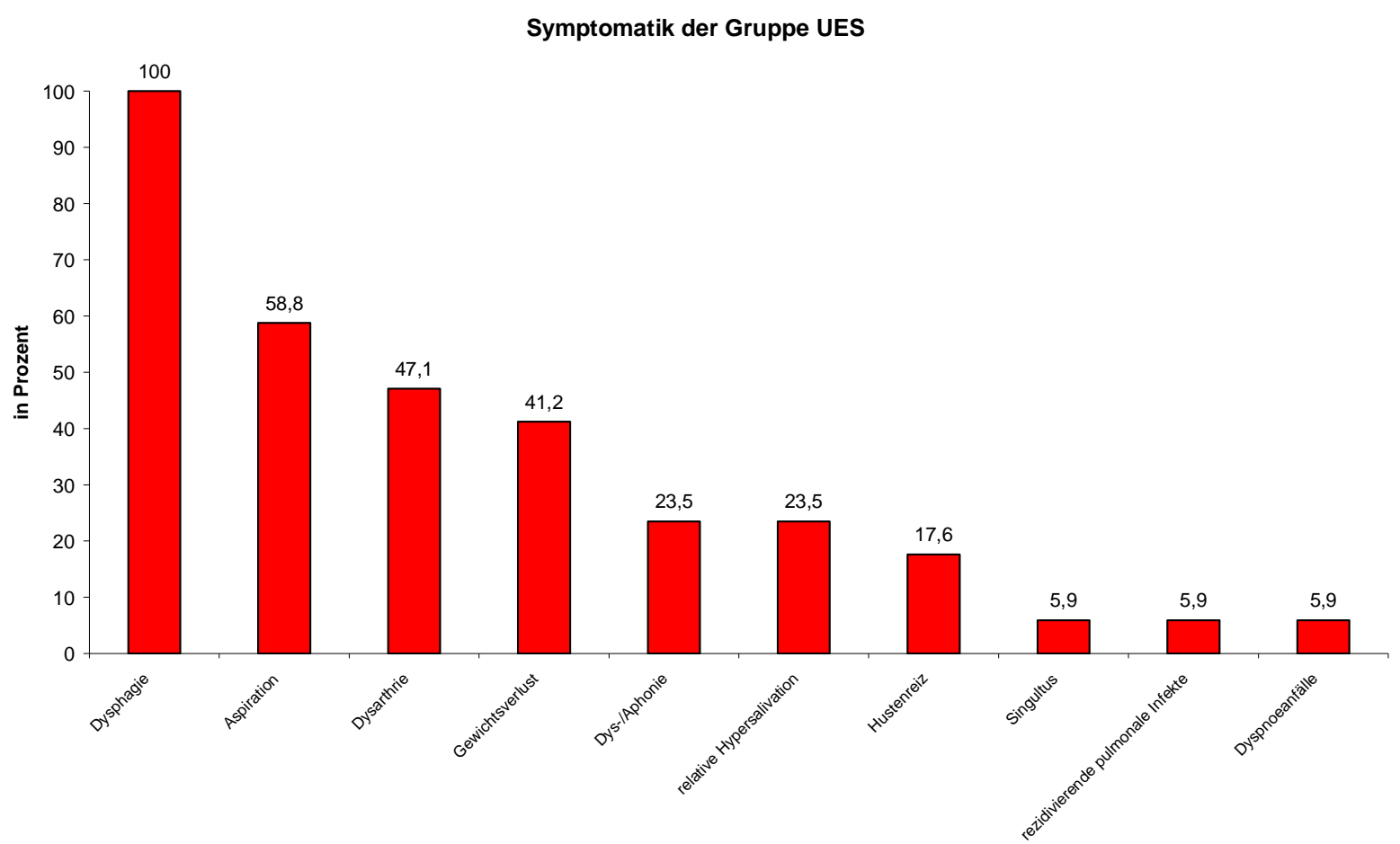

Abb. 16: Verteilung der Symptome in der Gruppe UES $(n=17)$.

Patienten der Gruppe UES ( $n=17)$ litten in 100\% der Fälle unter Dysphagie. Dabei beschrieben die Patienten ihre Dysphagie sehr unterschiedlich. In 70,6\% der Fällen ( $n=12)$ konnten sie weder feste noch flüssige Nahrung zu sich nehmen, da der obere Ösophagussphinkter sich nicht adäquat öffnete. Es kam zur Aspiration und die Ernährung wurde über eine PEG-Sonde sichergestellt. 11,8\% $(n=2)$ berichteten über eine Dysphagie für ausschließlich feste Speisen, so dass sie sich über weiche und flüssige Nahrung ernährten. Weitere 17,6\% $(n=3)$ der Patienten, v. a. die mit einer Dystonie als Grunderkrankung, mussten "den richtigen Zeitpunkt" zum Schlucken abwarten, in der die Tonussteigerung der Muskulatur möglichst gering war, so dass in dieser Zeit die Nahrungsaufnahme unkompliziert erfolgen konnte. Allerdings konnten sie weder feste noch flüssige Kost zu sich nehmen, wenn sie den beschriebenen Zeitpunkt verpasst hatten. 11,8\% ( $n=2)$ der Patienten beschrieben eine kombinierte Problematik mit Dysphagie und Aphonie nach Provoxstimmprothesenanlage bei Z. n. Laryngektomie. 
Bei 58,8\% ( $n=10)$ der Patienten ( $n=17)$ kam es zur Aspiration, 47,1\% $(n=8)$ klagten über Dysarthrie und 41,2\% ( $n=7)$ über Gewichtsverlust. In 23,5\% $(n=4)$ der Fälle beschrieben die Patienten Dys- bzw. Aphonie und relative Hypersalivation und in 17,6\% ( $n=3)$ einen Hustenreiz. Bei 5,9\% ( $n=1)$ der Fälle zeigten sich Singultus, rezidivierende pulmonale Infekte oder Dyspnoeanfälle (siehe Abb. 16).

\subsubsection{Gruppe LES (unterer Ösophagussphinkter)}

Symptomatik der Gruppe LES

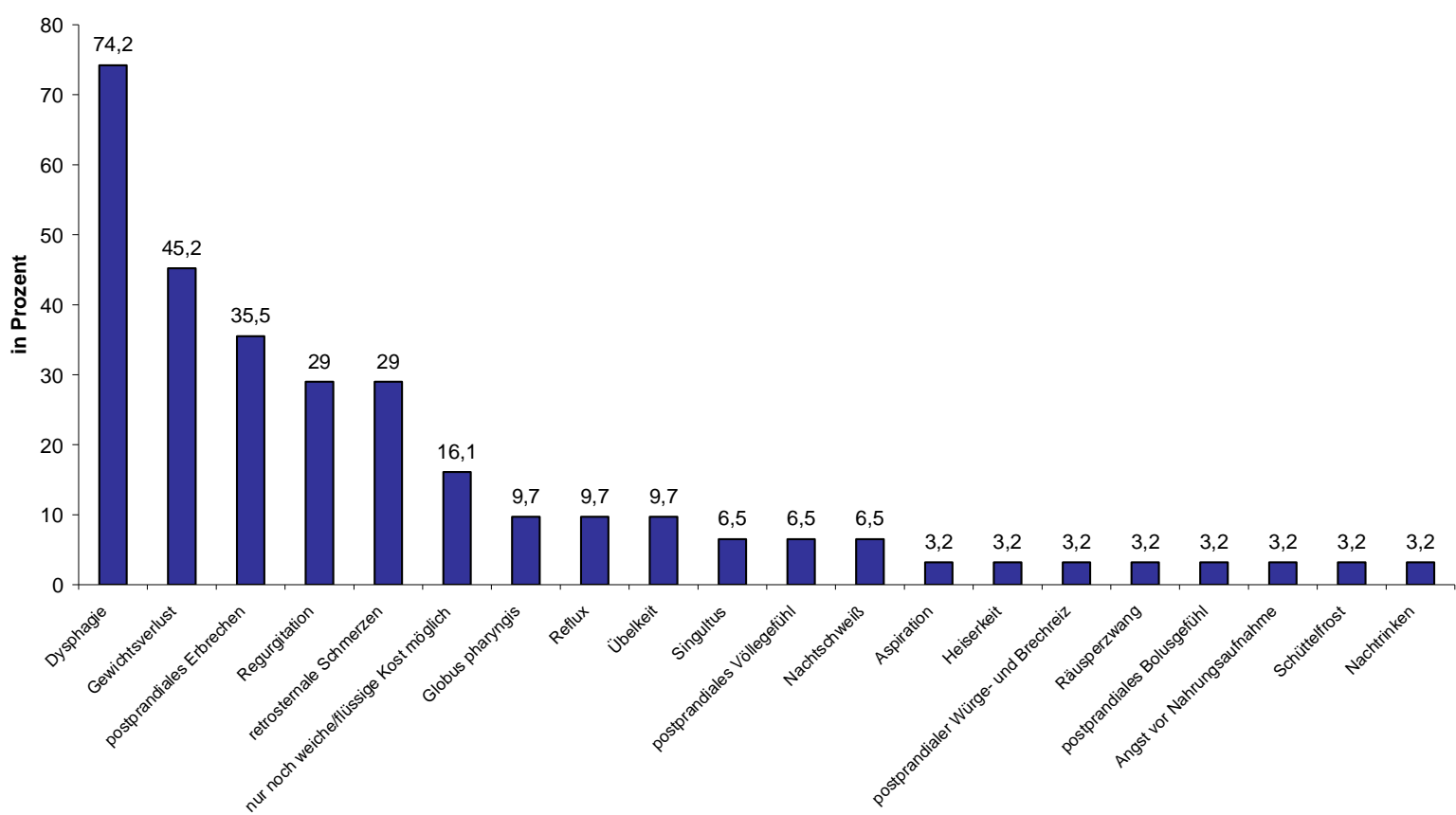

Abb. 17: Verteilung der Symptome in der Gruppe LES ( $n=31)$.

Patienten der Gruppe LES ( $n=31)$ litten in 74,2\% $(n=23)$ der Fälle an Dysphagie (siehe Abb. 17). Auch in dieser Gruppe zeigten sich unterschiedliche Varianten einer Dysphagie. Patienten mit einer fortgeschrittenen Achalasie klagten über Schluckstörungen sowohl für feste als auch für flüssige Nahrung. Teilweise konnten sie noch flüssige Nahrung mit Hilfe von verschiedenen Manövern, wie Essen im Stehen, Strecken des Halses, nach Hintenziehen der Schultern, Nachtrinken und Trinken von stark kohlensäurehaltigen Getränken, zu sich nehmen. Andere Patienten haben sich ausschließlich von flüssiger Kost ernährt, da diese noch „durchrutschen“ konnte. 
45,2\% ( $n=14)$ der Patienten berichteten von einer Gewichtsabnahme, 35,5\% $(n=11)$ litten unter postprandialem Erbrechen und 29,0\% ( $n=9)$ unter Regurgitationen und retrosternalen Schmerzen. Weitere 16,1\% $(n=5)$ klagten darüber, sich nur noch über weiche oder flüssige Kost ernähren zu können. 9,7\% (n=3) der Patienten zeigten Reflux, Globus pharyngis und Übelkeit auf. Unter Nachtschweiß, postprandialem Völlegefühl und Singultus litten 6,5\% (n=2). Aspiration, Räusperzwang, Heiserkeit, Schüttelfrost, Nachtrinken, postprandiales Bolusgefühl, postprandialer Würge- und Brechreiz oder Angst vor Nahrungsaufnahme bei gutem Appetit waren hingegen nur bei 3,2\% $(n=1)$ der Patienten zu finden (Abb. 17).

\subsection{Beschwerdedauer vor Behandlung}

Um ein strukturiertes Bild über die Dauer der prätherapeutischen Beschwerdesymptomatik zu bekommen, wurde das Patientenkollektiv $(n=48)$ in fünf Untergruppen mit 0-3 Monaten, 4-6 Monaten, 7-12 Monaten, 13-24 Monaten und >24 Monaten Beschwerdedauer unterteilt. Der Median der Beschwerdedauer lag bei allen 48 Patienten >24 Monaten, bei den Patienten der Gruppe UES $(n=17)$ bei 6,5 Monaten und bei den Patienten der Gruppe LES $(n=31)$ bei >24 Monaten (siehe Tab. 3). Der Median, auch als Zentralwert oder 50. Perzentil bekannt, ist der Wert der bei einer aufsteigenden Sortierung der Messwerte die Messreihe halbiert, so dass jeweils $50 \%$ der Messwerte ober- und unterhalb des Medians liegen (Pschyrembel 2007).

Tab. 3: Dauer der Beschwerdesymptomatik

\begin{tabular}{|c|c|c|c|c|c|c|c|}
\hline Dauer der Beschwerdesymptomatik & $\begin{array}{l}\text { UES } \\
n=17\end{array}$ & $\begin{array}{c}\text { neurogen } \\
n=7\end{array}$ & $\begin{array}{c}\text { Dystonie } \\
n=5\end{array}$ & $\begin{array}{c}\text { Tumor } \\
n=3\end{array}$ & $\begin{array}{c}\text { unklare } \\
\text { Genese } \\
n=2\end{array}$ & $\begin{array}{r}\text { LES } \\
\mathrm{n}=31\end{array}$ & 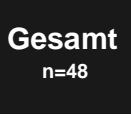 \\
\hline 0-3 Monate & $5(29 \%)$ & $5(71 \%)$ & 0 & 0 & 0 & $2(7 \%)$ & $7(15 \%)$ \\
\hline 4-6 Monate & $4(24 \%)$ & $1(14 \%)$ & 0 & $2(67 \%)$ & $1(50 \%)$ & $1(3 \%)$ & $5(10 \%)$ \\
\hline 7-12 Monate & 0 & 0 & 0 & 0 & 0 & $4(13 \%)$ & $4(8 \%)$ \\
\hline 13-24 Monate & $2(12 \%)$ & $1(14 \%)$ & 0 & $1(33 \%)$ & 0 & $2(7 \%)$ & $4(8 \%)$ \\
\hline$>24$ Monate & $6(35 \%)$ & 0 & $5(100 \%)$ & 0 & $1(50 \%)$ & $21(68 \%)$ & $27(56 \%)$ \\
\hline keine Angaben & 0 & 0 & 0 & 0 & 0 & $1(3 \%)$ & $1(2 \%)$ \\
\hline Median in Monaten & 6,5 & $0-3$ & $>24$ & $4-6$ & $>24$ & $>24$ & $>24$ \\
\hline
\end{tabular}




\subsubsection{Gruppe UES (oberer Ösophagussphinkter)}

Patienten der Gruppe UES ( $n=17)$ litten in 29,4\% $(n=5)$ 0-3 Monate, in 23,5\% $(n=4)$ 4-6 Monate, in 11,8\% $(n=2) 13-24$ Monate und in 35,3\% $(n=6)>24$ Monate an ihrer Symptomatik. Die Patienten der Untergruppe "neurogen" $(n=7)$ wiesen in $71,4 \%(n=5)$ der Fälle eine Beschwerdedauer von 0-3 Monaten und in 14,3\% $(n=1)$ jeweils 4-6 Monate und 13-24 Monate auf. Bei 100\% $(n=5)$ der Patienten in der Untergruppe "Dystonie" lag die Symptomatik schon länger als zwei Jahre, teilweise bis zu 20 Jahre, vor. Die Patienten der Untergruppe "Tumor" $(n=3)$ teilten sich zu 66,7\% ( $n=2)$ in 4-6 Monate und zu 33,3\% $(n=1)$ in 13-24 Monate auf. Zu jeweils $50 \%(n=1)$ verteilte sich die Patienten der Untergruppe „unklare Genese“ $(n=2)$ auf eine Beschwerdedauer von 4-6 Monaten und über 24 Monate auf (siehe Tab. 3).

\subsubsection{Gruppe LES (unterer Ösophagussphinkter)}

In der Gruppe LES ( $n=31)$ litt die Mehrheit der Patienten mit 67,7\% ( $n=21)$ länger als 24 Monate an einer Achalasie/“Nussknackerösophagus“. In 12,9\% (n=4) der Fälle bestand die Symptomatik 7-12 Monate und bei je 6,5\% ( $n=2)$ 0-3 Monate und 13-24 Monate. 3,2\% $(n=1)$ litten an der Symptomatik 4-6 Monate und bei 3,2\% $(\mathrm{n}=1)$ fanden sich keine Angaben.

Die Daten sind zusammengefasst in Tab. 3 dargestellt.

\subsection{Therapien vor Behandlung mit Botulinumtoxin $A$ in der Universitätsklinik Göttingen}

Die hier untersuchte Botulinumtoxintherapie war für einige Patienten nicht die erste Therapie; teilweise erhielten sie mehrere Therapien, so dass im Folgenden Mehrfachnennungen möglich sind. Zum einen handelt es sich bei den vorangegangenen Behandlungen um medikamentöse Therapien. Hierunter fallen Muskelrelaxanzien, wie z. B. Baclofen, Spasmolytika, wie z. B. Atropinsulfat, CalciumAntagonisten, wie z. B. Nifedipin, und Nitrate. Andere Therapieoptionen sind Logopädie, pneumatische Dilatation, Botulinumtoxin-A-Therapie, Myotomie, Trache- 
ostoma und PEG-Sonde. Die beiden zuletzt genannten sichern einerseits die Atemwege und andererseits die Ernährung. Die Daten sind in Tab. 4 zusammengefasst dargestellt.

Tab. 4: Vorangegangene Therapien bei Dysphagie

\begin{tabular}{|l|r|r|r|r|r|r|r|}
\hline Vorangegangene Therapien & $\begin{array}{c}\text { UES } \\
\mathbf{n = 1 7}\end{array}$ & $\begin{array}{c}\text { neurogen } \\
n=7\end{array}$ & $\begin{array}{c}\text { Dystonie } \\
n=5\end{array}$ & $\begin{array}{c}\text { Tumor } \\
n=3\end{array}$ & $\begin{array}{c}\text { unklare } \\
\text { Genese } \\
n=2\end{array}$ & $\begin{array}{c}\text { LES } \\
\mathbf{n = 3 1}\end{array}$ \\
\hline Gesamt \\
$\mathbf{n = 4 8}$
\end{tabular}

\subsubsection{Gruppe UES (oberer Ösophagussphinkter)}

In der Gruppe UES ( $n=17)$ bekamen 58,8\% $(n=10)$ der Patienten eine PEG-Sondeneinlage, und 41,2\% (n=7) trugen ein Tracheostoma. 47,1\% $(n=8)$ der Patienten waren in logopädischer Behandlung und 58,8\% $(n=10)$ wurden mit Medikamenten wie Trihexyphenidyl, Baclofen und Nifedipin behandelt. Eine vorherige Therapie mit Botulinumtoxin A hatten bereits 17,6\% $(n=3)$ der Patienten erfahren. Jeweils $5,9 \%(n=1)$ erhielten eine Glottiserweiterungs-Operation bzw. eine pneumatische Dilatation (siehe Tab. 4).

\subsubsection{Gruppe LES (unterer Ösophagussphinkter)}

Patienten der Gruppe LES ( $n=31)$ waren zu 38,7\% $(n=12)$ medikamentös mit Calciumantagonisten und Nitropräparaten eingestellt, 32,3\% $(n=10)$ versuchten es zuvor mit pneumatischer Dilatation und jeweils 3,2\% $(n=1)$ erfuhren zuvor eine Myotomie bzw. Botulinumtoxin-A-Injektion (siehe Tab. 4). 


\subsection{Injektionen und Dosierungen von Botulinumtoxin A}

Aufgrund der nachlassenden Wirkung von Botulinumtoxin A stellten sich einige Patienten mehr als einmal zur Injektionstherapie vor. Nicht nur die Anzahl der Vorstellungen variierte, auch die gewählte Dosierung musste teilweise angepasst werden, so dass die Dosierungen bei den unterschiedlichen Vorstellungen schwankten. So wurde v. a. im Bereich des UES in Absprache mit den Patienten die Dosierungen erhöht bzw. verringert, wenn die verspürte Wirkung bei der vorangegangenen Botulinumtoxin-A-Injektion noch nicht als ausreichend empfunden wurde bzw. unerwünschte Nebenwirkungen aufgetreten waren.

\subsubsection{Anzahl der Vorstellungen}

Anzahl der Vorstellungen

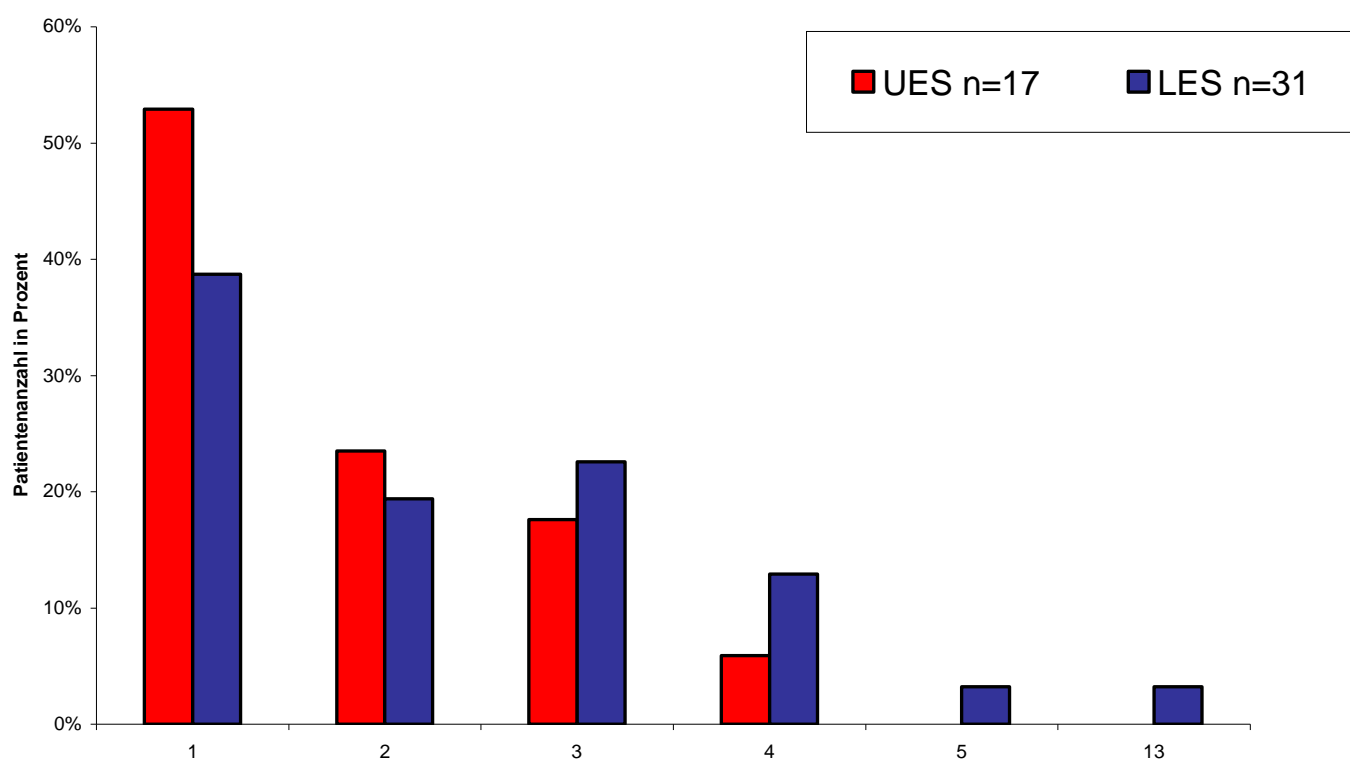

Abb. 18: Anzahl der Patientenvorstellungen in Prozent, Gruppe UES und LES im Vergleich

\subsubsection{Gruppe UES (oberer Ösophagussphinkter)}

In der Gruppe UES ( $n=17)$ haben sich die Patienten zu 52,9\% ( $n=9)$ einmalig, zu $23,5 \%(n=4)$ zweimalig, zu 17,6\% $(n=3)$ dreimalig und zu 5,9\% $(n=1)$ viermalig vorgestellt (siehe Abb. 18). 
Hauptgründe für eine einmalige Vorstellung $(n=9)$ waren zu 22,2\% $(n=2)$ mangelndes Ansprechen auf die Therapie bzw. zu 33,3\% ( $n=3)$ hielt die Wirkung bei Beobachtungsende noch an, so dass keine erneute Vorstellung notwendig war. Weitere Gründe waren zu jeweils 22,2\% ( $n=2)$ unbekannt verzogene Patienten und das Versterben der Patienten innerhalb von sechs Monaten nach der Therapie aufgrund seiner schwerwiegenden Grunderkrankung.

\subsubsection{Gruppe LES (unterer Ösophagussphinkter)}

In der Gruppe LES ( $n=31)$ haben sich die Patienten zu 38,7\% $(n=12)$ einmalig, zu $19,4 \%(n=6)$ zweimalig, zu 22,6\% ( $n=7)$ dreimalig und zu 12,9\% $(n=4)$ viermalig vorgestellt. Jeweils 3,2\% $(n=1)$ der Patienten kamen fünf Mal bzw. 13 Mal zur erneuten Botulinumtoxin-A-Injektion (siehe Abb. 18).

Die Hauptgründe für eine einmalige Vorstellung $(n=12)$ waren zu 33,3\% $(n=4)$ mangelndes Ansprechen auf die Therapie und zu 41,7\% $(n=5)$ hielt die Wirkung bei Beobachtungsende noch an, so dass keine erneute Vorstellung notwendig war. Weitere Gründe waren zu 16,7\% (n=2) unbekannt verzogene Patienten und zu 8,3\% $(n=1)$ ein Therapiewechsel.

\subsubsection{Dosierungen von Botulinumtoxin $\mathrm{A}$}

Tab. 5: Dosierung von Botulinumtoxin A bei Erstinjektion in Prozent Gruppe UES und LES im Vergleich

\begin{tabular}{|c|c|c|c|}
\hline Dosierung von Botulinumtoxin A & $\begin{array}{l}\text { UES } \\
n=17\end{array}$ & $\begin{array}{c}\text { LES } \\
n=31\end{array}$ & $\begin{array}{c}\text { Gesamt } \\
n=48\end{array}$ \\
\hline $30 \mathrm{U}$ & $5(29 \%)$ & 0 & $5(10 \%)$ \\
\hline $40 \mathrm{U}$ & $1(6 \%)$ & $1(3 \%)$ & $2(4 \%)$ \\
\hline $45 \mathrm{U}$ & $6(35 \%)$ & 0 & $6(13 \%)$ \\
\hline $50 \mathrm{U}$ & $2(12 \%)$ & 0 & $2(4 \%)$ \\
\hline $55 \mathrm{U}$ & $1(6 \%)$ & 0 & $1(2 \%)$ \\
\hline $65 \mathrm{U}$ & $1(6 \%)$ & 0 & $1(2 \%)$ \\
\hline $75 \mathrm{U}$ & $1(6 \%)$ & 0 & $1(2 \%)$ \\
\hline $100 \mathrm{U}$ & 0 & $25(81 \%)$ & $25(52 \%)$ \\
\hline keine Angaben & 0 & $5(16 \%)$ & $5(10 \%)$ \\
\hline
\end{tabular}




\subsubsection{Gruppe UES (oberer Ösophagussphinkter)}

Bei der ersten Injektion der Gruppe UES ( $n=17)$ wurden 30 U Botulinumtoxin A bei 29,4\% ( $n=5)$ der Patienten, $40 \cup$ in 5,9\% (n=1), $45 U$ in 35,3\% (n=6) und $50 U$ in $11,8 \%(n=2)$ der Fälle verabreicht. $55 \mathrm{U}, 65 \mathrm{U}$ und $75 \mathrm{U}$ Botulinumtoxin $A$ wurden zu jeweils 5,9\% ( $n=1)$ bei den Patienten injiziert, was zu einem Median von $45 \mathrm{U}$ führte (siehe Tab. 5). Bei der zweiten Injektion $(n=8)$ wurden $30 \cup$ Botulinumtoxin A zu 12,5\% ( $n=1), 45 \cup$ zu 50\% ( $n=4)$ und $60 \cup$ zu 37,5\% $(n=3)$ verabreicht, was einen Median von $45 \mathrm{U}$ ergab. Bei der dritten Injektion $(n=4)$ an Botulinumtoxin $A$ wurden $30 \mathrm{U}, 45 \mathrm{U}, 60 \mathrm{U}$ und $75 \mathrm{U}$ zu jeweils $25 \%(\mathrm{n}=1)$ injiziert. Bei der einzigen vierten Injektion wurden $45 \mathrm{U}$ zu 100\% ( $n=1)$ an Botulinumtoxin A appliziert.

\subsubsection{Gruppe LES (unterer Ösophagussphinkter)}

Die Dosierung bei der ersten Injektionstherapie bei den Patienten der Gruppe LES ( $n=31)$ mit $100 \cup$ Botulinumtoxin A wurde in 80,7\% ( $n=25)$ gewählt, $40 \cup$ wurden zu 3,2\% ( $n=1)$ injiziert und in 16,1\% $(n=5)$ der Fälle waren keine Angaben zu finden (siehe Tab. 5). Bei der zweiten Injektion ( $n=19)$ wurden $100 \mathrm{U}$ Botulinumtoxin in $68,4 \%(n=13)$ der Fälle injiziert. $80 \mathrm{U}$ an Botulinumtoxin wurden in 15,8\% $(n=3)$, $200 \mathrm{U}$ in $10,5 \%(n=2)$ und $20 \mathrm{U}$ in 5,3\% ( $n=1)$ der Fälle verabreicht. In der dritten Sitzung zur Botulinumtoxininjektion ( $n=13)$ wurden $100 \mathrm{U}$ in 84,6\% ( $n=11)$ und 200 $U$ in $7,7 \%(n=1)$ der Fälle appliziert. In weiteren $7,7 \%(n=1)$ der Fälle wurden keine Angaben zu der applizierten Menge an Botulinumtoxin gemacht. Bei der vierten Sitzung ( $n=6)$ wurden $100 \cup$ Botulinumtoxin $A$ in $83,3 \%(n=5)$ und $200 \cup$ in $16,7 \%$ $(n=1)$ der Fälle injiziert. In der fünften Sitzung $(n=2)$ wurden $100 \cup$ Botulinumtoxin A zu $100 \%(n=2)$ verabreicht.

\subsubsection{Zeitraum zwischen den Injektionen}

Der Zeitraum zwischen zwei Injektionen wurde, bei häufigeren Anwendungen, zur Systematisierung in verschiedene Zeitabschnitte geteilt. So ergaben sich sechs Gruppen von 0-60 Tagen, 61-90 Tagen, 91-120 Tagen, 121-150 Tagen, >150 Ta- 
gen und eine Gruppe, in der sich Patienten mit nur einmaliger Vorstellung befanden.

\subsubsection{Gruppe UES (oberer Ösophagussphinkter)}

Tab. 6: Gruppe UES, Dauer in Tagen bis zur erneuten Vorstellung zwischen der ersten und zweiten Injektion

\begin{tabular}{|l|r|}
\hline $\begin{array}{l}\text { Dauer bis zur erneuten } \\
\text { Vorstellung } \\
\text { Gruppe UES }\end{array}$ & $\begin{array}{r}\text { zwischen } \\
\mathbf{1}+\mathbf{+} . \\
\text { Injektion }\end{array}$ \\
\hline$<2$ Vorstellungen & $9(53 \%)$ \\
\hline 0-60 Tage & $1(6 \%)$ \\
\hline 61-90 Tage & $2(12 \%)$ \\
\hline 91-120 Tage & $1(6 \%)$ \\
\hline $121-150$ Tage & $1(6 \%)$ \\
\hline$>150$ Tage & $3(18 \%)$ \\
\hline
\end{tabular}

Tab. 7: Gruppe UES, Dauer in Tagen bis zur erneuten Vorstellung zwischen der zweiten und dritten Injektion

\begin{tabular}{|l|r|}
\hline $\begin{array}{l}\text { Dauer bis zur erneuten } \\
\text { Vorstellung } \\
\text { Gruppe UES }\end{array}$ & $\begin{array}{c}\text { zwischen } \\
\mathbf{2}+\mathbf{+} . \\
\text { Injektion }\end{array}$ \\
\hline$<3$ Vorstellungen & $13(77 \%)$ \\
\hline 0-60 Tage & 0 \\
\hline 61-90 Tage & 0 \\
\hline 91-120 Tage & $2(12 \%)$ \\
\hline 121-150 Tage & 0 \\
\hline$>150$ Tage & $2(12 \%)$ \\
\hline
\end{tabular}

Im Kollektiv der Gruppe UES ( $\mathrm{n}=17)$ stellten sich zwischen der ersten und der zweiten Injektion 5,9\% $(\mathrm{n}=1)$ der Patienten bereits nach 0-60 Tagen und 11,8\% $(\mathrm{n}=2)$ nach 61-90 Tagen vor. Zu jeweils 5,9\% $(\mathrm{n}=1)$ wurden die Patienten nach 91120 Tagen und nach 121-150 Tagen erneut vorstellig, 17,6\% $(n=3)$ kamen nach $>150$ Tagen wieder. Die verbliebenen 52,9\% $(n=9)$ der Patienten stellten sich nur einmalig vor (siehe Tab. 6).

Zwischen der zweiten und dritten Injektion stellten sich jeweils 11,8\% $(n=2)$ nach 91-120 Tagen und nach über 150 Tagen und wieder vor. Die verbliebenen 76,5\% $(n=13)$ der Patienten stellten sich nur ein- bis zweimalig in der Sprechstunde vor (siehe Tab. 7). 


\subsubsection{Gruppe LES (unterer Ösophagussphinkter)}

Tab. 8: Gruppe LES, Dauer in Tagen bis zur erneuten Vorstellung zwischen der ersten und zweiten Injektion

\begin{tabular}{|l|r|}
\hline $\begin{array}{l}\text { Dauer bis zur erneuten } \\
\text { Vorstellung } \\
\text { Gruppe LES }\end{array}$ & $\begin{array}{c}\text { zwischen } \\
\mathbf{1 .}+\mathbf{2} . \\
\text { Injektion }\end{array}$ \\
\hline$<2$ Vorstellungen & $12(39 \%)$ \\
\hline 0-60 Tage & $3(10 \%)$ \\
\hline 61-90 Tage & $3(10 \%)$ \\
\hline 91-120 Tage & 0 \\
\hline 121-150 Tage & $4(13 \%)$ \\
\hline$>150$ Tage & $9(29 \%)$ \\
\hline
\end{tabular}

Tab. 9: Gruppe LES, Dauer in Tagen bis zur erneuten Vorstellung zwischen der zweiten und dritten Injektion

\begin{tabular}{|l|r|}
\hline $\begin{array}{l}\text { Dauer bis zur erneuten } \\
\text { Vorstellung } \\
\text { Gruppe LES }\end{array}$ & $\begin{array}{c}\text { zwischen } \\
\mathbf{2}+\mathbf{+} \text {. } \\
\text { Injektion }\end{array}$ \\
\hline$<3$ Vorstellungen & $18(58 \%)$ \\
\hline 0-60 Tage & $3(10 \%)$ \\
\hline 61-90 Tage & 0 \\
\hline 91-120 Tage & 0 \\
\hline 121-150 Tage & $2(7 \%)$ \\
\hline$>150$ Tage & $8(26 \%)$ \\
\hline
\end{tabular}

Tab. 10: Gruppe LES, Dauer in Tagen bis zur erneuten Vorstellung zwischen der dritten und vierten Injektion

\begin{tabular}{|l|r|}
\hline $\begin{array}{l}\text { Dauer bis zur erneuten } \\
\text { Vorstellung } \\
\text { Gruppe LES }\end{array}$ & $\begin{array}{c}\text { zwischen } \\
\mathbf{3}+\mathbf{4} . \\
\text { Injektion }\end{array}$ \\
\hline$<4$ Vorstellungen & $25(81 \%)$ \\
\hline $0-60$ Tage & 0 \\
\hline 61-90 Tage & $1(3 \%)$ \\
\hline 91-120 Tage & $1(3 \%)$ \\
\hline 121-150 Tage & 0 \\
\hline$>150$ Tage & $4(13 \%)$ \\
\hline
\end{tabular}

Im Kollektiv der Gruppe LES ( $n=31)$ stellten sich jeweils 9,7\% $(n=3)$ der Patienten nach 0-60 Tagen und nach 61-90 Tagen erneut vor. 12,9\% $(n=4)$ wurde nach 121150 Tagen wieder vorstellig. Weitere 29,0\% ( $n=9)$ wurden im Zeitraum nach >150 Tagen erneut vorstellig und die verbliebenen 38,7\% $(n=12)$ der Patienten stellten sich nur einmalig vor (siehe Tab. 8).

Der Zeitabstand von der zweiten zur dritten Injektion war in 9,7\% $(n=3)$ der Fälle 060 Tagen und in 6,5\% $(n=2)$ ein Zeitraum zwischen 121 und 150 Tagen. 25,8\% $(n=8)$ der Patienten wurden erst nach >150 Tagen wieder vorstellig und 58,1\% $(n=18)$ der Patienten stellten sich kein drittes Mal erneut vor (siehe Tab. 9). Wäh- 
rend des Zeitraumes von der dritten zur vierten Injektion erhielten 80,7\% $(n=25)$ der Patienten maximal drei Injektionen, 12,9\% $(n=4)$ zeigten einen Zeitraum von über 150 Tagen und jeweils 3,2\% $(n=1)$ einen Zeitraum von 61-90 Tagen sowie 91-120 Tagen bis zur erneuten Wiedervorstellung (siehe Tab. 10).

\subsubsection{Dosismodifikation und Intervallveränderungen im Verlauf bei häufigeren Anwendungen}

Neben der Anzahl der Vorstellungen, der Dosierungen und dem Zeitraum zwischen den Injektionen wurden im Folgenden mögliche Dosis- und Intervallveränderungen untersucht. Es wurde in der Dosierung zwischen Eskalation, Reduktion und keine Veränderung, sowie bei dem Intervallvergleich zwischen Verlängerung, Verkürzung und keine Veränderung unterschieden. Die Ergebnisse sind in den Tab. 11 und 12 zu finden.

Tab. 11: Dosismodifikation zwischen den Injektionen

\begin{tabular}{|l|r|r|r|}
\hline Dosismodiflkation & $\begin{array}{r}\text { UES } \\
\mathbf{n = 1 7}\end{array}$ & \multicolumn{1}{c|}{ LES } \\
$\mathbf{n = 3 1}$ & $\begin{array}{c}\text { Gesamt } \\
\mathbf{n = 4 8}\end{array}$ \\
\hline Einmalige Injektion & $9(53 \%)$ & $12(39 \%)$ & $\mathbf{2 1}(\mathbf{4 4 \% )}$ \\
\hline keine Änderung & $4(24 \%)$ & $16(52 \%)$ & $\mathbf{2 0}(\mathbf{4 2 \% )}$ \\
\hline Eskalation & $4(24 \%)$ & $3(10 \%)$ & $\mathbf{7}(\mathbf{1 5 \% )}$ \\
\hline Reduktion & 0 & 0 & $\mathbf{0}$ \\
\hline
\end{tabular}

Tab. 12: Intervallmodifikation zwischen den Injektionen

\begin{tabular}{|l|r|r|r|}
\hline Intervallmodifikation & $\begin{array}{r}\text { UES } \\
\mathbf{n = 1 7}\end{array}$ & \multicolumn{1}{c|}{$\begin{array}{c}\text { LES } \\
\mathbf{n = 3 1}\end{array}$} & $\begin{array}{c}\text { Gesamt } \\
\mathbf{n = 4 8}\end{array}$ \\
\hline Unter 3 Injektionen & $12(71 \%)$ & $18(58 \%)$ & $\mathbf{3 0}(\mathbf{6 3} \%)$ \\
\hline keine Änderung & $2(12 \%)$ & $4(13 \%)$ & $\mathbf{6}(\mathbf{1 3} \%)$ \\
\hline Verkürzung & $2(12 \%)$ & $6(19 \%)$ & $\mathbf{8}(\mathbf{1 7 \%})$ \\
\hline Verlängerung & $1(6 \%)$ & $3(10 \%)$ & $\mathbf{4}(\mathbf{8 \%})$ \\
\hline
\end{tabular}

\subsubsection{Gruppe UES (oberer Ösophagussphinkter)}

In der Gruppe UES stellten sich 52,9\% (n=9) der Patienten nur einmalig vor und bei jeweils 23,5\% ( $n=4)$ kam es zu keiner Veränderung bzw. zu einer Erhöhung der Dosierung. Eine Reduzierung wurde hingegen nicht vorgenommen. 
Im Vergleich der Intervalle stellten sich 70,6\% $(n=12)$ lediglich ein bis zwei Mal im Universitätsklinikum Göttingen vor. Bei jeweils 11,8\% (n=2) konnte keine Veränderung des Intervalls bzw. eine Verkürzung des Zeitraumes festgestellt werden. Bei den verbliebenen 5,9\% ( $n=1)$ zeigte sich eine Intervallverlängerung.

\subsubsection{Gruppe LES (unterer Ösophagussphinkter)}

In der Gruppe LES haben sich 38,7\% ( $n=12)$ nur einmalig vorgestellt. Es zeigt sich bei $51,6 \%(n=16)$ keine Veränderung und bei $9,7 \%(n=3)$ eine Erhöhung der Dosierung.

Bei Betrachtung des Zeitintervalls stellten sich $58,1 \%(n=18)$ lediglich ein bis zwei Mal vor. Bei 12,9\% $(n=4)$ wurde keine Änderung des Zeitintervalls vorgenommen, $19,4 \%(n=6)$ der Patienten verkürzten den Zeitraum bis zur erneuten Vorstellung und $9,7 \%(n=3)$ verlängerten den Zeitraum zwischen den Injektionen.

\subsection{Dauer bis zum Wirkungseintritt}

Die Angaben zur Wirkung wurden aus den archivierten Akten entnommen bzw. im Telefonat subjektiv erfragt. Hierbei wurden entweder die Patienten selbst in $85,4 \%$ $(\mathrm{n}=41)$ der Fälle bzw. deren Ehepartner, Eltern, Kinder oder Pflegepersonal in $6,3 \%(n=3)$ der Fälle befragt. 8,3\% $(n=4)$ konnten aufgrund eines Umzugs an einen unbekannten Ort nicht mehr befragt werden. Aufgrund teilweise langer Zeitspannen zwischen Therapie und Erhebung der Daten wurde keine nähere Differenzierung der Wirkung erhoben. Es wurde lediglich erhoben, ob die Patienten eine Verbesserung der Dysphagie durch die Therapie verspürten oder nicht. Das beobachtete Spektrum der Ergebnisse reichte dabei von nur dezenten Verbesserungen, so dass weiterhin keine feste Nahrung geschluckt werden konnte, bis hin zur vollkommenen Beschwerdefreiheit.

Eine weitere Differenzierung wurde zum Wirkungseintritt gemacht. Hier konnten die Patienten in sieben Gruppen eingeteilt werden, abhängig von der Zeitspanne des Wirkungseintritts. So konnte der Zeitrahmen in 0-2 Tage, 3-5 Tage, 6-8 Tage 
und >8 Tage unterteilt werden. Darüber hinaus kamen noch die ergänzenden Gruppen „bei verspürter Wirkung keine Angabe zum Zeitpunkt des Wirkungseintritts”, „kein Wirkungseintritt” und „keine Angabe zur Wirkung“ hinzu. Letztere Untergruppierung $(n=3)$ bestand aus Patienten, die unbekannt verzogen waren und daher nicht mehr weiter verfolgt werden konnten. Sie wurde aus der weiteren Berechnung, zur Vermeidung von Verzerrungseffekten im Endergebnis, entfernt (siehe Tab. 13).

Tab. 13: Dauer des Wirkungseintritts nach Botulinumtoxin-A-Injektion

\begin{tabular}{|l|r|r|r|}
\hline Wirkungseintritt & $\begin{array}{c}\text { UES } \\
\mathbf{n}=\mathbf{1 5}\end{array}$ & $\begin{array}{c}\text { LES } \\
\mathbf{n}=\mathbf{3 0}\end{array}$ & $\begin{array}{c}\text { Gesamt } \\
\mathbf{n}=\mathbf{4 5}\end{array}$ \\
\hline 0-2 Tage & $4(27 \%)$ & $17(57 \%)$ & $\mathbf{2 1}(\mathbf{4 7} \%)$ \\
\hline 3-5 Tage & $1(7 \%)$ & $4(13 \%)$ & $\mathbf{5}(\mathbf{1 1} \%)$ \\
\hline 6-8 Tage & $2(13 \%)$ & 0 & $\mathbf{2}(\mathbf{4} \%)$ \\
\hline > 8 Tage & $2(13 \%)$ & 0 & $\mathbf{2}(\mathbf{4} \%)$ \\
\hline keine Angaben zum Zeitpunkt des Wirkungseintritts & $2(13 \%)$ & $2(7 \%)$ & $\mathbf{4}(\mathbf{9} \%)$ \\
\hline kein Wirkungseintritt & $4(27 \%)$ & $7(23 \%)$ & $\mathbf{1 1 ( 2 4 \% )}$ \\
\hline
\end{tabular}

\subsubsection{Gruppe UES (oberer Ösophagussphinkter)}

Bei Patienten der Gruppe UES ( $n=17)$ waren $11,8 \%(n=2)$ aus der Untergruppierung „keine Angabe zur Wirkung“. Nach Entfernung dieser zwei Patienten besteht die Gruppe UES noch aus $n=15$ Patienten. Bei insgesamt 73,3\% $(n=11)$ der Patienten zeigte sich eine Wirkung auf Botulinumtoxin A.

Bei 26,7\% $(n=4)$ der Patienten stellte sich die Wirkung bereits nach 0-2 Tagen ein und bei 6,7\% $(n=1)$ zeigte sich nach 3-5 Tagen eine Wirkung. Jeweils 13,3\% $(n=2)$ der Patienten berichteten von einem Wirkungseintritt innerhalb von 6-8 Tagen bzw. nach über 8 Tagen. Weitere $13,3 \%(n=2)$ konnten trotz verspürter Wirkung keine Angabe zum Zeitpunkt des Wirkungseintritts machen und bei 26,7\% $(n=4)$ wurde kein Wirkungseintritt beobachtet (siehe Tab. 13).

Zur näheren Darstellung eines Therapieerfolges wird auf nachfolgende Abb. 19 hingewiesen. 


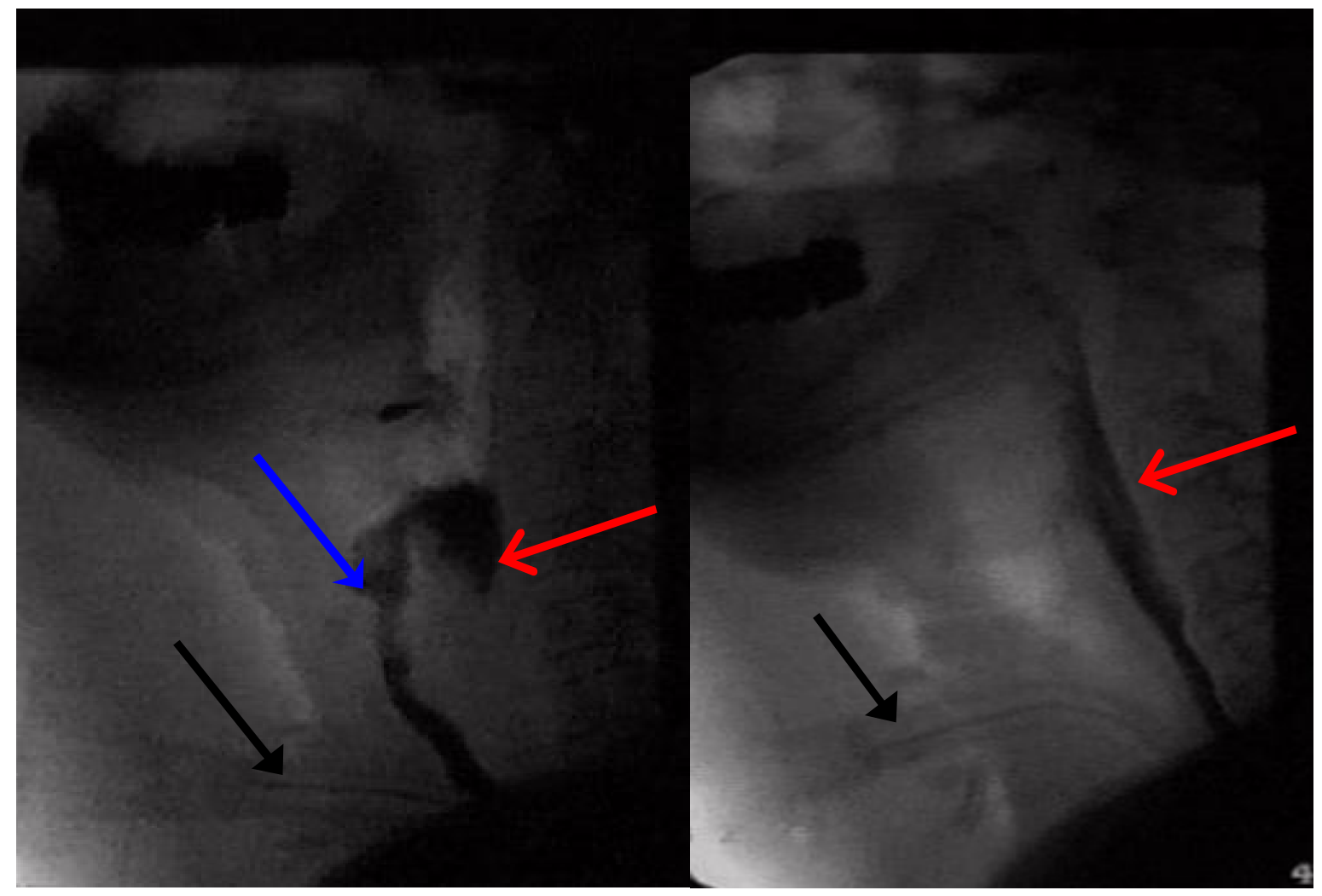

Abb. 19: Videofluoroskopie des UES vor und nach der Behandlung mit Botulinumtoxin

Linkes Bild (vor Behandlung mit Botulinumtoxin A): Man erkennt den Eingangsbereich des Ösophagus mit funktioneller Stenosierung $\rightarrow$. Hier kann das flüssige Kontrastmittel nicht den UES passieren, es kommt zum Aufstau im Hypopharynx mit Übertritt in die Trachea $\rightarrow$ was zur maximalen Aspiration führen würde, wäre die erkennbare Trachealkanüle nicht geblockt $\rightarrow$.

Rechtes Bild (nach Behandlung mit Botulinumtoxin A): Man erkennt einen gut passierbaren UES $\rightarrow$ ohne Einengung, Aufstau und Übertritt des Kontrastmittels in die Trachea.

\subsubsection{Gruppe LES (unterer Ösophagussphinkter)}

Bei Patienten der Gruppe LES wurde in 3,2\% $(n=1)$ keine Angabe zur Wirkung gemacht. So wurde dieser Patient zur weiteren Berechnung aus der Gruppe LES entfernt $(n=30)$. Es zeigte sich bei 76,7\% $(n=23)$ der Patienten eine Wirkung auf Botulinumtoxin A.

Bei 56,7\% ( $n=17)$ der Patienten stellte sich die Wirkung bereits nach 0-2 Tagen ein, 13,3\% $(n=4)$ berichteten von einem Wirkungseintritt nach 3-5 Tagen und bei $6,7 \%(n=2)$ wurden keine Angaben zum Zeitpunkt des Wirkungseintritts ange- 
geben. Weitere $23,3 \%(n=7)$ der Patienten konnten keinen Wirkungseintritt angeben. Einen Überblick über den Wirkungseintritt soll Tab. 13 geben.

Es fällt auf, dass Patienten der Gruppe LES einen wesentlich früheren Wirkungseintritt verspürten als Patienten der Gruppe UES, die teilweise über eine Woche brauchten, um die volle Wirkungsentfaltung zu spüren. Zur genaueren Betrachtung sind die Dosierungen innerhalb der Gruppe UES $(n=15)$ analysiert worden. Bei den Patienten, die innerhalb der ersten fünf Tage $(n=5)$ nach Botulinumtoxin-A-Injektion von einer vollen Wirkung sprachen, wurden durchschnittlich $40 \mathrm{U}$ bzw. ein Median von $45 \mathrm{U}$ Botulinumtoxin A injiziert. Bei diesen Patienten wurden an vorherigen Behandlungen bisher neben manueller Therapie $(n=1)$ auch medikamentöse Therapie versucht $(n=3)$. Bei Patienten, die erst nach sechs Tagen und länger $(n=4)$ nach der Injektion von Botulinumtoxin A die volle Wirkung angaben, wurden hingegen durchschnittlich 53,75 U bzw. ein Median von $55 \cup$ Botulinumtoxin $A$ injiziert. Diese Patienten hatten an vorhergehenden Therapie PEG-Sonden ( $n=3)$, ein Tracheostoma $(n=1)$ oder eine Provoxstimmprothese $(n=1)$ erhalten. Bei einem Patienten wurde eine Glottiserweiterungs-Operation versucht. Zusätzlich erhielten sie Logopädie $(n=2)$ und medikamentöse Therapie $(n=3)$.

\subsection{Vergleich des Therapieerfolgs von subjektiven Aussagen und objektiver Diagnostik}

Vom gesamten Patientenkollektiv $(n=48)$ erhielten $36,6 \%(n=19)$ der Patienten nach der Injektionstherapie erneute Diagnostik um den Therapieerfolg zu objektivieren. Diese erfolgte bei unterschiedlichen Patienten sowohl nach der 1., 2., 3. als auch nach der 5. Injektion, so dass Mehrfachnennungen unter den Patienten, die nachfolgende Diagnostik erfuhren, möglich sind. Im gesamten Patientenkollektiv wurden die Nachuntersuchungen in einem unterschiedlichen Zeitrahmen postinterventionell durchgeführt. Sie reichten von einer Bariumbrei-Kontrastuntersuchung direkt nach der Botulinumtoxin-A-Injektion $(n=1)$ bis hin zur nachfolgenden Ösophagogastroduodenoskopie elf Monate später $(n=1)$. Der Median für den beobachteten Zeitraum nach der Botulinumtoxin-A-Injektion lag bei sechs Wochen. Über diese Beobachtungen konnte in $n=25$ Fällen ein bedingter 
Vergleich von subjektiver Aussage mit objektiver Diagnostik gemacht werden, wobei eine Übereinstimmung bzw. keine Übereinstimmung in subjektiver und objektiver „Diagnostik“ bei Erfolg und Misserfolg ausgewertet wurde. Insgesamt konnten fünfzehn Patienten aus beiden Gruppen nach der ersten Injektion $(n=15)$, sechs Patienten nach der zweiten Injektion $(n=6)$ und jeweils zwei Patienten nach der dritten $(n=2)$ und fünften Injektion $(n=2)$ verglichen werden. Insgesamt zeigten $84,0 \%(n=21)$ eine Übereinstimmung von objektiver Diagnostik und subjektiver Aussage. Keine Übereinstimmung zeigten insgesamt 16,0\% ( $n=4)$. Zum genauen Vergleich wird auf die folgende Tab. 14 verwiesen.

Tab. 14: Übereinstimmung von objektivem und subjektivem Therapieergebnis

\begin{tabular}{|l|r|r|r|r|}
\hline $\begin{array}{l}\text { Übereinstimmung von objektivem und } \\
\text { subjektivem Therapieergebnis }\end{array}$ & $\begin{array}{c}\text { 1.Injektion } \\
\mathbf{n = 1 5}\end{array}$ & $\begin{array}{c}\text { 2.Injektion } \\
\mathbf{n = 6}\end{array}$ & $\begin{array}{c}\text { 3.Injektion } \\
\mathbf{n = 2}\end{array}$ & $\begin{array}{c}\text { 5.Injektion } \\
\mathbf{n = 2}\end{array}$ \\
\hline keine Übereinstimmung & $\mathbf{2}$ & $\mathbf{2}$ & $\mathbf{0}$ & $\mathbf{0}$ \\
\hline Diagnostikum: Videofluoroskopie (UES) & 1 & 0 & 0 & 0 \\
\hline Diagnostikum: Ösophagogastroduodenoskopie (LES) & 0 & 2 & 0 & 0 \\
\hline Diagnostikum: Computertomographie (LES) & 1 & 0 & 0 & 0 \\
\hline Diagnostikum: Manometrie (LES) & 0 & 0 & 0 & 0 \\
\hline Übereinstimmung & $\mathbf{1 3}$ & $\mathbf{4}$ & $\mathbf{2}$ & $\mathbf{2}$ \\
\hline Diagnostikum: Videofluoroskopie (UES) & 3 & 0 & 0 & 0 \\
\hline Diagnostikum: Ösophagogastroduodenoskopie (LES) & 6 & 2 & 0 & 1 \\
\hline Diagnostikum: Bariumbrei-Kontrastuntersuchung (LES) & 1 & 0 & 1 & 0 \\
\hline Diagnostikum: Manometrie (LES) & 3 & 2 & 1 & 1 \\
\hline
\end{tabular}

\subsubsection{Gruppe UES (oberer Ösophagussphinkter)}

In der Gruppe UES ( $n=17)$ wurde diese bei 23,5\% $(n=4)$ der Patienten mittels der Videofluoroskopie durchgeführt. Von diesen vier Patienten $(n=4)$ zeigten $75,0 \%$ $(n=3)$ eine Übereinstimmung und 25,0\% $(n=1)$ keine Übereinstimmung von subjektiver Aussage und objektiver Diagnostik.

\subsubsection{Gruppe LES (unterer Ösophagussphinkter)}

In der Gruppe LES ( $n=31)$ wurde bei $67,7 \%(n=21)$ eine postinterventionelle Diagnostik durchgeführt. Die Diagnostik wurde bei den 21 beobachteten Fällen in $52,4 \%(n=11)$ über Ösophagogastroduodenoskopie, in 33,3\% ( $n=7)$ über Manometrie, in $9,5 \%(n=2)$ über Bariumbrei-Kontrastuntersuchung und in 4,8\% $(n=1)$ 
über CT durchgeführt. Dabei zeigten $85,7 \%(n=18)$ eine Übereinstimmung und $14,3 \% \quad(n=3)$ keine Übereinstimmung von subjektiver Aussage und objektiver Diagnostik.

\subsection{Unerwünschte Wirkungen von Botulinumtoxin A}

Unerwünschte Wirkungen zeigten sich innerhalb des gesamten Patientenkollektivs $(n=48)$ in $8,3 \%(n=4)$ der Fälle.

\subsubsection{Gruppe UES (oberer Ösophagussphinkter)}

Patienten der Gruppe UES ( $n=17)$ stellten sich zu 5,9\% $(n=1)$ mit unerwünschten Wirkungen, wie z. B. einer hängenden Lippe und Singultus vor. Dieser Patient wurde zusätzlich zur intramuskulären Injektion in den M. cricopharyngeus auch im Bereich beider Musculi masseter, im Mundboden, im Bereich caudal-perioral und im Bereich des Platysmas mit Botulinumtoxin A behandelt.

\subsubsection{Gruppe LES (unterer Ösophagussphinkter)}

Patienten der Gruppe LES ( $n=31)$ stellten sich in 9,7\% $(n=3)$ der Fälle nach der ersten Injektion mit Sodbrennen und Reflux vor. Nach der zweiten Injektion wurden darüber hinaus bei 3,2\% der Patienten $(n=1)$ eine langstreckige Ösophagitis und streifige Antrumgastritis in der Ösophagogastroduodenoskopie festgestellt. Zusätzlich klagten $3,2 \%$ der Patienten $(n=1)$ über ein Fortbestehen der retrosternalen Schmerzen bei einer Wirkung vergleichbar mit der pneumatischen Dilatation.

\subsection{Vergleich des Patientenkollektivs anhand von Respon- dern und Non-Respondern}

In der folgenden Tab. 15 sind Responder und Non-Responder gegenübergestellt. In die Gruppe der Responder sind alle Patienten aufgenommen, die nach Botuli- 
numtoxin-A-Injektionen eine Verbesserung ihrer Schluckstörung verspürten. In die Gruppe der Non-Responder sind dagegen die Patienten aufgenommen, die keinen Effekt bzw. einen negativen Effekt innerhalb des beobachteten Zeitraumes auf Botulinumtoxin A verspürten.

Insgesamt konnten 45 der 48 Patienten miteinander verglichen werden. Drei Patienten, zwei Patienten aus der Gruppe UES, wobei eine Patientin der Subgruppe „Dystonie“ und ein Patient der Subgruppe „neurogen“ angehörte, sowie ein Patient aus der Gruppe LES, sind im Verlauf unbekannt verzogen und daher von der weiteren Beobachtung ausgenommen worden.

In dieser Gegenüberstellung fiel u. a. auf, dass das Gesamtkollektiv bei den Respondern um 4,6 Jahre jünger war. Bei der Beschwerdedauer zeigten sich wenige Unterschiede im Gesamtkollektiv. Es ergab sich kein wesentlicher Unterschied bei der Patientenanzahl mit Aspirationen und Gewichtsverlust. Hingegen zeigten die Responder eine höhere Anzahl an vorhergehender Therapien: 44,1\% $(n=15)$ versuchten bei den Respondern $(n=34)$ eine Therapie vor Botulinumtoxin $A$ und 9,1\% $(n=1)$ bei den Non-Respondern $(n=11)$. Ein wesentlicher Unterschied in der applizierten Dosierung an Botulinumtoxin A ließ sich nicht feststellen (siehe Tab. 15 und Tab. 16).

Tab. 15: Übersicht über das Patientenkollektiv der Responder

\begin{tabular}{|c|c|c|c|c|c|c|c|}
\hline RESPONDER & UES & neurogen & Dystonie & Tumor & $\begin{array}{l}\text { unklare } \\
\text { Genese }\end{array}$ & LES & Gesamt \\
\hline Anzahl Patienten & 11 & 5 & 2 & 2 & 2 & 23 & 34 \\
\hline Durchschnittsalter in Jahren & 59,4 & 66,6 & 50 & 53 & 57 & 57,6 & 58,2 \\
\hline Verhältnis Mann:Frau & $10: 1$ & $5: 0$ & $1: 1$ & $2: 0$ & $2: 0$ & $15: 8$ & $25: 9$ \\
\hline durchschnittliche Beschwerdedauer & $2,6 \mathrm{~J}^{*}$ & $3 \mathrm{Mo}^{*}$ & $10 J^{*}$ & $12 \mathrm{Mo}^{*}$ & $3 J^{*}$ & $9,1 \mathrm{~J}^{*}$ & $7,0 \mathrm{~J}^{*}$ \\
\hline Patientenanzahl mit Gewichtsverlust & 2 & 0 & 1 & 1 & 0 & 14 & 16 \\
\hline Patientenanzahl mit Aspiration & 7 & 3 & 1 & 2 & 1 & 0 & 7 \\
\hline $\begin{array}{l}\text { Patientenanzahl vorheriger Botox®- } \\
\text { Behandlungen }\end{array}$ & 2 & 0 & 2 & 0 & 0 & 1 & 3 \\
\hline Patientenanzahl vorheriger Dilatationen & 1 & 1 & 0 & 0 & 0 & 10 & 11 \\
\hline Patientenanzahl vorheriger Myotomien & 0 & 0 & 0 & 0 & 0 & 1 & 1 \\
\hline $\begin{array}{l}\text { Patientenanzahl mit } \\
\text { unerwünschten Wirkungen }\end{array}$ & 1 & 0 & 1 & 0 & 0 & 3 & 4 \\
\hline durchschnittliche Menge an Botox® & $45,5 \cup$ & $51 U$ & $37,5 \mathrm{U}$ & $47,5 U$ & $37,5 \cup$ & $99,1 \cup$ & $81,7 \mathrm{U}$ \\
\hline
\end{tabular}


Tab. 16: Übersicht über das Patientenkollektiv der Non-Responder

\begin{tabular}{|c|c|c|c|c|c|c|c|}
\hline NON-RESPONDER & UES & neurogen & Dystonie & Tumor & $\begin{array}{l}\text { unklare } \\
\text { Genese }\end{array}$ & LES & Gesamt \\
\hline Anzahl Patienten & 4 & 1 & 2 & 1 & 0 & 7 & 11 \\
\hline Durchschnittsalter in Jahren & 69,25 & 69 & 67 & 74 & 0 & 59,1 & 62,8 \\
\hline Verhältnis Mann:Frau & 2:2 & 1:0 & $0: 2$ & 1:0 & 0 & $5: 2$ & $7: 4$ \\
\hline durchschnittliche Beschwerdedauer & $6,9 \mathrm{~J}^{*}$ & $1 \mathrm{Mo}^{*}$ & $13,5 \mathrm{~J}^{*}$ & $6 \mathrm{Mo}^{*}$ & 0 & $6,4 \mathrm{~J}^{*}$ & $6,6 \mathrm{~J}^{*}$ \\
\hline Patientenanzahl mit Gewichtsverlust & 3 & 1 & 1 & 1 & 0 & 2 & 5 \\
\hline Patientenanzahl mit Aspiration & 3 & 1 & 2 & 0 & 0 & 1 & 4 \\
\hline $\begin{array}{l}\text { Patientenanzahl vorheriger Botox@- } \\
\text { Behandlungen }\end{array}$ & 1 & 0 & 0 & 1 & 0 & 0 & 1 \\
\hline Patientenanzahl vorheriger Dilatationen & 0 & 0 & 0 & 0 & 0 & 0 & 0 \\
\hline Patientenanzahl vorheriger Myotomien & 0 & 0 & 0 & 0 & 0 & 0 & 0 \\
\hline $\begin{array}{l}\text { Patientenanzahl mit } \\
\text { unerwünschten Wirkungen }\end{array}$ & 1 & 0 & 1 & 0 & 0 & 0 & 1 \\
\hline durchschnittliche Menge an Botox® & $40 \mathrm{U}$ & $55 \mathrm{U}$ & $30 \mathrm{U}$ & $45 \mathrm{U}$ & 0 & $100 \mathrm{U}$ & $78,2 \mathrm{U}$ \\
\hline
\end{tabular}

\subsubsection{Gruppe UES (oberer Ösophagussphinkter)}

Im Vergleich der Gruppe UES ( $n=15)$ zwischen Respondern 73,3\% $(n=11)$ und Non-Respondern 26,7\% $(n=4)$ zeigte sich ein um 9,85 Jahre jüngeres Kollektiv bei den Respondern. Die Beschwerdedauer war dagegen bei den Non-Respondern im Durchschnitt um 4,3 Jahre länger. Weiterhin ließ sich bei den Non-Respondern mit 75,0\% ( $n=3)$ eine höhere Rate an Patienten mit Gewichtsverlust im Verhältnis zu den Respondern mit 18,2\% ( $n=2)$ feststellen. Hingegen zeigte sich die Anzahl der Patienten mit Aspirationen sowohl bei den Respondern mit 63,6\% ( $n=7)$ als auch bei den Non-Respondern mit $75 \%(n=3)$ relativ ausgeglichen. Keinen Unterschied fand sich bei der Anzahl der vorhergehenden Therapien. Hingegen war die Dosierung bei den Non-Respondern mit durchschnittlich $40 \mathrm{U}$ etwas niedriger im Vergleich zu den Respondern mit durchschnittlich 45,5 U (siehe Tab. 15 und Tab. 16).

\subsubsection{Gruppe LES (unterer Ösophagussphinkter)}

Im Vergleich der Gruppe LES ( $n=30)$ zwischen Respondern 76,7\% $(n=23)$ und Non-Respondern $23,3 \%(n=7)$ zeigte sich eine relativ ausgeglichene Altersverteilung mit einem um 1,5 Jahre jüngeren Kollektiv bei den Respondern. Die Beschwerdedauer war bei den Respondern im Durchschnitt um 2,7 Jahre länger. 
Ebenso fand sich bei den Respondern mit 60,9\% ( $n=14)$ eine höhere Rate an Patienten mit Gewichtsverlust als bei den Non-Respondern mit 28,6\% ( $n=2)$. Hingegen fanden sich 14,3\% $(n=1)$ bei den Non-Respondern mit Aspiration im Vergleich zu den Respondern mit 0,0\%. Bei der Anzahl der vorhergehenden Therapien hatten bereits $52,2 \%(n=12)$ der Responder einen Therapieversuch hinter sich im Vergleich zu den Non-Respondern bei denen 0,0\% eine vorhergehende Therapie versuchten. Kein Unterschied zeigte sich bei der Dosierung (siehe Tab. 15 und Tab. 16). 


\section{$4 \quad$ DISKUSSION}

\subsection{Wirkung von Botulinumtoxin A auf die Dysphagie}

Ziel der Behandlung mit Botulinumtoxin A war eine Reduktion der Dysfunktion der Ösophagussphinkteren unterschiedlicher Genese. Über die Induktion einer schlaffen Parese, die durch Botulinumtoxin A über eine Hemmung der cholinergen Transmission erfolgt, sollte eine Verminderung des Tonus im UES bzw. im LES erfolgen und damit eine Verbesserung der Dysphagie eintreten. Die Wirksamkeit der Therapie wurde rein subjektiv im Interview erfasst. Aufgrund des retrospektiven Ansatzes der Analyse wurde hier die verspürte Wirkung und keine verspürte Wirkung bzw. Wirkungseintritt und -dauer, sowie Dosierungen der Injektionen beobachtet. Die vorliegende retrospektive Untersuchung belegt die Wirkung von Botulinumtoxin A auf die ösophagealen Sphinkteren nach intramuskulärer Injektion. Von insgesamt 48 Patienten wurden 17 im Bereich des UES und 31 im Bereich des LES therapiert.

\subsubsection{Dysphagien mit Störungen im Bereich des UES (oberer Ösophagussphinkter)}

In der Gruppe UES ( $n=15)$, nach Abzug der beiden Patienten ohne Angaben zur Wirkung, zeigte sich ein Ansprechen auf die intramuskuläre Applikation von Botulinumtoxin A bei $73,3 \%(n=11)$, die sich im Median innerhalb von drei bis fünf Tagen einstellte. Hierbei fiel eine große Spannweite in der Qualität der Wirkung auf. Bei einem Patienten zeigte sich eine geringe Wirkung, so dass er nach der Behandlung zwar in der Lage war den eigenen Speichel zu schlucken, die Ernährung jedoch weiterhin über eine PEG-Sonde erfolgen musste. 81,8\% $(n=9)$ der Patienten mit Wirkung auf Botulinumtoxin $A(n=11)$ gaben hingegen eine komplette Aufhebung der Schluckstörung an. In der Dauer der Wirkung bei einmaliger Injektion ließen sich ebenfalls starke Unterschiede erkennen, die sich in den Angaben der Patienten von einer Woche bis hin zu zwei Jahren zeigten und zu einem Median der Symptombesserung von 3,5 Monaten führten. 
Im Vergleich mit Berichten aus der Literatur konnten die Ergebnisse bestätigt werden. Einige Autoren wiesen eine Symptomreduktion in bis zu 100\% der Patienten nach (Alberty et al. 2000; Blitzer und Brin 1997; Haapaniemi et al. 2001; Moerman et al. 2002; Terre et al. 2008). Murry et al. (2005) konnten nach Erstinjektion einen Erfolg bei $84,6 \%(n=11)$ ihrer Patienten $(n=13)$ verzeichnen, nach Zweitinjektion zeigten allerdings alle Patienten eine Verbesserung der Symptomatik. Bei den ersten Injektionsversuchen mit Botulinumtoxin A im Bereich des UES gelang es Schneider et al. (1994) bei 71,4\% $(n=5)$ der Patienten $(n=7)$ einen Behandlungserfolg zu präsentieren.

Letztere beobachteten einen Eintritt der Wirkung von Botulinumtoxin A auf die Dysphagie innerhalb von etwa acht Tagen. Hingegen berichteten andere Autoren von einem Wirkungseintritt nach drei bis sieben Tagen bzw. ab dem ersten Tag und einer vollen Wirkungsentfaltung nach 15 Tagen (Krause et al. 2008; Quintao Brant et al. 1999).

Von einer sehr variablen Dauer der Botulinumtoxin-A-Wirkung auf die Dysphagie wurde in der Literatur berichtet. So beschrieben Haapaniemi et al. (2001) bei vier beobachteten Patienten eine Symptomlinderung zwischen zwei Wochen und zehn Monaten. Quintao Brant et al. (1999) beobachteten bei 24 Patienten und Murry et al. (2005) bei 13 Patienten ein Benefit der Wirkung bis zu 37 Wochen bzw. einem Jahr. Hingegen berichteten Schneider et al. (1994) von einer maximalen Wirkdauer von fünf Monaten bei sieben betrachteten Patienten.

Qualitative Unterschiede im Ansprechen auf Botulinumtoxin A, wie sie in der vorliegenden Arbeit vorgefunden wurden, sind in der Literatur ebenfalls beschrieben worden. Schneider et al. (1994) berichteten von drei Patienten mit vollständiger Beschwerdefreiheit postoperativ und zwei Patienten mit einer Besserung bei fester Nahrung, aber persitierender Aspiration für Flüssigkeiten. Blitzer und Brin (1997) beschrieben ebenfalls Unterschiede bei den sechs untersuchten Patienten, von einer milden Verbesserung bis zu einer fast normalen Funktion des Schluckens. Diese Beobachtungen wurden subjektiv mittels Interview bei vier Patienten (Moerman et al. 2002) und objektiv bei insgesamt 13 Patienten mit Hilfe der Endoskopie oder der Bariumbrei-Kontrastuntersuchung (Murry et al. 2005) weiter 
bestätigt. Weitere objektive Untersuchungen zeigten Terre et al. (2008). Sie untersuchten 10 Patienten die mit Hilfe der Videofluoroskopie und Manometrie vor und nach der Botulinumtoxin-Injektion. Eine Verbesserung der Öffnung des UES konnte in 89 bis $100 \%$ nachgewiesen werden.

Die Frage nach der Ursache dieser unterschiedlichen Qualitäten konnte bisher nicht abschließend geklärt werden. Alberty et al. (2000) diskutieren hierfür einige mögliche Ursachen. Eine Möglichkeit wäre die Verwendung unterschiedlicher Dosierungen von Botulinumtoxin $\mathrm{A}$. So könnte bei zu geringer Dosis eine unzureichende Chemodenervation des UES resultieren. Allerdings zeigten einige Untersuchungen trotz standardisierten Bedingungen mit gleichen Dosierungen für alle Patienten unterschiedliche klinische Ergebnisse (Alberty et al. 2000; Blitzer und Brin 1997; Moerman et al. 2002). Haapaniemi et al. (2001) zeigten sogar bei einem Patienten eine deutlichere Symptomreduktion bei geringerer Botulinumtoxin-A-Dosis als bei einem anderen Patienten mit höherer Botulinumtoxin-ADosis.

Die Erhöhung der Dosierung von Botulinumtoxin A ist durch die direkte Nachbarschaft des UES zum Larynx und zu Strukturen des Hypopharynx eingeschränkt. Eine Diffusion des Neurotoxins in benachbarte Strukturen ist bei erhöhter Dosierung und vermehrtem Injektionsvolumen wahrscheinlicher. Daher steigen möglicherweise die unerwünschten Wirkungen bei Dosissteigerung an. Beispielsweise wird eine Injektion in den vorderen Anteil des UES bei bestehender Gefahr einer Lähmung des M. cricoarytaenoideus posterior und daraus resultierender Stimmbandparese vermieden (Alberty et al. 2000).

In Betracht kommt daher eine weitere mögliche Ursache: Durch die Diffusion von Botulinumtoxin in benachbarte Strukturen bzw. die falsche Platzierung der Injektionsnadel können andere, für den Schluckakt wichtige Muskeln, gelähmt werden und die Dysphagie würde weiterhin bestehen bleiben. Dies beobachteten Alberty et al. (2000) an einem Patienten, der postoperativ eine vorübergehende Schwäche der hypopharyngealen Boluspropulsion entwickelte. Auch Krause et al. (2008) beschreiben in einem Fallbeispiel eine Diffusion von Botulinumtoxin $A$ in die craniale Pharynxmuskulatur mit anschließender Störung der Pharynxpropulsion. 
Als dritte Option für ein qualitativ unterschiedliches Ansprechen auf die Botulinumtoxin-A-Injektion, wären strukturelle Stenosen des UES aufgrund von persitierenden Hypertrophien oder verändertes Verhältnis von Muskel- und Bindegewebe zu nennen. Vermehrtes Bindegewebe, Entzündungsreaktionen und eine Reduktion des Muskelanteils innerhalb des M. cricopharyngeus fand man in histologischen Präparaten bei Patienten mit unklarer Dysphagie im Bereich des UES (Laurikainen et al. 1992; Zaninotto et al. 2004b). Aufgrund dieser Gegebenheiten könnte es zu unterschiedlichem Diffusionsverhalten der injizierten Lösung und veränderter Plastizität des Gewebes kommen. Weniger Muskelgewebe bedeutet auch weniger Substanz die relaxiert werden könnte, so dass ein geringeres Ansprechen auf Botulinumtoxin A möglich wäre.

Die Vergleichbarkeit der Untersuchungen wird dabei durch die häufig sehr komplexen Krankheitsbilder eingeschränkt. In der vorliegenden Untersuchung kommen beispielsweise oromandibuläre Dystonien und Z. n. cerebralen Ischämien vor. Diese können jedoch neben der inadäquaten Öffnung des UES auch andere wichtige Funktionen des Schluckaktes betreffen. So würde eine alleinige Behandlung des UES nicht zur gewünschten Normalisierung des Schluckaktes führen, wenn nicht gleichzeitig beispielsweise die Zungenbewegung und Larynxfunktion intakt wäre (Alberty et al. 2000).

In der vorliegenden Untersuchung wurden Dosierungen zwischen $30 \mathrm{U}$ und $75 \mathrm{U}$ Botox $^{\circledR}$ bzw. Xeomin ${ }^{\circledR}$, was der Dosierung von Botox ${ }^{\circledR}$ entspricht, injiziert. Der Median der applizierten Dosis liegt hier bei 45 U. Die gewählten Dosierungen fanden sich vergleichbar in der Literatur unter identischer Applikationsmethodik zahlreich wieder. So verwendeten Schneider et al. (1994) zwischen $80 \mathrm{U}$ und $120 \mathrm{U}$ Dysport $^{\circledR}$, das $16 \mathrm{U}$ bis $24 \cup$ Botox $^{\circledR}$ entspricht, Alberty et al. (2000) injizierten hingegen $30 \mathrm{U}$ Botox $^{\circledR}$. $100 \cup$ Botox $^{\circledR}$ wurden in den Untersuchungen von Haapaniemi et al. (2001) und Moerman et al. (2002) verwendet.

Die Höhe der Botulinumtoxin-A-Dosierung ist letztlich nicht ursächlich mit der Wirksamkeit der Therapie assoziiert. Beispielsweise stellten sich zwei der Patienten, die initial nicht auf Botulinumtoxin $A$ ansprachen, noch ein zweites und einer von innen ein drittes Mal vor. Die Dosierung wurde bei einem von $30 \mathrm{U}$ auf $60 \mathrm{U}$ 
verdoppelt und bei dem anderen von $45 \mathrm{U}$ auf $60 \mathrm{U}$ und schließlich auf $75 \mathrm{U}$ erhöht. Trotz der Dosiserhöhung trat keine suffiziente Verbesserung der Dysphagie auf. Eine Fehlplatzierung der Injektionsnadel schloss sich in der vorliegenden Untersuchung aus, da jede Injektion unter visueller Kontrolle erfolgte.

\subsubsection{Dysphagien mit Störungen im Bereich des LES (unterer Ösophagussphinkter)}

Bei 76,7\% ( $n=23)$ der Patienten der Gruppe LES ( $n=30)$, nach Abzug des Patienten ohne Angaben zur Wirkung, konnte in der vorliegenden Untersuchung eine Verbesserung der Dysphagie erreicht werden. Diese stellte sich durchschnittlich innerhalb der ersten beiden Tage post injectionem ein. Wie auch schon bei den Patienten der Gruppe UES dargestellt, zeigte sich hier ebenfalls eine deutliche Variabilität in der Qualität der Wirkung. Ein Patient berichtete von einer Verbesserung der Dysphagie v. a. für flüssige Nahrung. Die Dysphagie für feste Nahrung bestand jedoch weiter. Hingegen berichteten $60,0 \%$ der Patienten $(n=18)$ von einer Wirkung nach Botulinumtoxin-A-Injektion, die zu einer vollständigen Normalisierung des Schluckempfindens führte, d. h. es bestand keinerlei Empfinden einer Dysphagie. In der Dauer der Wirkung bei einmaliger Injektion konnten in dieser Gruppe noch deutlichere Schwankungen von zwei Monaten bis hin zu sechs Jahren beobachtet werden. Im Median lag die Wirkungsdauer bei sechs Monaten.

Diese Befunde aus der vorliegenden Untersuchung stimmen mit Berichten aus der Literatur weitgehend überein. So wurde von einem Behandlungsbenefit von einem Monat post injectionem zwischen $74 \%$ und $93 \%$ berichtet (Annese et al. 1998; Annese et al. 1999; Annese et al. 2000; D'Onofrio et al. 2000; D'Onofrio et al. 2002; Fishman et al. 1996; Martínek et al. 2003; Pasricha et al. 1995; Pasricha et al. 1996 ; Vaezi und Richter 1999). In weiteren Untersuchungen wurde sogar von einer Botulinumtoxinwirkung auf die Dysphagiesymptomatik in 100\% der Fälle berichtet (Annese et al. 1996; Mikaeli et al. 2001). 
Die Botulinumtoxin-Injektionsmethode war dabei in allen oben genannten Untersuchungen gleich. Sie wurde auf dem endoskopischen Weg mit Hilfe einer Sklerosierungsnadel durchgeführt. Auch in der vorliegenden Untersuchung wurde diese Methodik angewandt und gilt als standardisiertes Verfahren (Dunaway und Wong 2001; Mikaeli et al. 2001; Pasricha et al. 1995).

Als Unterschied in den vorliegenden Untersuchungen fand sich allerdings eine variierende Dosierung von Botulinumtoxin A. Diese lag im Bereich zwischen $50 \mathrm{U}$ und $200 \mathrm{U}$ Botox $^{\circledR}$ bzw. bei $200 \mathrm{U}$ Dysport ${ }^{\circledR}$, welches $40 \mathrm{U}$ Botox ${ }^{\circledR}$ entspricht (Annese et al. 1998; Annese et al. 1999; Annese et al. 2000; D`Onofrio et al. 2000; D`Onofrio et al. 2002; Fishman et al. 1996; Martínek et al. 2003; Pasricha et al. 1995; Pasricha et al. 1996 ; Vaezi und Richter 1999).

Interessanterweise stellte die Untersuchung von Annese et al. (2000) dar, dass es im Hinblick auf die initiale Wirkung von Botox ${ }^{\circledR}$, die im Rahmen eines klinischen Symptomenscores gemessen wurde, in Abhängigkeit der Dosierung keine signifikanten Unterschiede gab. Diese prospektiv angelegte Untersuchung verglich drei Gruppen mit unterschiedlichen Dosierungen. Gruppe A wurde $50 \mathrm{U}$ Botox ${ }^{\circledR}$ injiziert, Gruppe B $2 \times 100$ U Botox ${ }^{\circledR}$ und Gruppe C $1 \times 200$ U Botox ${ }^{\circledR}$. Ein signifikanter Unterschied zwischen den Gruppen hinsichtlich eines dosisabhängigen Wirkungseintritts von Botulinumtoxin A konnte nicht nachgewiesen werden.

In der Dauer der Wirkung von Botulinumtoxin A hob sich hingegen Gruppe B signifikant von den anderen beiden Gruppen ab. Diese Gruppe erhielt 2 x $100 \mathrm{U}$ innerhalb von 30 Tagen. In dieser Gruppe zeigte sich 24 Monaten nach der ersten Injektion ein signifikant höherer Anteil an Patienten mit anhaltender Wirkung des Botulinumtoxins im Vergleich zu den anderen beiden Gruppen. Daraus leiteten die Autoren eine weitere Behinderung der nervalen Regeneration bei Zweitinjektion innerhalb von 30 Tagen ab.

In der vorliegenden Untersuchung wurden Dosierungen von Botulinumtoxin A zwischen $20 \mathrm{U}$ und $200 \mathrm{U}$ Botox ${ }^{\circledR}$ verwendet, wobei $200 \mathrm{U}$ erst bei Zweitinjektion verabreicht wurden, wenn der Patient initial nicht auf Botox ${ }^{\circledR}$ reagiert hatte. Daher lag der Median in der Dosierung bei $100 \mathrm{U}$, was sich in der Literatur zahlreich 
bestätigen ließ (Annese et al. 1998; D`Onofrio et al. 2002; Fishman et al. 1996; Kolbasnik et al. 1999; Storr et al. 2002; Zaninotto et al. 2004a).

Die Dauer der Botulinumtoxin-A-Wirkung in der vorliegenden Arbeit war vergleichbar mit Berichten aus der Literatur. Es zeigten 60,9\% ( $n=14)$ der Responder $(n=23)$ eine anhaltende Wirkung nach sechs Monaten. Eine Remissionsrate bei 43\% der Patienten sechs Monate post injectionem beschrieben Gordon und Eaker (1997). Pasricha et al. (1996) schrieben von einer klinischen Besserung bei 55\% der Fälle und Cuillière et al. (1997) von 60\% ihrer Patienten nach sechs Monaten. Weitere Untersuchungen berichteten von einer Symptomlinderung bei $75 \%$ bis 85\% der Patienten, ebenfalls sechs Monate post injectionem (Mikaeli et al. 2001; Wehrmann et al. 1999).

Insgesamt zeigte sich in dieser Analyse eine längere Wirkungsdauer in der Gruppe LES, die im Median bei 6 Monaten lag, im Vergleich zur Gruppe UES, die im Median bei 3,5 Monaten lang. Diese Beobachtung teilen Zaninotto et al. (2004a), die eine mittlere Wirkungsdauer von Botulinumtoxin A von 3 Monaten bei der quergestreiften Muskulatur beobachteten.

\subsection{Vergleich von Respondern und Non-Respondern}

\subsubsection{Gruppe UES (oberer Ösophagussphinkter)}

Im Vergleich des Patientenkollektivs in der Gruppe UES ( $n=15)$, nach Abzug der beiden Patienten ohne Angaben zur Wirkung, von Respondern $(n=11)$ und NonRespondern $(n=4)$ konnten nur wenige Unterschiede beobachtet werden. So waren die Non-Responder durchschnittlich um 9,85 Jahre älter. In der Gruppe der Non-Responder fanden sich viermal so häufig Gewichtsverlust in der Anamnese und eine um 4,3 Jahre längere Beschwerdedauer im Vergleich zu den Respondern. Weder in der Geschlechtsverteilung noch im Hinblick auf Aspirationen in der Vorgeschichte zeigte sich ein Unterschied zwischen den beiden Gruppen. 
In der Literatur fanden sich hierzu nur wenige Angaben. Zaninotto et al. (2004b) verglichen ihr Patientenkollektiv $(n=21)$ nach Verbesserung und keiner Verbesserung der Dysphagie, welches anhand eines klinischen Symptomenscores, Videofluoroskopie und Manometrie ermittelt wurde. Sie konnten keine Unterschiede in Dauer der Beschwerdesymptomatik, der klinischen Ausprägung der Dysphagie, Alter und Geschlecht feststellen. Hingegen zeigten sich signifikante Unterschiede in der pharyngealen Kontraktionsamplitude und in einem Videofluoroskopie-Score post injectionem.

Weitere Untersuchungen beschrieben lediglich das klinische Outcome ihrer Patienten. Die teilweise sehr unterschiedlichen Ergebnisse zeigten einerseits ein vollständiges Ansprechen bei Patienten mit stattgehabter cerebraler Ischämie (Haapaniemi et al. 2001; Murry et al. 2005), andererseits wurde ein klinisches Ansprechen verneint (Alberty et al. 2000). Die unterschiedlichen Ergebnisse könnten aufgrund sehr kleiner Patientenkollektive, die zwischen ein und sechs Patienten beinhalten, zustande gekommen sein und sind daher nur bedingt mit den Ergebnissen der vorliegenden Untersuchung vergleichbar.

\subsubsection{Gruppe LES (unterer Ösophagussphinkter)}

Bei einer Gegenüberstellung von Respondern ( $n=23)$ und Non-Respondern $(n=7)$ des Patientenkollektivs in der Gruppe LES $(n=30)$, nach Abzug des Patienten ohne Angaben zur Wirkung, konnten Unterschiede festgestellt werden. Die Gruppe der Non-Responder war im Durchschnitt 1,5 Jahre älter, litt weniger an Gewichtsverlust und hatte eine um 2,7 Jahre kürzere Beschwerdedauer im Vergleich zu den Respondern. Die kürzere Beschwerdedauer bei den NonRespondern könnte eine mögliche Erklärung für die fehlenden Patienten mit vorherigen Therapieversuchen in der Gruppe der Non-Responder sein. Im Vergleich dazu hatten zwölf Patienten (52\%) in der Gruppe der Responder einen vorherigen Therapieversuch hinter sich. Eine weitere Ursache könnte sich durch das relativ kleine Patientenkollektiv ergeben.

Die unterschiedliche Altersverteilung bei Respondern und Non-Respondern wurde auch in anderen Untersuchungen beobachtet (Annese et al. 2000; Cuillière et al. 
1997; Martínek et al. 2003; Pasricha et al. 1996). So beschrieben Pasricha et al. (1996) und Annese et al. (2000) im Unterschied zur vorliegenden Untersuchung ein signifikant höheres Alter unter Respondern. Im Gegensatz dazu konnten Martínek et al. (2001) und Cuillière et al. (1997) nur eine Tendenz zum höheren Lebensalter unter Respondern im Vergleich zu Non-Respondern feststellen. Ebenso zeigte sich in letzterer Untersuchung eine tendenziell längere Symptomdauer unter den Respondern. Diese Tendenz ließ sich auch in den Untersuchungen von Pasricha et al. (1996) wieder finden. Hingegen wiesen die Responder aus der Untersuchung von Annese et al. (2000) eine um 2,5 Monate kürzere Dauer der Symptomatik im Vergleich mit den Non-Respondern auf.

\subsection{Vergleich von subjektiven und objektiven Ergebnissen}

Im Gesamtkollektiv ( $n=48$ ) der vorliegenden Untersuchung waren $n=25$ Fälle zu finden, in denen ein subjektiver und auch objektiver Vergleich eines Ansprechens bzw. keiner verspürten Wirkung möglich war. Insgesamt ergaben $n=21$ Fälle $(84,0 \%)$ eine Übereinstimmung, in $n=4$ Fällen $(16,0 \%)$ dagegen eine Diskrepanz zwischen Klinik und Diagnostik.

\subsubsection{Gruppe UES (oberer Ösophagussphinkter)}

In der Gruppe UES ( $n=17)$ erhielten vier Patienten eine Videofluoroskopie nach der ersten Injektion. Weitere diagnostische Maßnahmen wurden post injectionem nicht durchgeführt. Hier zeigte sich bei $75,0 \%(n=3)$ eine Übereinstimmung von subjektiven und objektiven Ergebnissen. Bei einem Patienten (25,0\%) widersprachen sich objektives und subjektives Ergebnis.

In einigen Untersuchungen wurde gezeigt, dass keine Rückschlüsse durch die erhobenen objektiven Befunde auf die Klinik gezogen werden dürfen (Crary und Glowasky 1996; Kim et al. 2006). So beschreiben Crary und Glowasky (1996) eine dosisabhängige maximale Öffnung des UES bei einer Botulinumtoxin-Dosierung zwischen 2,5 $\mathrm{U}$ und $25 \mathrm{U}$. Sie konnten eine Diskrepanz zwischen subjektiven 
Äußerungen der Patienten zum Benefit der Therapie und den Ergebnissen der Videofluoroskopie beobachten.

Weiterhin wurden in der Untersuchung von Alberty et al. (2000) bei $n=10$ Patienten subjektive und objektive Ergebnisse miteinander verglichen. Hierbei konnten Patienten in drei verschiedene Gruppen eingeteilt werden. Die erste Gruppe $(n=7)$ zeigte eine deutliche Symptomreduktion in der vom Patienten angegebenen Klinik und objektiv-kontrollierter Videostroboskopie. In der zweiten Gruppe ( $n=2)$ zeigte sich in der Videostroboskopie eine unzureichende Öffnung des UES, die allerdings mit gebesserten Symptomen verbunden war. Die dritte Gruppe $(n=1)$ hingegen zeigte zwar in der Videostroboskopie eine Verbesserung, allerdings blieb klinisch eine Wirkung aus. Alberty et al. (2000) zogen nun einen prognostischen Schluss aus ihren Beobachtungen. Nach ihren Erkenntnissen hätte die erste Gruppe eine gute Prognose für zukünftige Botulinumtoxininjektionen oder cricopharyngeale Myotomien. Die dritte Gruppe hingegen hätte weder Erfolg mit Botulinumtoxin noch mit einer Myotomie. Eine weitere Gruppe, die klinisch keine Symptomreduktion erfuhr, jedoch in der Videostroboskopie eine koordinierte, aber unzureichende Öffnung des UES zeigte, würde nicht von wiederholten Botulinumtoxin-AInjektionen profitieren. Hingegen könnten diese Patienten von einer Myotomie bei strukturellen Stenosen im Bereich des UES Erfolg verspüren.

\subsubsection{Gruppe LES (unterer Ösophagussphinkter)}

In der Gruppe LES ( $n=31$ ) wurden vielfältige diagnostische Mittel herangezogen. Dazu zählten u. a. die wiederholte Ösophagogastroduodenoskopie, Manometrie, CT, Endosonographie oder eine Bariumbrei-Kontrastuntersuchung. Insgesamt konnte in $n=21$ Fällen ein Vergleich gezogen werden. Bei 85,7\% ( $n=18)$ zeigte sich eine Übereinstimmung von subjektiven und objektiven Ergebnissen, eine Diskrepanz fand sich bei $14,3 \%(n=3)$.

Die Ergebnisse waren nahezu deckungsgleich mit zahlreichen Untersuchungen (D`Onofrio et al. 2002; Kolbasnik et al. 1999; Pasricha et al. 1996; Storr et al. 2002). In diesen wurde von einem Zusammenhang in 100\% zwischen der Besserung von klinischen Symptomen und objektiver Diagnostik berichtet. Um eine Ver- 
besserung der Symptomatik zu veranschaulichen, war diese je nach Untersuchung in unterschiedliche Scores eingeteilt worden. Diese Scores beurteilten die Symptomatik nach Dysphagie und Regurgitation. Bei der objektiven Diagnostik kamen Manometrie, der mittlere ösophageale Durchmesser und die ösophageale Retention, die szintigraphisch mit Hilfe einer Technetium-markierten Mahlzeit gemessen wurde, zum Einsatz (D`Onofrio et al. 2002; Kolbasnik et al. 1999; Pasricha et al. 1996; Storr et al. 2002).

\subsection{Vergleich der Therapieverfahren}

Um die Bedeutung von Botulinumtoxin A in der Therapie der Dysphagie herauszustellen, bietet sich der Vergleich mit anderen etablierten Methoden, z. B. Schlucktherapie, Dilatation und Myotomie, an. So konnten Ansprechrate, Wirkungsdauer und Komplikationen miteinander verglichen werden.

\subsubsection{Gruppe UES (oberer Ösophagussphinkter)}

\subsubsection{Ansprechrate und Wirkungsdauer}

In der vorliegenden Untersuchung ließ sich eine Ansprechrate mit 73,3\% feststellen. Dieses Ergebnis deckte sich mit den Ergebnissen vieler Autoren, die von einer Ansprechrate zwischen $71,4 \%$ und $100 \%$ und von einer Wirkungsdauer nach einmaliger Injektion zwischen zwei Wochen und einem Jahr sprachen (Haapaniemi et al 2001; Quintao Brant et al. 1999; Schneider et al. 1994).

Durch die reversible Wirkung von Botulinumtoxin A, stellt diese Therapie eine optimale Möglichkeit bei transienten Dysphagien dar, wie sie in der Akutphase bei Apoplex auftreten kann.

Im Vergleich dazu hatten Shaker et al. (2002) in einer prospektiven, randomisierten Untersuchung über eine Kopf-Elevations-Übung zur Verbesserung des Schluckaktes Verbesserung der Öffnung des UES in 100\% der Fälle beobachtet. Bei dieser Untersuchung wurde diese Übung sechs Wochen lang drei Mal täglich durchgeführt. Die Kontrollgruppe erhielt andere Übungen, die nicht mit einer Ver- 
besserung des Schluckens in Verbindung gebracht wurden. Es ergab sich sowohl in der Videofluoroskopie als auch klinisch in Bezug auf die postdeglutive Aspiration bei der Gruppe mit der Kopf-Elevations-Übung eine signifikante Verbesserung (Shaker et al. 2002).

Im Vergleich zeigte die Dilatation im Bereich des UES eine sehr gute Ansprechrate, die zwischen $90 \%$ und $100 \%$ lag. Diese Daten stammten allerdings aus kleinen Untersuchungen mit Patientenkollektiven zwischen fünf und zehn Patienten. Die Langzeitwirkung wurde zwischen einer Woche und 27 Monaten, im Median mit 21 Monaten angegeben. Eine andere Untersuchung konnte eine Langzeitansprechrate von 55,7\% im Zeitraum von 12-18 Monaten darstellen (Hatlebakk et al. 1998; Mathieu et al. 1997; Solt et al. 2001; Wang et al. 2005).

Die Myotomie zeigte hingegen eine Ansprechrate von $60 \%$ bis $80 \%$ (Ellis et al. 1996; Mason et al. 1998; Poirier et al. 1997; Steele et al. 2007). Es wurde ein Langzeitansprechen bei Patienten in $50 \%$ bis $73 \%$ der Fälle nach fünf bis 17 Monaten beschrieben (Lebo et al. 1976; Zaninotto et al. 2004b).

\subsubsection{Komplikationen}

Die Botulinumtoxininjektion in den oberen Ösophagussphinkter stellt eine nebenwirkungsarme Methode dar. Es wurden keine systemischen Nebenwirkungen beobachtet, lediglich eine Patientin berichtete von einer "hängenden Lippe“ und einem "Singultus“. Darüber hinaus könnte diese Nebenwirkung ebenso von Botulinumtoxininjektionen im Bereich des Mundbodens und der Speicheldrüsen resultieren, welche zeitgleich erfolgten. Weitere Komplikationen aufgrund der teilweise schweren und mehrfachen Begleiterkrankungen im Patientenkollektiv zeigten sich nicht. Dabei litten immerhin 76,5\% ( $n=13)$ der Patienten der Gruppe UES $(n=17)$ an weiteren Erkrankungen, wovon ein Großteil in den Bereich der kardiovaskulären Belastung fiel. In anderen Untersuchungen zur Therapie mit Botulinumtoxin im Bereich des UES wurden ebenfalls keine systemischen Nebenwirkungen beobachtet, wobei lokale Nebenwirkungen im Sinne einer vorübergehenden, postprandialen Regurgitation oder Aspirationspneumonie zu 0\% bis $20 \%$ auftraten (Ashan et al. 2000; Crary und Glowasky 1996; Zaninotto et al. 2004b). Aufgrund 
einer Vollnarkose mit notwendiger Intubation stellte die transorale Botulinumtoxininjektion, trotz der kurzen Interventionszeit von 10-30 Minuten, ein relativ risikoreiches Verfahren dar. Auch die perkutanen Methoden bargen trotz der lokalen Anästhesie durch den recht langen Weg der Injektionsnadel ein gewisses $\mathrm{Maß}$ an Risiko.

Bei der Dilatation wurden Nebenwirkungsraten von $0 \%$ bis $20 \%$ beobachtet. Diese wurden als Pneumothorax, beispielsweise verursacht durch die Fehlsondierung des Drahtes, über den der Ballonkatheter platziert wird, und oberflächliche Schleimhautverletzung beschrieben (Solt et al. 2001; Steele et al. 2007).

Die Myotomie hingegen stellte aufgrund einer notwendigen Vollnarkose und postoperativer stationärer Betreuung eine hohe Invasivität dar. Die Risiken lagen in Infektionen, v. a. der gefürchteten Mediastinitis, Nervenverletzungen und Fistelbildungen. Die postoperative Mortalität wurde mit $0 \%$ bis $20 \%$ angegeben (Loizou et al. 1980; Zaninotto et al. 2004b).

Konservative Therapieoptionen, zu denen die professionelle Schlucktherapie mit Mendelsohn-Manöver gezählt wird, zeigten hingegen weder Nebenwirkungen, noch war innen eine Invasivität nachzuweisen (Walther 1998).

\subsubsection{Botulinumtoxininjektion als Diagnostikum}

Die Bedeutung von Botulinumtoxin als Diagnostikum wurde von mehreren Autoren beschrieben (Blitzer und Brin 1997; Alberty et al. 2000; Ashan et al. 2000). Die Wirkung von intramuskulär injiziertem Botulinumtoxin ist der einer operativen Myotomie ähnlich, daher auch der Begriff der "chemischen“ Myotomie in Bezug auf Botulinumtoxin. Eine erfolgreiche Botulinumtoxintherapie wurde daher als positiver prognostischer Faktor für das Ansprechen einer operativen Myotomie angesehen. So könnten Patienten mit einer chronischen Dysphagie nach erfolgreicher Botulinumtoxininjektion einer operativen Myotomie zugeführt werden, um die wiederholten Injektionen zu umgehen (Alberty et al. 2000; Ashan et al. 2000; Blitzer und Brin 1997). 


\subsubsection{Gruppe LES (unterer Ösophagussphinkter)}

\subsubsection{Ansprechrate und Wirkungsdauer}

Die Botulinumtoxininjektion in den LES zeigte einen Therapieerfolg bei 76,7\% der Patienten mit einer medianen Wirkdauer von sechs Monaten. Diese Angaben konnten in der Literatur weiter bestätigt werden. Injektionen mit Botulinumtoxin A in den LES zeigten eine mediane Wirkungsdauer von sechs Monaten bei einer initialen Ansprechrate von 74\% bis 93\% (Annese et al. 1999; Martínek et al. 2003; Vaezi und Richter 1999). Aufgrund der nachlassenden Wirkung waren dabei wiederholte Injektionen notwendig, um einen nachhaltigen Erfolg zu erreichen. Annese et al. (1998) konnten bei wiederholten Applikationen von Botulinumtoxin eine mediane Langzeitwirkung von $75 \%$ innerhalb von einem medianen Beobachtungszeitraum von 24 Monaten nachweisen.

Die pneumatische Dilatation zeigte ebenfalls eine klinische Besserung von bis zu $90 \%$. Eine Rezidivfreiheit wurde mit über fünf Jahren bei $40 \%$ der Fälle beschrieben (Eckardt et al. 2004) bzw. blieben 67\% der Fälle in einem Beobachtungszeitraum von zwei bis sieben Jahren rezidivfrei (Lake und Wong 2006).

Die Myotomie nach Gottstein-Heller zeigte eine Verbesserung der Symptomatik in über $90 \%$. Mit einer anhaltenden Symptomfreiheit nach fünf Jahren war in über $80 \%$ der Fälle zu rechnen. Rezidive hingegen waren nach sechs bis neun Jahren in 8-10\% der Fälle nachweisbar (Csendes et al. 1988; Herbella et al. 2008; Mineo und Ambrogi 2004). In der Untersuchung von Tatum et al. (2007) wurde gezeigt, dass die Patienten $(n=56)$ nach einer Myotomie nach Gottstein-Heller $(n=16)$ sowohl im Vergleich mit der pneumatischen Dilatation als auch der Botulinumtoxininjektion ( $\mathrm{n}=10$ für beide zusammen) eine signifikant höhere Besserung der subjektiven und objektiven Dysphagie hatten. Die Dysphagie wurde anhand eines Fragebogens und der Manometrie gemessen. Dabei wurden der Wirkungseintritt und die Wirkungsdauer nicht näher betrachtet.

Medikamentöse Therapieoptionen mit Nifedipin und Isosorbiddinitrat zeigten sehr variable Ergebnisse. Deren Ansprechrate lag zwischen 50\% und 90\%. Durch die 
Entwicklung einer Toleranz gegenüber der Wirkung von Nifedipin bzw. Isosorbiddinitrat waren in der Regel befriedigende Langzeiterfolge bei dieser Therapieoption begrenzt (Lake und Wong 2006). In der vorliegenden Untersuchung wurden zuvor $n=13$ Patienten $(41,9 \%)$ medikamentös behandelt. Sie beschrieben eine mangelhafte Wirkung im weiteren Verlauf und entschieden sich daher zur Behandlung mit Botulinumtoxin A.

\subsubsection{Komplikationen und Risiken}

Bei der Komplikationsrate zeigte sich die Therapie mit Botulinumtoxin als sichere Therapieoption. Neben leichten retrosternalen Schmerzen, die sich als dosisunabhängig erwiesen, und Sodbrennen, traten keine weiteren unerwünschten Wirkungen auf. Diese unerwünschten Wirkungen von Botulinumtoxin A waren selten und wurden nur vorübergehend beobachtet. In der vorliegenden Analyse betrug die Rate an unerwünschten Arzneimittelwirkungen 9,7\%. Im Vergleich zu anderen Therapieoptionen war die Therapie mit Botulinumtoxin A am wenigsten invasiv. Durch eine leichte Sedierung wurde das Risiko einer Vollnarkose vermieden. Das Verwenden einer handelsüblichen Sklerosierungsnadel stellte darüber hinaus eine sichere Injektionsmethode dar. Durch die lokale Injektion waren systemische Nebenwirkungen unwahrscheinlich und wurden in der vorliegenden Untersuchung nicht beobachtet. Eine ambulante Behandlung mit Botulinumtoxin $A$ auch bei multimorbiden und älteren Patienten war daher möglic und relativ sicher anwendbar.

In der vorliegenden Untersuchung waren mehrere ältere und multimorbidere Patienten eingeschlossen worden. Der Altersmedian lag bei 57 Jahren, die ältesten Patienten hatten jedoch ein Alter bis 85 Jahren. Zudem litten 71,0\% ( $n=22)$ der Patienten an Begleiterkrankungen, wobei kardiovaskuläre Erkrankungen den größten Anteil ausmachten. Diese zählen zu den häufigsten Ursachen für perioperative Morbidität und Letalität (Zwißler 2008). Daneben zählen pulmonale Vorerkrankungen $\mathrm{zu}$ den häufigsten Ursachen postoperativer Intervention (Zollinger et al. 2008). Neben den Begleiterkrankungen standen 83,9\% $(n=26)$ der Patienten unter Dauermedikation. Auch hier zeigte sich eine Tendenz in der Medikation, wie sie beispielsweise bei Herzinsuffizienz verabreicht wird: ACEHemmer, B-Rezeptorenblocker, Diuretika, Calcium-Kanalblocker, Nitrate/Nitrite, 
Digitalispräparate (Herold 2004). Der Vorteil der Botulinumtoxininjektion bei Achalasie war die Vermeidung des Risikos einer Vollnarkose, die bei einer Myotomie notwendig wäre bzw. der Perforation bei pneumatischer Dilatation. Im Kollektiv der vorliegenden Untersuchung konnten keine Komplikationen während und nach der Botulinumtoxininjektion beobachtet werden.

Bei der pneumatischen Dilatation wurde durch eine leichte Sedierung ebenfalls das Risiko einer Vollnarkose vermieden. Eine der Hauptkomplikationen bei der pneumatischen Dilatation stellte jedoch die Perforation dar, die in 1-3\% der Behandlungen auftrat (Lake und Wong 2006). Darüber hinaus bestand das Risiko eine Refluxerkrankung in 4-16\% zu entwickeln (Vela et al. 2004; Vela et al. 2006). In der vorliegenden Analyse hatten $35,5 \%(n=11)$ der Patienten $(n=31)$ den Versuch einer pneumatischen Dilatation hinter sich. Keiner berichtete von einer Perforation, jedoch beschrieb ein Patient (9,1\%) keinen Wirkungseintritt. 90,9\% dieser Patienten $(n=10)$ verspürten einen Progress der Achalasie und wünschten eine andere Therapieoption. Bei der medikamentösen Therapie hingegen traten in bis zu $30 \%$ unerwünschte Arzneimittelwirkungen wie Hypotension, Kopfschmerzen und periphere Ödeme auf (Gelfond et al. 1982). Da die medikamentöse Therapie eine geringe Invasivität aufweißt, ist sie v. a. bei beginnender Achalasie und zur Überbrückung bei Wartezeiten auf andere Therapien geeignet. Aufgrund der Risiken, die ein operatives Vorgehen mit sich bringt, stellt die Myotomie die höchste Invasivität dar. Die operativen Komplikationen, die Schleimhautläsionen, Perforation und postoperative Leckage miteinbeziehen, wurden in der Literatur allerdings mit unter 10\% angegeben (Falkenback et al. 2003; Patti et al. 1999; Richards et al. 2004).

\subsubsection{Kombination von verschiedenen Therapien}

In der Gruppe LES ( $n=31)$ hatten 41,9\% $(n=13)$ der Patienten bereits Therapieversuche hinter sich bzw. wurden 35,5\% ( $n=11)$ weiterhin mit medikamentöser Therapie, wie Nitrate und Calciumantagonisten, behandelt. So war bei $35,5 \%(n=11)$ eine pneumatische Dilatation durchgeführt worden. Jeweils $3,2 \%(n=1)$ hatten eine Botulinumtoxin-A-Injektion bzw. Myotomie hinter sich. Wurde das Ansprechen auf eine Botulinumtoxininjektion in Beziehung zu vorhergehenden Therapien gesetzt, 
so zeigte sich, dass sowohl Patienten mit vorhergehender pneumatischer Dilatation $(n=11)$ von $90,9 \%(n=10)$ als auch Patienten mit erfolgter Myotomie bzw. Botulinumtoxininjektion zu 100\% $(n=1)$ einen positiven Effekt mit Botulinumtoxin $A$ verspürten.

In der Literatur wurde ebenfalls die Kombination verschiedener Therapien untersucht. Es zeigte sich, dass sich weder eine vorherige Therapie mit Botulinumtoxin, oder pneumatischer Dilatation noch eine Myotomie auf die Wirkung einer Botulinumtoxininjektion auswirkt (Storr et al. 2002). Dagegen führte eine vorherige Botulinumtoxininjektion sowohl bei der pneumatischen Dilatation, als auch bei der Myotomie zu einer erhöhten Komplikationsrate (Horgan und Pellegrini 1999; Srinivasan et al. 2000). 


\subsection{Schlussfolgerung}

Die vorliegende Untersuchung zeigte, dass die intramuskuläre Injektion von Botulinumtoxin A in den Ösophagus bei etwa 75,6\% $(n=34)$ der Patienten $(n=45)$, die unter Dysphagie leiden, eine Symptombesserung bewirkte. Durchschnittlich hielt diese Wirkung drei bis sechs Monate an, bevor sich die Patienten zu einer Reinjektion wieder vorstellten. Diese Wirkung zeigte sich im Wesentlichen unabhängig von der Hauptdiagnose und der Geschlechtsverteilung.

Durch die Blockierung der cholinergen Transmission zeigte Botulinumtoxin einen relaxierenden Effekt an der Muskulatur. Dieser Effekt zeigte einen deutlichen therapeutischen Benefit bei der Dysphagie aufgrund einer Motilitätsstörung im Bereich sowohl des UES als auch des LES. Auch wenn die Zahl der NonResponder in dieser Analyse 24,4\% ( $n=11)$ betrug, stellt die Injektionsmethode mit Botulinumtoxin A für die Behandlung der Dysphagie eine sehr gute Alternative wie auch eine gute Ergänzung zu den anderen therapeutischen Maßnahmen dar.

Die Anwendungsgebiete von Botox ${ }^{\circledR}$ sind aktuell auf Indikationen außerhalb des Gastrointestinaltrakts beschränkt, wie z. B. Blepharospasmus, hemifazialem Spasmus und koexistierenden lokalen Dystonien, Torticollis spasmodicus, dynamische Spitzfußstellung infolge Spastizität bei infantiler Zerebralparese, fokaler Spastizität der Hand bei erwachsenen Schlaganfallpatienten und primärer Hyperhidrosis axillaris (Rote Liste 2009). Daher stellt die Therapie der Dysphagie mit Botulinumtoxin A einen „off-label-use“ dar. Durch zahlreiche Untersuchungen und eigene Erfahrungen konnte bei Motilitätsstörungen im Bereich der beiden Ösophagussphinkteren verbunden mit dem Leitsymptom Dysphagie eine positive Wirkung von Botulinumtoxin Typ A nachgewiesen werden. 


\section{$5 \quad$ ZUSAMMENFASSUNG}

Dysphagien finden sich sowohl in der Hals-Nasen-Ohrenheilkunde als auch in der Gastroenterologie. Insbesondere bei Achalasie und Nussknackerösophagus, aber auch postoperativ nach laryngopharyngealen Tumoren und Dystonien im orofazialen Bereich zeigt sich die Symptomatik der Dysphagie. Diese kann effektiv durch eine intramuskuläre Injektion von Botulinumtoxin A behandelt werden.

Das Patientenkollektiv der vorliegenden retrospektiven Analyse bestand aus 48 Patienten mit Dysphagie unterschiedlicher Genese. Aufgrund einer Dysfunktion des UES konnten 17 Patienten im Mittel mit $45 \mathrm{U}$ in diesem Bereich und infolge einer Achalasie 31 Patienten im Mittel mit $100 \mathrm{U}$ Botulinumtoxin A im Bereich des LES behandelt werden. Bei $75,6 \%$ der Patienten $(n=45)$ konnte eine Verbesserung der Symptomatik innerhalb von 0-8 Tagen, im Mittel innerhalb der ersten beiden postinterventionellen Tage, nachgewiesen werden. Nach drei bis sechs Monaten stellten sich die Patienten, aufgrund eines Nachlassens der Wirkung, wieder vor. Aufgrund zahlreicher Begleiterkrankungen und Dauermedikamente zeigte sich ein morbides Patientenkollektiv, das v. a. an Herzkreislauferkrankungen litt. Im Vergleich zwischen Respondern und Non-Respondern zeigten sich keine wesentlichen Unterschiede in der Geschlechtsverteilung. Hingegen konnte eine Tendenz zu einem höheren Lebensalter in der Gruppe der Non-Responder festgestellt werden.

Das hier angewendete Verfahren hat sich im Vergleich mit anderen Therapieoptionen als einfach und komplikationsarm erwiesen. Schwere und dauerhafte unerwünschte Wirkungen fehlen gänzlich. So stellt Botulinumtoxin A v. a. bei morbideren und älteren Patienten eine gute Therapieoption dar. Durch die reversible Wirkung an der motorischen Endplatte können ebenso transiente Dysphagien effektiv behandelt werden. Auch chronische Dysphagien eignen sich hervorragend bei wiederholten Anwendungen zur Therapie mit Botox ${ }^{\circledR}$. Die Wirkung von Botox ${ }^{\circledR}$ bei Dysphagie wird bereits seit 1994 beobachtet und konnte seitdem mehrfach nachgewiesen werden. Die vorliegenden Ergebnisse bestätigen den therapeutischen Nutzen einer intramuskulären Injektion von Botulinumtoxin A zur Behandlung der Dysphagie. 
6 ANHANG:

\section{Einverständniserklärung und Behandlungsbogen}

\section{I Einverständniserklärung UES}

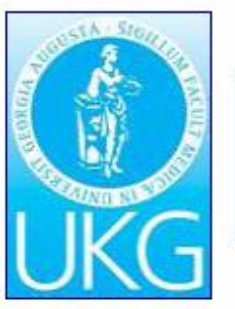

\section{Universitäts-HNO-Klinik Göttingen Botulinum-Toxin-Sprechstunde}

\section{Einverständniserklärung (Aufklärung)}

zur Botulinum-Toxin-Injektion bei Dysphagie:

Mit umseitiger Unterschrift bestätige ich, dass ich diese Erklärung gelesen und verstanden habe, dass ich die Möglichkeit hatte, mit dem Arzt deren Inhalt ausführlich und zu meiner Zufriedenheit zu besprechen, und dass ich mit dem beschriebenen Vorgehen einverstanden bin.

Die Unterschrift dieser Einverständniserklärung bedingt für mich keinerlei Verpflichtung, und ich darf ohne irgendwelche Verbindlichkeiten entscheiden, dass ich an dieser Behandlung nicht weiter teilnehmen möchte.

Wenn Nahrungsmittel, die einen Anteil von Botulinum-Toxin enthalten, gegessen werden, können sie eine Nahrungsmittelvergiftung (Botulismus) verursachen. Wird diese Substanz jedoch in der angewendeten extrem kleinen Menge in das betroffene Schleimhautareal gespritzt, verursacht sie eine vorübergehende Unterbrechung der Nervenübertragung auf die betroffenen Muskeln.

Ich wurde darüber informiert, dass dieses Präparat auf dem Markt für andere Indikationen als die hier durchgeführte Indikation zugelassen ist (Off-label-use) und an der Göttinger Universitäts-HNO-Klinik seit 1990 Patienten, die unter Muskelzuckungen im Gesicht leiden, mit gleicher Methode behandelt werden. Systemische Nebenwirkungen (Auswirkungen auf den gesamten Körper, außerhalb der behandelten Region) sind nie aufgetreten.

Ich wurde weiterhin darüber informiert, dass diese Behandlung nicht das grundsätzliche Problem der überflüssigen Versorgung der Muskeln mit Nervenimpulsen löst, sondern es lediglich zu einer vorübergehenden Unterbrechung der Nervenübertragung kommt.

Nach bisherigen Erfahrungen tritt dieser Effekt nach etwa 3 Tagen ein und bleibt voraussichtlich über die Dauer von 3-4 Monaten bestehen. Nach dieser Zeit ist die erneute Injektion von Botulinum-Toxin zur Beseitigung der Symptome notwendig.

\section{Nebenwirkungen:}

Ich wurde über die Risiken, die mit dieser Behandlung verbunden sind, aufgeklärt. Insbesondere kann es zu einer vorübergehenden Schwächung der Stimme (Dysarthrie/Dysphonie), Luftnot, Aspiration, Lungenentzündung (Pneumonie), Blutungen und Schluckbeschwerden kommen. Wie bei Injektionen, die an anderen Stellen durchgeführt werden, können ein leichter Bluterguß und ein leichtes Unwohlsein gelegentlich auftreten. Die Behandlung kann das Legen einer Magensonde notwendig machen. Sie kann in örtlicher Betäubung oder in Vollnarkose durchgeführt werden. Mir wurde erklärt, dass diese Risiken ärztlich vertretbar sind. 
Fortsetzung der Einverständniserklärung zur Botulinum-Toxin-Injektion bei spasmodischer Dysphonie

Die Fragen nach generalisierten Muskelerkrankungen (z.B. Myasthenia gravis), derzeitiger Aminoglykosid-Antibiotika-Therapie (z.B. Streptomycin, Neomycin, Gentamicin) oder bestehender Schwangerschaft/Stillzeit habe ich verneint.

Herr Prof. Dr. med. R. Laskawi und/oder werden eine minimale Dosis von Botulinum-Toxin in das betroffene Areal spritzen.

Ich werde mich bemühen, allen Anweisungen zu folgen und das Vorgehen zu erleichtern und ich werde unter ärztlicher Überwachung nach der Injektion bleiben, bis mir mitgeteilt wird, dass eine derartige sorgfältige Beobachtung nicht mehr notwendig ist.

Aufgrund dieser Informationen erkläre ich mich damit einverstanden, dass die vorgenannte Behandlung einschließlich der dafür notwendigen ärztlichen Untersuchungen an mir durchgeführt wird, wobei ich mir vorbehalte, meine Einwilligung jederzeit widerrufen zu können. Ferner erkläre ich meine Bereitschaft, mich an die ärztlichen Anordnungen für die Zeit vor, während und nach der Behandlung zu halten.

Meine persönlichen Daten und meine Krankengeschichte unterliegen den üblichen Bestimmungen über die ärztliche Schweigepflicht. Ich bin damit einverstanden, dass Photographien, auf denen ich erkennbar bin sowie die Beschreibung meiner Krankengeschichte in medizinischen Zeitschriften, Büchern und anderen Publikationen oder Medien veröffentlicht werden oder auf wissenschaftlichen Fachtagungen vorgetragen werden.

Göttingen, den 
VI. II Behandlungsbogen UES

\section{Universitäts-HNO-Klinik Göttingen Botulinum-Toxin-Behandlungsbogen \\ Dysphagie, M. cricopharyngeus}

Aufkleber

Injektions-Punkte:

Gesamtdosis:

rechts lateral

links lateral

dorsal
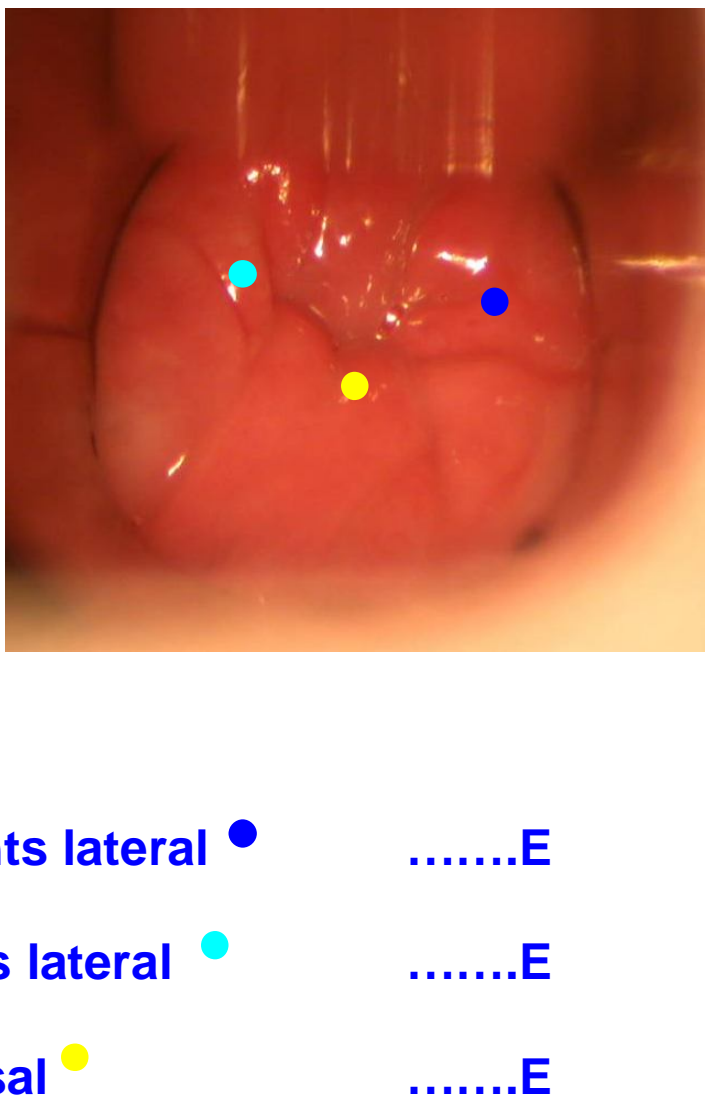

$E$

\section{E} E

\section{Datum:}

Unterschrift:
Präparat:

Einheiten 


\section{LITERATURVERZEICHNIS}

Alberty J, Oelerich M, Ludwig K, Hartmann S, Stoll W (2000): Efficacy of botulinum toxin A for treatment of upper esophageal sphincter dysfunction. Laryngoscope $\underline{110}(7), 1151-1156$

Annese V, Basciani M, Perri F, Lombardi G, Frusciante V, Simone P, Andriulli A, Vantrappen $G$ (1996): Controlled trial of botulinum toxin injection versus placebo and pneumatic dilation in achalasia. Gastroenterology 111(6), 1418-1424

Annese V, Basciani M, Borrelli O, Leandro G, Simone P, Andriulli A (1998): Intrasphincteric injection of botulinum toxin is effective in long-term treatment of esophageal achalasia. Muscle Nerve 21(11), 1540-1542

Annese V, Bassotti G, Coccia G, D'Onofrio V, Gatto G, Repici A, Andriulli A (1999): Comparison of two different formulations of botulinum toxin A for the treatment of oesophageal achalasia. GISMAD Achalasia Study Group. Aliment Pharmacol Ther $\underline{13}(10), 1347-1350$

Annese V, Bassotti G, Coccia G, Dinelli M, D'Onofrio V, Gatto G, Leandro G, Repici A, Testoni PA, Andriulli A (2000): A multicentre randomised study of intrasphincteric botulinum toxin in patients with oesophageal achalasia. GISMAD Achalasia Study Group. Gut $\underline{46}(5), 597-600$

Arnon SS, Schechter R, Inglesby TV, Henderson DA, Bartlett JG, Ascher MS, Eitzen E, Fine AD, Hauer J, Layton M (2001): Botulinum toxin as a biological weapon: medical and public health management. JAMA 285(8), 1059-1070

Ashan SF, Meleca RJ, Dworkin JP (2000): Botulinum toxin injection of the cricopharyngeus muscle for the treatment of dysphagia. Otolaryngol Head Neck Surg 122(5), 691-695

Binscheck T, Wellhörner HH: Struktur und Wirkmechanismen der BotulinumNeurotoxine; in: Botulinumtoxin-Therapie im Kopf-Hals-Bereich, 2. aktualisierte und erweiterte Auflage, herausgegeben von Laskawi, R, Roggenkämper, P; Urban\&Vogel-Verlag, München 2004: S. 25-45

Blitzer A, Brin MF (1997): Use of botulinum toxin for diagnosis and management of cricopharyngeal achalasia. Otolaryngol Head Neck Surg 116(3), 328-330 
Böhme G: Sprach-, Sprech-, Stimm- und Schluckstörungen, Band 1, 4. Auflage; Urban\&Fischer-Verlag, Müchen 2003

Bortolotti M, Mari C, Lopilato C, Porrazzo G, Miglioli M (2000): Effects of sildenafil on esophageal motility of patients with idiopathic achalasia. Gastroenterology $\underline{118}(2), 253-257$

Breit S, Heckmann M (2000): Botulinumtoxin. Hautarzt 51(11), 874-891

Bueno R, Swanson SJ, Jaklitsch MT, Lukanich JM, Mentzer SJ, Sugarbaker DJ (2001): Combined antegrade and retrograde dilation: a new endoscopic technique in the management of complex esophageal obstruction. Gastrointest Endosc 54(3), 368-372

Crary MA, Glowasky AL (1996): Using botulinum toxin A to improve speech and swallowing function following total laryngectomy. Arch Otolaryngol Head Neck Surg 122(7), 760-763

Csendes A, Braghetto I, Mascaró J, Henríquez A (1988): Late subjective and objective evaluation of the results of esophagomyotomy in 100 patients with achalasia of the esophagus. Surgery 104(3), 469-475

Csendes A, Braghetto I, Burdiles P, Korn O, Csendes P, Henríquez A (2006): Very late results of esophagomyotomy for patients with achalasia: clinical, endoscopic, histologic, manometric, and acid reflux studies in 67 patients for a mean follow-up of 190 months. Ann Surg 243(2), 196-203

Cuillière C, Ducrotté P, Zerbib F, Metman EH, de Looze D, Guillemot F, Hudziak H, Lamouliatte H, Grimaud JC, Ropert A (1997): Achalasia: outcome of patients treated with intrasphincteric injection of botulinum toxin. Gut 41 (1), 87-92

Dantas RO, Cook IJ, Dodds WJ, Kern MK, Lang IM, Brasseur JG (1990): Biomechanics of cricopharyngeal bars. Gastroenterology 99(5), 1269-1274

Da Silveira EB, Rogers AI (2002): Achalasia: a review of therapeutic options and outcomes. Compr Ther 28(1), 15-22

Di Nardo G, Blandizzi C, Volta U, Colucci R, Stanghellini V, Barbara G, Del Tacca M, Tonini M, Corinaldesi R, De Giorgio R (2008): Review article: molecular, 
pathological and therapeutic features of human enteric neuropathies. Aliment Pharmacol Ther 28(1), 25-42

Donner MW, Bosma JF, Robertson DL (1985): Anatomy and physiology of the pharynx. Gastrointest Radiol 10 (3), 196-212

D'Onofrio V, Annese V, Miletto P, Leandro G, Marasco A, Sodano P, laquinto G (2000): Long-term follow-up of achalasic patients treated with botulinum toxin. Dis Esophagus 13(2), 96-101

D'Onofrio V, Miletto P, Leandro G, laquinto G (2002): Long-term follow-up of achalasia patients treated with botulinum toxin. Dig Liver Dis $\underline{34}(2), 105-110$

Dunne J, Hayes M, Cameron D (1993): Botulinum toxin A for cricopharyngeal dystonia. Lancet $\underline{342(8870),} 559$

Dunaway PM, Wong RK (2001): Achalasia. Curr Treat Options Gastroenterol 4(1): 89-100

Eckardt VF, Gockel I, Bernhard G (2004): Pneumatic dilation for achalasia: late results of a prospective follow up investigation. Gut $\underline{53}(5), 629-633$

Ekberg O (1987): Dysfunction of the pharyngo-oesophageal segment in patients with normal opening of the upper oesophageal sphincter: a cineradiographic study. Br J Radiol 60(715), 637-644

Ellies M, Laskawi R, Rohrbach-Volland S, Arglebe C, Beuche W (2002): Botulinum toxin to reduce saliva flow: selected indications for ultrasound-guided toxin application into salivary glands. Laryngoscope $\underline{112(1), 82-86}$

Ellis FH Jr, Gibb SP, Williamson WA (1996): Current status of cricopharyngeal myotomy for cervical esophageal dysphagia. Eur J Cardiothorac Surg 10(12), 1033-1038

Falkenback D, Johansson J, Oberg S, Kjellin A, Wenner J, Zilling T, Johnsson F, Von Holstein CS, Walther B (2003): Heller's esophagomyotomy with or without a 360 degrees floppy Nissen fundoplication for achalasia. Long-term results from a prospective randomized study. Dis Esophagus 16(4), 284-290 
Fishman VM, Parkman HP, Schiano TD, Hills C, Dabezies MA, Cohen S, Fisher RS, Miller LS (1996): Symptomatic improvement in achalasia after botulinum toxin injection of the lower esophageal sphincter. Am J Gastroenterol 91(9), 1724-1730

Frick H, Leonhardt H, Starck D: Spezielle Anatomie II - Eingeweide, Nervensystem, Systematik Muskeln und Leitungsbahnen. 4. Auflage; Georg Thieme Verlag, Stuttgart 1992

Friedenberg F, Gollamudi S, Parkman HP (2004): The use of botulinum toxin for the treatment of gastrointestinal motility disorders. Dig Dis Sci $\underline{49}(2), 165-175$

Gelfond M, Rozen P, Gilat T (1982): Isosorbide dinitrate and nifedipine treatment of achalasia: a clinical, manometric and radionuclide evaluation. Gastroenterology $\underline{83}(5), 963-969$

Gockel I, Eckardt VF, Schmitt T, Junginger T (2005): Pseudoachalasia: a case series and analysis of the literature. Scand J Gastroenterol 40(4), 378-85

Gordon JM, Eaker EY (1997): Prospective study of esophageal botulinum toxin injection in high-risk achalasia patients. Am J Gastroenterol 92(10), 1812-1817

Goyal RK, Chaudhury A (2008): Physiology of normal esophageal physiology. J Clin Gastroenterol $\underline{42(5), 610-619}$

Greger R: Membranpotential, übersetzt und bearbeitet von H. und S. Silbernagel; in: Lehrbuch der Physiologie, 2. Auflage, herausgegeben von Klinke, $R$ und Silbernagel, S; Georg Thieme-Verlag Stuttgart 2000, S. 47-58

Grübel C, Borovicka J, Schwizer W, Fox M, Hebbard G (2008): Diffuse esophageal spasm. Am J Gastroenterol 103(2), 450-457

Haapaniemi JJ, Laurikainen EA, Pulkkinen J and Marttila RJ (2001): Botulinum toxin in the treatment of cricopharyngeal dysphagia. Dysphagia $\underline{16}(3), 171-175$

Hatlebakk JG, Castell JA, Spiegel J, Paoletti V, Katz PO Castell DO (1998): Dilatation therapy for dysphagia in patients with upper esophageal sphincter dysfunction-manometric and symptomatic response. Dis Esophagus 11(4), 254259 
Hayashi T, McMahon H, Yamasaki S, Binz T, Hata Y, Südhof TC Niemann H (1994): Synaptic vesicle membrane fusion complex: action of clostridial neurotoxins on assembly. EMBO J $\underline{13}(21), 5051-5061$

Herbella FA, Aquino JL, Stefani-Nakano S, Artifon EL, Sakai P, Crema E, Andreollo NA, Lopes LR, De Castro Pochini C, Corsi PR (2008): Treatment of achalasia: lessons learned with Chagas' disease. Dis Esophagus 21(5),461-467

Herold G: Innere Medizin. Eigenverlag, Köln 2004

Horgan S, Pellegrini CA (1999): Botulinum toxin injections for achalasia sym-

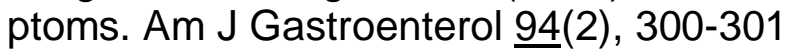

Hornbostel H: Innere Medizin in Praxis und Klinik in 4 Bänden, Band IV: Verdauungstrakt, Ernährungsstörungen, Stoffwechsel, Vergiftungen. 3. Auflage; Georg Thieme Verlag, Stuttgart 1986

Kasar PA, Khadilkar VV, Tibrewala VN (2007): Allgrove Syndrome. Indian J Pediatr $\underline{74}(10)$, 959-961

Kim DY, Park Cl, Ohn SH, Moon JY, Chang WH, Park SW (2006): Botulinum toxin type A for poststroke cricopharyngeal muscle dysfunction. Arch Phys Med Rehabil $\underline{87}(10), 1346-1351$

Kolbasnik J, Waterfall WE, Fachnie B, Chen Y, Tougas G (1999): Long-term efficacy of Botulinum toxin in classical achalasia: a prospective study. Am J Gastroenterol 94(12), 3434-3439

Kraichely RE, Farrugia G (2006): Achalasia: physiology and etiopathogenesis. Dis Esophagus 19(4), 213-223

Krause E, Schirra J, Gürkov R (2008): Botulinum toxin a treatment of cricopharyngeal dysphagia after subarachnoid hemorrhage. Dysphagia 23(4), 406-10

Kreyden OP, Geiges ML, Böni R, Burg G (2000): Botulinumtoxin: Vom Gift zum Medikament. Ein historischer Rückblick. Hautarzt 51(10), 733-737

Lake JM, Wong RK (2006): Review article: the management of achalasia - a comparison of different treatment modalities. Aliment Pharmacol Ther 24(6), 909918 
Laskawi R (1998): Die therapeutische Anwendung von Botulinum-Toxin im HalsNasen-Ohren-Bereich, Teil 2. Bewegungsstörungen der kraniozervikalen Muskulatur (außer mimischer Mukulatur) sowie Störungen des autonomen Nervensystems. HNO $\underline{46}(4), 366-373$

Laskawi R (2008): The use of botulinum toxin in head and face medicine: an interdisciplinary field. Head Face Med 10, 4:5

Laskawi R, Ellies M (2007): The role of botulinum toxin in the management of head and neck cancer patients. Curr Opin Otolaryngol Head Neck Surg 15(2), 112-116

Laurikainen E, Aitasalo K, Halonen P, Falck B, Kalimo H (1992): Muscle pathology in idiopathic cricopharyngeal dysphagia. Enzyme histochemical and electron microscopic findings. Eur Arch Otorhinolaryngol 249(4), 216-223

Lebo CP, U KS, Norris FH Jr (1976): Cricopharyngeal myotomy in amyotrophic lateral sclerosis. Laryngoscope $\underline{86}(6), 862-868$

Lew RJ, Shah JN, Chalian A, Weber RS, Williams NN, Kochman ML (2004): Technique of endoscopic retrograde puncture and dilatation of total esophageal stenosis in patients with radiation-induced strictures. Head Neck $\underline{26}(2), 179-183$

Loizou LA, Small M, Dalton GA (1980): Cricopharyngeal myotomy in motor neurone disease. J Neurol Neurosurg Psychiatry $\underline{43}(1), 42-45$

Martínek J, Siroký M, Plottová Z, Bures J, Hep A, Spicák J (2003): Treatment of patients with achalasia with botulinum toxin: a multicenter prospective cohort study. Dis Esophagus 16(3), 204-209

Mason RJ, Bremner CG, DeMeester TR, Crookes PF, Peters JH, Hagen JA, DeMeester SR (1998): Pharyngeal swallowing disorders: selection for and outcome after myotomy. Ann Surg 228(4), 598-608

Mathieu J, Lapointe G, Brassard A, Tremblay C, Brais B, Rouleau GA, Bouchard JP (1997): A pilot study on upper esophageal sphincter dilatation for the treatment of dysphagia in patients with oculopharyngeal muscular dystrophy. Neuromuscul Disord $\underline{7}(1), 100-104$ 
Mikaeli J, Fazel A, Montazeri G, Yaghoobi M, Malekzadeh R (2001): Randomized controlled trial comparing botulinum toxin injection to pneumatic dilatation for the treatment of achalasia. Aliment Pharmacol Ther 15(9), 1389-1396

Mineo TC, Ambrogi V (2004): Long-term results and quality of life after surgery for oesophageal achalasia: one surgeon's experience. Eur J Cardiothorac Surg 25(6), 1089-1096

Moerman M (2006): Cricopharyngeal Botox injection: indications and technique. Curr Opin Otolaryngol Head Neck Surg 14(6), 431-436

Moerman M, Callier Y, Dick C, Vermeersch H (2002): Botulinum toxin for dysphagia due to cricopharyngeal dysfunction. Eur Arch Otorhinolaryngol 259(1), 1-3

Müller C, Lorenz J (2005): Dysphagie. Pneumologe 2: 367-378

Müller M: Chirurgie - Für Studium und Praxis. 7. Auflage; Medizinische Verlagsund Informationsdienste, Breisach 2004

Murry T, Wasserman T, Carrau RL, Castillo B (2005): Injection of botulinum toxin A for the treatment of dysfunction of the upper esophageal sphincter. Am $J$ Otolaryngol $\underline{26}(3), 157-162$

Netter FH: Atlas der Anatomie des Menschen, 2. Auflage; Georg Thieme Verlag Stuttgart 2000

Nüßgens Z: Historische Entwicklung: Vom Toxin zum therapeutischen Ansatz; in: Botulinumtoxin-Therapie im Kopf-Hals-Bereich, 2. Auflage, herausgegeben von Laskawi, R und Roggenkämper, P; Urban\&Vogel-Verlag München 2004, S.15-19

Palmer JB, Drennan JC, Baba M (2000): Evaluation and treatment of swallowing impairments. Am Fam Physician 61(8), 2453-2462

Park W, Vaezi MF (2005): Etiology and pathogenesis of achalasia: the current understanding. Am J Gastroenterol 100(6), 1404-1414

Pasricha PJ, Ravich WJ, Hendrix TR, Sostre S, Jones B, Kalloo AN (1994): Treatment of achalasia with intrasphincteric injection of botulinum toxin. A pilot trial. Ann Intern Med 121(8), 590-591 
Pasricha PJ, Ravich WJ, Hendrix TR, Sostre S, Jones B, Kalloo AN (1995): Intrasphincteric botulinum toxin for the treatment of achalasia. $\mathrm{N}$ Engl $\mathrm{J}$ Med 332(12), 774-778

Pasricha PJ, Rai R, Ravich WJ, Hendrix TR, Kalloo AN (1996): Botulinum toxin for achalasia: long-term outcome and predictors of response. Gastroenterology $\underline{110}(5), 1410-1415$

Patti MG, Pellegrini CA, Horgan S, Arcerito M, Omelanczuk P, Tamburini A, Diener U, Eubanks TR, Way LW (1999): Minimally invasive surgery for achalasia: an 8-year experience with 168 patients. Ann Surg 230(4), 587-593

Pehlivanov N, Pasricha PJ (2006): Achalasia: botox, dilatation or laparoscopic surgery in 2006. Neurogastroenterol Motil 18(9), 799-804

Pohl D, Tutuian R (2007): Achalasia: an overview of diagnosis and treatment. J Gastrointestin Liver Dis 16(3), 297-303

Poirier NC, Bonavina L, Taillefer R, Nosadini A, Peracchia A, Duranceau A (1997): Cricopharyngeal myotomy for neurogenic oropharyngeal dysphagia. $J$ Thorac Cardiovasc Surg 113(2), 233-240

Pototschnig C, Thumfart W: Dysphagie in: Botulinumtoxin-Therapie im Kopf-HalsBereich, 2. Auflage, herausgegeben von Laskawi, R, Roggenkämper, $P$ und andere; Urban\&Vogel-Verlag München 2004, S.185-195

Prakash C, Freedland KE, Chan MF, Clouse RE (1999): Botulinum toxin injections for achalasia symptoms can approximate the short term efficacy of a single pneumatic dilation: a survival analysis approach. Am J Gastroenterol 94(2), 328333

Pschyrembel: Pschyrembel Klinisches Wörterbuch, 261. Auflage, unter der Leitung von Helmut Hildebrandt; Walter de Gruyter-Verlag, Berlin 2007

Pütz A: Empfehlungen zum Umgang mit der Substanz Botulinumtoxin, in: Botulinumtoxin-Therapie im Kopf-Hals-Bereich, 2. Auflage, herausgegeben von Laskawi, R und Roggenkämper, $\mathrm{P}$ unter Mitarbeit namenhafter Autoren; Urban\&Vogel-Verlag München 2004, S. 46-54 
Quintao Brant CQ, Siqueira ES, Ferrari AP Jr (1999): Botulinum toxin for oropharyngeal dysphagia: case report of flexible endoscope-guided injection. Dis Esophagus 12(1), 68-73

Quirk D, Friedman L: Chapter 47: Approach to Gastrointestinal Problems in the Elderly in: Textbook of Gastroenterology, 3. Auflage, herausgegeben von Yamada, T und Mitarbeiter; Lippincott Williams \& Wilkins-Verlag Philadelphia 1999, S. 1015-1033

Rebecchi F, Giaccone C, Farinella E, Campaci R, Morino M (2008): Randomized controlled trial of laparoscopic Heller myotomy plus Dor fundoplication versus Nissen fundoplication for achalasia - long-term results. Ann Surg 248(6), 10231030

Renz-Polster H, Braun J: Basislehrbuch Innere Medizin, 2. Auflage; Urban \& Fischer Verlag München 2001

Restivo DA, Marchese-Ragona R, Staffieri A, De Grandis D (2000): Successful botulinum toxin treatment of dysphagia in oculopharyngeal muscular dystrophy. Gastroenterology $\underline{119}(5), 1416$

Restivo DA, Palmeri A, Marchese-Ragona R (2002): Botulinum toxin for cricopharyngeal dysfunction in Parkinson's disease. N Engl J Med 346(15), 1174-1175

Richards WO, Torquati A, Holzman MD, Khaitan L, Byrne D, Lutfi R, Sharp KW (2004): Heller myotomy versus Heller myotomy with Dor fundoplication for achalasia: a prospective randomized double-blind clinical trial. Ann Surg 240(3), 405-412

Rote Liste: Arzneimittelverzeichnis für Deutschland (einschließlich EUZulassungen und bestimmter Medizinprodukte) herausgegeben und verlegt von Rote Liste® Service GmbH Frankfurt a.M. 2009

Schneider I, Thumfart WF, Pototschnig C, Eckel HE (1994): Treatment of dysfunction of the cricopharyngeal muscle with botulinum $A$ toxin: introduction of a new, noninvasive method. Ann Otol Rhinol Laryngol 103(1), 31-35

Schwörer H: Achalasie; in: Botulinumtoxin-Therapie im Kopf-Hals-Bereich, 2. Auflage, herausgegeben von Laskawi, R und Roggenkämper, P; Urban\&VogelVerlag München 2004, S. 196-202 
Scott AB (1980): Botulinum toxin injection into extraocular muscles as an alternative to strabismus surgery. J Pediatr Ophthalmol Strabismus 17(1), 21-25

Shaker R, Easterling C, Kern M, Nitschke T, Massey B, Daniels S, Grande B, Kazandjian M, Dikeman K (2002): Rehabilitation of swallowing by exercise in tubefed patients with pharyngeal dysphagia secondary to abnormal UES opening. Gastroenterology 122(5), 1314-1321

Singh S, Hamdy S (2005): The upper oesophageal sphincter. Neurogastroenterol Motil $\underline{17}(1), 3-12$

Solt J, Bajor J, Moizs M, Grexa E, Horváth PO (2001): Primary cricopharyngeal dysfunction: treatment with balloon catheter dilatation. Gastrointest Endosc $\underline{54}(6)$, 767-771

Srinivasan R, Vela MF, Tutuian R, Katz PO, Castell DO (2000): Prior botulinum toxin injection may compromise outcome of pneumatic dilatation in achalasia. Am J Gastroenterol 95(9), 2436-2437 (Abstract)

Steele NP, Tokayer A, Smith RV (2007): Retrograde endoscopic balloon dilation of chemotherapy- and radiation-induced esophageal stenosis under direct visualization. Am J Otolaryngol 28 (2), 98-102

Stendal C: Practical guide to gastrointestinal function testing. First edition; Blackwell Science Oxford 1997

Storr $M$, Born $P$, Frimberger $E$, Weigert $N$, Rösch $T$, Meining $A$, Classen $M$, Allescher HD (2002): Treatment of achalasia: the short-term response to botulinum toxin injection seems to be independent of any kind of pretreatment. BMC Gastroenterol 2(19), 1-8

Tatum RP, Wong JA, Figueredo EJ, Martin V, Oelschlager BK (2007): Return of esophageal function after treatment for achalasia as determined by impedancemanometry. J Gastrointest Surg 11(11), 1403-1409

Terre R, Valles M, Panades A, Mearin F (2008): Long-lasting effect of a single botulinum toxin injection in the treatment of oropharyngeal dysphagia secondary to upper esophageal sphincter dysfunction: a pilot study. Scand J Gastroenterol $\underline{43}(11), 1296-1303$ 
Vaezi MF, Richter JE (1999): Diagnosis and management of achalasia. American College of Gastroenterology Practice Parameter Committee. Am J Gastroenterol $\underline{94}(12), 3406-3412$

Vela MF, Richter JE, Khandwala F, Blackstone EH, Wachsberger D, Baker ME, Rice TW (2006): The long-term efficacy of pneumatic dilatation and Heller myotomy for the treatment of achalasia. Clin Gastroenterol Hepatol 4(5), 580-587

Vela MF, Richter JE, Wachsberger D, Connor J, Rice TW (2004): Complexities of managing achalasia at a tertiary referral center: use of pneumatic dilatation, Heller myotomy and botulinum toxin injection. Am J Gastroenterol 99(6), 1029-1036

Walther EK (1998): Schluckstörungen. HNO $\underline{46}(8), 712-728$

Wang AY, Kadkade R, Kahrilas PJ, Hirano I (2005): Effectiveness of esophageal dilation for symptomatic cricopharyngeal bar. Gastrointest Endosc $\underline{61}(1), 148-152$

Wehrmann T, Kokabpick H, Jacobi V, Seifert H, Lembcke B, Caspary WF (1999): Long-term results of endoscopic injection of botulinum toxin in elderly achalasic patients with tortuous megaesophagus or epiphrenic diverticulum. Endoscopy 31(5), 352-358

Young JA, Cook DI, Lingard JM, Van Lennep EW, Wegman W: Funktion des Magen-Darm-Trakts übersetzt und bearbeitet von $H$. und S. Silbernagel, in: Lehrbuch der Physiologie, 2. Auflage, herausgegeben von Klinke, R und Silbernagel, S unter Mitarbeit namenhafter Autoren; Georg Thieme-Verlag Stuttgart 2000, S. $387-433$

Zaninotto G, Annese V, Costantini M, Del Genio A, Costantino M, Epifani M, Gatto G, D'Onofrio V, Benini L, Contini S (2004a): Randomized controlled trial of botulinum toxin versus laparoscopic heller myotomy for esophageal achalasia. Ann Surg 239(3), 364-370

Zaninotto G, Marchese Ragona R, Briani C, Costantini M, Rizzetto C, Portale G, Zanetti L, Masiero S, Costantino M, Nicoletti L (2004b): The role of botulinum toxin injection and upper esophageal sphincter myotomy in treating oropharyngeal dysphagia. J Gastrointest Surg $\underline{8}(8)$, 997-1006

Zollinger A, Hofer C, Kuhnle G, Schauer A, Schwender D, Mann R: Anästhesie bei Patienten mit Lungenerkrankungen, in: Die Anästhesiologie, 2. Auflage, heraus- 
gegeben von Rossaint R, Werner C, Zwißler B; Springer Medizin Verlag Heidelberg 2008, S. 1268-1285

Zwißler B: Anästhesie bei Erkrankungen von Herz und Kreislauf, in: Die Anästhesiologie, 2. Auflage, herausgegeben von Rossaint R, Werner C, Zwißler B; Springer Medizin Verlag Heidelberg 2008, S. 1212-1265 


\section{DANKSAGUNG}

Abschließend möchte ich mich bei allen herzlich bedanken, die zum Gelingen dieser Arbeit beigetragen haben. Ganz besonders bei:

- Prof. Dr. med. Laskawi für die freundliche Überlassung des Themas, die interessante Weiterbildung innerhalb der Botox-Sprechstunde, die sehr gute Betreuung und die schnelle Korrektur und Durchsicht des Manuskripts mit vielen wichtigen Anregungen.

- Prof. Dr. med. Schwörer für die gute interdisziplinäre Zusammenarbeit, die sehr gute Betreuung, die zeitnahen und überaus hilfreichen Korrekturen und sein Engagement, z. B. bei der Überlassung der endoskopischen Fotografien.

- Schwester Christiane Pietzka von der Endoskopie für die freundliche Zusammenarbeit und inr persönliches Engagement, zahlreiche Patientendaten, Fotografien und Bilder zu sammeln.

- dem Team der Botox-Sprechstunde für die freundliche Aufnahme und die lehrreichen Stunden. 


\section{LEBENSLAUF}

Am 02. Januar 1981 bin ich als erstes Kind von Peter Bartsch und Rosemarie Bartsch, geborene Weisser, in Villingen-Schwenningen geboren. In St. Georgen im Schwarzwald wuchs ich auf und besuchte dort von August 1987 bis Juli 1991 die Grundschule am Ruppertsberg. Im September 1991 wechselte ich auf das Gymnasium St. Georgen, wo ich im Juni 2000 meine Hochschulreife erlangte.

Nach einem 2-monatigen Krankenpflegepraktikum am Hubertuskrankenhaus Berlin, welches ich im August und September 2000 absolvierte, begann ich ein 6monatiges Praktikum in der gynäkologischen Praxis Dr. med. B. Schembra in St. Georgen.

Im April 2001 begann ich das Studium der Humanmedizin an der Georg-AugustUniversität Göttingen, wo ich im März 2003 meine Ärztliche Vorprüfung und im März 2004 die 1. Ärztliche Prüfung absolvierte.

Mit dem Praktischen Jahr begann ich im Februar 2006. Mein 1. Tertial verbrachte ich in der Pädiatrie am Städtischen Klinikum Wolfsburg. Das 2. Tertial führte ich in der Inneren Medizin im Regionalspital Laufenburg, Schweiz, und das 3. Tertial in der Allgemeinchirurgie im Good Health Hospital Wanganui, Neuseeland, durch.

Im November 2007 absolvierte ich die 2. Ärztliche Prüfung an der Georg-AugustUniversität Göttingen.

Nach Erlangen der Approbation bin ich seit dem 01.01.2008 in der Pädiatrie des Städtischen Klinikums Wolfsburg als Assistenzärztin beschäftigt und verfolge das Ziel des Facharztes für Pädiatrie. 